\title{
Predicting physiological aging rates from a range of quantitative traits using machine learning
}

\author{
Eric D. Sun ${ }^{1}$, Yong Qian ${ }^{1}$, Richard Oppong ${ }^{1}$, Thomas J. Butler ${ }^{1}$, Jesse Zhao ${ }^{1}$, Brian H. Chen ${ }^{2}$, \\ Toshiko Tanaka ${ }^{1}$, Jian Kang ${ }^{3}$, Carlo Sidore ${ }^{4}$, Francesco Cucca ${ }^{4}$, Stefania Bandinelli ${ }^{5}$, Gonçalo R. \\ Abecasis $^{3}$, Myriam Gorospe ${ }^{6}$, Luigi Ferrucci ${ }^{1}$, David Schlessinger $^{6}$, Ilya Goldberg7, Jun Ding ${ }^{1}$ \\ ${ }^{1}$ Longitudinal Studies Section, Translational Gerontology Branch, National Institute on Aging, Baltimore, MD \\ 21224, USA \\ ${ }^{2}$ Department of Epidemiology, The Herbert Wertheim School of Public Health and Human Longevity Science, UC \\ San Diego, La Jolla, CA 92093, USA \\ ${ }^{3}$ Department of Biostatistics, University of Michigan, Ann Arbor, MI 48109, USA \\ ${ }^{4}$ Istituto di Ricerca Genetica e Biomedica, Consiglio Nazionale delle Ricerche, Monserrato, Italy \\ ${ }^{5}$ Geriatric Unit, Azienda Sanitaria di Firenze, Florence, Italy \\ ${ }^{6}$ Laboratory of Genetics and Genomics, National Institute on Aging, Baltimore, MD 21224, USA \\ ${ }^{7}$ ViQi, Inc., Santa Barbara, CA 93111, USA
}

Correspondence to: Luigi Ferrucci, David Schlessinger, llya Goldberg, Jun Ding; email: ferruccilu@grc.nia.nih.gov, schlessingerd@grc.nia.nih.gov, ilya@viqi.org, jun.ding@nih.gov

Keywords: physiological aging rate, quantitative trait, machine learning, aging clock, mortality, personalized medicine Received: February 18, $2021 \quad$ Accepted: September 29, $2021 \quad$ Published: October 29, 2021

Copyright: (C) 2021 Sun et al. This is an open access article distributed under the terms of the Creative Commons Attribution License (CC BY 3.0), which permits unrestricted use, distribution, and reproduction in any medium, provided the original author and source are credited.

\section{ABSTRACT}

It is widely thought that individuals age at different rates. A method that measures "physiological age" or physiological aging rate independent of chronological age could therefore help elucidate mechanisms of aging and inform an individual's risk of morbidity and mortality. Here we present machine learning frameworks for inferring individual physiological age from a broad range of biochemical and physiological traits including blood phenotypes (e.g., high-density lipoprotein), cardiovascular functions (e.g., pulse wave velocity) and psychological traits (e.g., neuroticism) as main groups in two population cohorts SardiNIA ( 6,100 participants) and InCHIANTI ( 1,400 participants). The inferred physiological age was highly correlated with chronological age $\left(R^{2}>0.8\right)$. We further defined an individual's physiological aging rate (PAR) as the ratio of the predicted physiological age to the chronological age. Notably, PAR was a significant predictor of survival, indicating an effect of aging rate on mortality. Our trait-based PAR was correlated with DNA methylation-based epigenetic aging score $(r=0.6)$, suggesting that both scores capture a common aging process. PAR was also substantially heritable $\left(h^{2} 0.3\right)$, and a subsequent genome-wide association study of PAR identified significant associations with two genetic loci, one of which is implicated in telomerase activity. Our findings support PAR as a proxy for an underlying whole-body aging mechanism. PAR may thus be useful to evaluate the efficacy of treatments that target aging-related deficits and controllable epidemiological factors.

\section{INTRODUCTION}

The study of possible intrinsic aging rates is itself venerable and continues to be motivated by Peter Medawar's pioneering question: does aging occur by a fundamental process independent of-though affected by - overt disease? [1]. To answer this question, the investigation of human aging rates must confront the experimental hurdle presented by our long lifespans. As a result, longitudinal studies of aging in human cohorts 
typically require significant resources invested over many years. A reproducible framework for estimating physiological age as a measurement of intrinsic age progression would permit the analysis and evaluation of treatments that target aging-related debilitation without a need for expensive long-term studies [2-4]. To be useful, biological aging rate measurements should be relatively stable across time in longitudinal studies $[3,5$, 6], account for aging at different biological levels [7-9], and ideally be associated with disease risk and mortality $[3,8]$. Early attempts to estimate "biological age" using individual biomarkers of aging (e.g., telomere length, pulse wave velocity, grip strength, etc) produced only modest correlations with chronological age [10-17]. Since there is little variation in human age at natural death [18], true biological age is unlikely to deviate markedly from chronological age- - hence the need for a more tightly correlated measure of biological age.

Measurements of biological age based on DNA methylation patterns have provided significantly higher correlation $\left(\mathrm{R}^{2}>0.8\right)$ with chronological age $[19,20]$ and can also be associated with disease and death [2127]. However, the role of DNA methylation in the aging process is still largely unclear [28]; it does not account for higher biological levels such as physiology and phenotype, which are presumably affected by other aging-related mechanisms.

Given that aging is a complex process that manifests in virtually all tissues and organs [29-31], it seems likely that composite indices that summarize many molecular and physiological traits to estimate biological age may provide a route to greater understanding [5, 9]. Many efforts have turned to quantitative models for measuring biological age using sets of biomarkers; these models have had success in measuring variations associated with physiological decline, but may require certain assumptions about biological aging or carefully curated sets of traits [32-39]. An increasingly popular approach for robust measurement of biological age is machine learning, which involves building mathematical models on a set of training data to predict a target variable [40]. Machine learning has been used to predict biological age from DNA methylation patterns [19, 20], blood serum markers [41], image data [42-44], and transcriptomic and proteomic signatures [45-47]. Despite these advances, few biological age measurements integrate traits from distinct hierarchical levels (e.g., proteome, metabolome, and phenome) and across the multiple organ systems in which aging is manifest.

Here we use a machine learning approach with a broad range of biochemical and physiological traits including blood phenotypes (e.g., high-density lipoprotein), cardiovascular functions (e.g., pulse wave velocity) and psychological traits (e.g., neuroticism) as main groups from the SardiNIA longitudinal study of aging [48, 49] to estimate human physiological age, a metric for phenotypic and functional age progression [7]. From the estimated physiological age, we derive a physiological aging rate (PAR) as a measure of individual age progression relative to chronological age. This framework is used to determine the most ageresponsive, well-correlated, and relevant traits with respect to physiological aging. Independently, we develop a model for estimating physiological age in the InCHIANTI longitudinal study [50, 51], for which we find results similar to those in the SardiNIA study and show positive correlation between PAR and the epigenetic aging rate (EAR) based on DNA methylation. We determine that PAR is a significant predictor of survival and is appreciably heritable. Additionally, a genome-wide association study (GWAS) reveals single-nucleotide polymorphisms (SNPs) that are significantly associated with PAR. These findings may help to elucidate the physiological and genetic underpinnings of the human aging rate.

\section{MATERIALS AND METHODS}

Our primary goal was to predict an individual's physiological aging rate (PAR) based on his/her quantitative traits by using machine learning methods. We investigated several machine learning models, trait selection and dimensionality reduction methods, and data cleaning and sampling strategies to develop an optimized framework. Each machine learning model was trained on the entire trait set using chronological age as the target variable. The predictive frameworks were evaluated using the coefficient of determination $\left(\mathrm{R}^{2}\right)$ between the predicted age and chronological age. The most promising model was then used to generate physiological ages for individuals in SardiNIA and separately trained to generate physiological ages for individuals in InCHIANTI.

The general pipeline for estimating individual physiological aging rate is listed below (also see Supplementary Figure 1; we discuss the details in each sub-section of Materials and Methods):

1. The datasets were cleaned to remove missing values. The strategy for data cleaning was heuristically determined to preserve the maximum number of traits and samples (see Supplementary Materials).

2. Individuals were binned by chronological age (see Supplementary Figure 2 and Supplementary Materials for details). 
3. Samples were split randomly into the training $(90 \%)$ and testing (10\%) sets in each of the chronological age bins.

4. Traits were selected by the Fisher score computed on the training set and trait values were transformed with linear discriminant analysis. The number of traits selected was predetermined as the number of Fisher-rank-ordered traits corresponding to the maximum average $\mathrm{R}^{2}$ value between predicted and actual age across 10,000 training-testing splits.

5. Machine learning models were trained to predict chronological age on the training data set and validated using the testing data set.

6. Steps 3-5 were reiterated 10,000 times to create enough training-testing splits to ensure wide coverage of all participants. The physiological age of an individual was the mean predicted age across these 10,000 training-testing splits.

7. The physiological aging rate (PAR) was calculated as the ratio between physiological age and chronological age for a given individual.

For the SardiNIA study, after computing PARs, we estimated the heritability of PAR from pedigree information. We also treated PAR as a quantitative trait to perform a genome-wide association study (GWAS) and to identify genetic variants (i.e., single nucleotide polymorphisms, SNPs) significantly associated with PAR. We examined the reproducibility of PAR measurements across time using the follow-up studies from each dataset. We determined significant differences in PAR measurements between deceased and living subjects. For the InCHIANTI study, we compared our PARs with corresponding DNA methylation age measurements [19, 20].

\section{Datasets}

\section{SardiNIA longitudinal study}

Funded by the National Institute on Aging in 2001, the SardiNIA Project (age range 14.0 to 101.3 years, with a mean of 43.7 years; $57 \%$ female) is a longitudinal study of human aging on the island of Sardinia, which is notable for its long-lived population [48, 49]. Several characteristics made this population ideal for modeling physiological age. First, the study cohort is relatively homogenous, which reduced genetic noise. Second, environmental factors are more controlled in an island population, which reduced possible noise due to environmental heterogeneity. Third, the SardiNIA dataset included sequencing information for most of its cohort, making the study suitable for GWAS [52]. The size of sequence coverage and availability of family relationships permitted heritability estimates from pedigree information. The longitudinal nature of the SardiNIA study (i.e., three follow-up waves in addition to the baseline study) allowed us to study individual aging rate dynamics across time [52].

The dataset included over 6100 participants and more than 200 physiological and cognitive traits in total. After cleaning missing entries in a trait-blind, iterative manner, we retained over 4000 subjects and roughly 140 traits with complete data; they were used for model development.

\section{InCHIANTI longitudinal study}

The InCHIANTI study was designed to understand the physiological factors that affect mobility and included measurements from many different physiological systems [50, 51]. The studied population spanned two sites: Greve in Chianti (11,709 inhabitants >65 years) and Bagno a Ripoli (Village of Antella, 4704 inhabitants $>65$ years). The study started in 1998 with 1,463 subjects (age range 21.0 to 102.0 years, with a mean of 69.0 years; $56 \%$ female) and included four follow-up waves with the fourth wave retaining 687 subjects. The majority $(1,020)$ of the subjects were elderly (>65 years old). The study included approximately 2,500 trait variants spanning six main physiological subsystems: central nervous system, peripheral nervous system, perceptual system, muscles, bones/joints, and energy production/delivery [51]. The InCHIANTI study provided similar advantages as the SardiNIA study in genetic and environmental homogeneity and its longitudinal nature. In addition, InCHIANTI included data on DNA methylation for many subjects, which were used to calculate DNA methylation age estimates [50].

For the SardiNIA and InCHIANTI cohorts, we chose to apply the machine learning models to the full set of traits of each cohort. The main reason for this choice is that the vast majority of current machine learning methods require that individuals have all trait values available (i.e., no missing data), and consequently, if we were to try to use the same traits for both cohorts, we could use only the smaller list of overlapping traits. Another advantage of the current approach is that the same method can be applied to data from any new study without requiring that it provides exactly the same set of traits.

\section{Machine learning algorithms}

We explored both classification and regression models including the random forest classifier (RFC) [53], elastic net (ElNet) regression [54], k-nearest neighbors regressor (kNNR) [55], LASSO regression [56], 
multiple linear regression, and support vector machine (SVM) [57] (see Supplementary Materials for model specifications). Classification models required bin labels, which were computed as the center chronological age of each bin. Regression algorithms utilized the individual chronological ages but required binning to uniformly sample subjects from the age bins. The machine learning algorithms were adapted from the open-source Scikit-Learn machine learning library [58].

\section{Random forest classifier}

The random forest classifier from the Scikit-Learn library [58] was selected for its optimal predictive performance. Our model used 30 estimators, the Gini impurity criterion, minimum sample splits of 2 , and minimum sample leaf sizes of 1 . These model parameter choices were determined heuristically. The primary advantage afforded by the random forest model is its natural ability to include ordinal and binary features that are present in both SardiNIA and InCHIANTI studies (e.g., yes/no answers to questionnaires, categorized qualitative ratings of high, moderate, and low, etc).

\section{Trait selection and dimensionality reduction}

\section{Fisher trait scoring and selection}

We utilized the Fisher scoring method for trait selection [59]. For the training data, the trait values and the corresponding chronological age bin labels were used to assign a Fisher score to each trait. The number of Fisher rank-ordered traits that maximized the average $\mathrm{R}^{2}$ between predicted and actual ages over 10,000 trainingtesting splits was used for model training. After trait selection, the RFC model included 79 traits while the common-trait RFC model included 52 traits in the baseline SardiNIA study. Further dimensionality reduction was performed using linear discriminant analysis (LDA), which slightly improved the predictive accuracy of the models (see Supplementary Materials, Supplementary Figure 3).

\section{Linear discriminant analysis}

Linear Discriminant Analysis (LDA) was used for dimensionality reduction before machine learning. LDA is a generalization of the Fisher discriminant and transforms a set of traits into $\mathrm{N}-1$ linear discriminants where $\mathrm{N}$ is the number of discrete classes (i.e., number of age bins). We implemented the default "LinearDiscriminantAnalysis" function from the ScikitLearn library [58].

\section{Statistical metrics}

To measure the predictive performance of the different predictive models, we employed several statistical metrics to determine the accuracy of model-predicted physiological age with respect to chronological age.

\section{Pearson correlation (r) and coefficient of determination $\left(R^{2}\right)$}

The primary measurement of correlation was the Pearson correlation $r$ and the closely related coefficient of determination $\mathrm{R}^{2}$, which represented the proportion of the variance in the target variable that could be explained by the traits used in the model. The $\mathrm{R}^{2}$ value was computed as the square of the Pearson correlation coefficient $r,\left(R^{2}=r^{2}\right)$ :

\section{Mean absolute error (MAE)}

The mean absolute error represented the magnitude of difference between two continuous variables. The MAE was calculated for $N$ measurements of two variables $(\mathrm{x}, \mathrm{y})$ as:

$$
M A E=\frac{\sum_{i=1}^{N}\left|x_{i}-y_{i}\right|}{N}
$$

The MAE measured the average difference between chronological age and physiological age in a cohort of $N$ individuals and is represented in units of years.

\section{Measurements of age progression}

\section{Physiological aging rate}

The physiological aging rate (PAR) represented the characteristic aging rate for an individual from birth to the time of measurement and was computed as the ratio of predicted physiological age to chronological age:

$$
P A R=\frac{\text { Physiological Age }}{\text { Chronological Age }}
$$

We chose to use the ratio rather than the difference between physiological and chronological age because the ratio accounts for the age of an individual such that the same difference in ages would result in a larger deviation in the PAR of a younger subject than that of an older subject.

\section{Interpretation of PAR}

When PAR $<1$, the individual's physiological age is less than their chronological age, they are aging slower than expected for their age cohort; when PAR > 1, the individual's physiological age is greater than their chronological age, so they are aging faster than expected.

\section{Testing the association between PAR and mortality}

We used two methods to study the effect of PAR on survival (or mortality) using the SardiNIA cohort in which we were able to collect information on mortality. We recorded 263 deaths among the 4,415 SardiNIA participants that had their PAR estimated using all 
traits. The deceased participants had ages from 16.3 to 76.7 years. We first applied the widely used survival analysis model (Cox proportional hazards model) to test whether PAR is a predictor for survival while adjusting for age and sex. The PAR measurement was base-2 logarithm transformed [i.e., $\log _{2}(\mathrm{PAR})$ ] such that the effect size of PAR on survival could be conveniently interpreted as the hazard ratio with a two-fold change of PAR. Second, we performed a comparative analysis between the 263 deceased participants and the remaining participants on their aging rates. To remove the potential confounding of chronological age, we randomly paired an age-matched living participant to each deceased participant. The differences in the PAR measurements of the pairs were then calculated as $\triangle \mathrm{PAR}=\mathrm{PAR}_{\text {deceased }}-$ $\mathrm{PAR}_{\text {living, }}$, and a corresponding $p$-value was obtained from a one-sided $(\triangle \mathrm{PAR}>0)$ one-sample $t$-test. The agematched comparison was performed 100,000 times and $\Delta \mathrm{PAR}$ and $p$-values were calculated for each comparison.

\section{Heritability}

We used POLY software (http://people.virginia.edu/ $\sim$ WC9c/poly) to estimate the heritability of the physiological aging rate (PAR) based on the known family structure in the SardiNIA cohort.

\section{GWAS}

Genome-wide association studies (GWAS) have been a powerful tool to infer genetic associations with diseases and quantitative traits [60-62]. We treated PAR as a quantitative trait and employed GWAS to determine genetic loci significantly associated with PARs for the SardiNIA study.

\section{Trait ranking}

We used three different methods to rank the traits in SardiNIA and InCHIANTI. Each method was designed to assess the relative importance of a trait to a different measure of physiological aging. First, traits were scored for their association to the physiological aging rate (PAR) using the $p$-value from a two-tailed student's $t$ test comparing the mean trait values of individuals in the top PAR quartile and that of individuals in the bottom PAR quartile. The fold change of the trait values between the two quartiles was used to quantify the relation between significant traits and the PAR. Secondly, traits were scored for their association with the physiological age using the Pearson correlation $r$ between the trait values and the measured physiological age of individuals. Finally, traits were scored for their contribution to the predictive performance $\left(\mathrm{R}^{2}\right)$ of the model. Each trait was independently removed from the model, and the average loss in model performance over
500 training-testing splits was calculated with respect to a baseline performance of $\mathrm{R}^{2}=0.858$ (SardiNIA) and $\mathrm{R}^{2}=0.702$ (InCHIANTI). We refer to the difference in model performance after removing a given trait as the added value $\left(\mathrm{R}^{2}\right)$ of the trait.

\section{RESULTS}

\section{Intuition about physiological aging rate (PAR)}

We used machine learning to infer an individual's PAR (see Materials and Methods for details), the ratio between physiological age and chronological age, from their physiological profile (i.e., set of quantitative trait values) by comparing that profile to the trait values of other participants in the study ("training data"). For example, a 50-year-old man might have a physiological profile (values of body mass index, pulse wave velocity, NEOPIR assertiveness, etc.) that predicts a physiological age of 48 years. As a result, he would have $\mathrm{PAR}=48 / 50=0.96-$ i.e., aging slower than expected for his chronological age peers.

\section{Comparison of machine learning models}

We applied various machine learning models to predict age from the SardiNIA trait data. Each machine learning model was cross-validated for 10,000 different training-testing splits to ensure convergent values for individuals. Physiological age was then computed as the mean predicted age for an individual across all splits for which the individual was in the testing set. The physiological aging rate (PAR) was calculated as the ratio of physiological age to chronological age. The average PAR and median PAR are close to one (SardiNIA mean PAR $=1.04$, median PAR $=1.00)$. Slowed and accelerated aging correspond to PAR $<1$ and PAR > 1 respectively.

We used the coefficient of determination $\left(\mathrm{R}^{2}\right)$ between the actual and predicted ages to evaluate the performance of each model. Higher $\mathrm{R}^{2}$ values indicated better model performance, with most individuals having concordant chronological and physiological age. All machine learning models produced well-correlated physiological age estimates $\left(\mathrm{R}^{2}>0.8\right.$; see Figure $\left.1 \mathrm{~A}\right)$. The physiological age and PAR measurements were also highly correlated between any two machine learning models $\left(\mathrm{R}^{2}>0.9\right.$ for physiological ages; $\mathrm{R}^{2}>$ 0.7 for PARs; see Figure 1B). These similarities suggest the presence of a model-agnostic, intrinsic physiological aging signal. The top-performing model, the random forest classifier (RFC) $\left(\mathrm{R}^{2}=0.86\right.$ for the SardiNIA baseline study; see Figure 1C), reached performance saturation with 37 rank-ordered traits when all individuals were involved in training/testing, or with 
75 individuals when all traits were involved in training/testing (see Supplementary Figure 3).

Results were consistent between the baseline study and all follow-up waves of the SardiNIA study and were also consistent between all study visits in the InCHIANTI study (see Supplementary Figure 4 and Supplementary Figure 5 respectively). In the baseline study, the standard deviation in predicted age was 2.6 years for SardiNIA and 3.3 years for InCHIANTI, and standard deviation in predicted age was uncorrelated

A

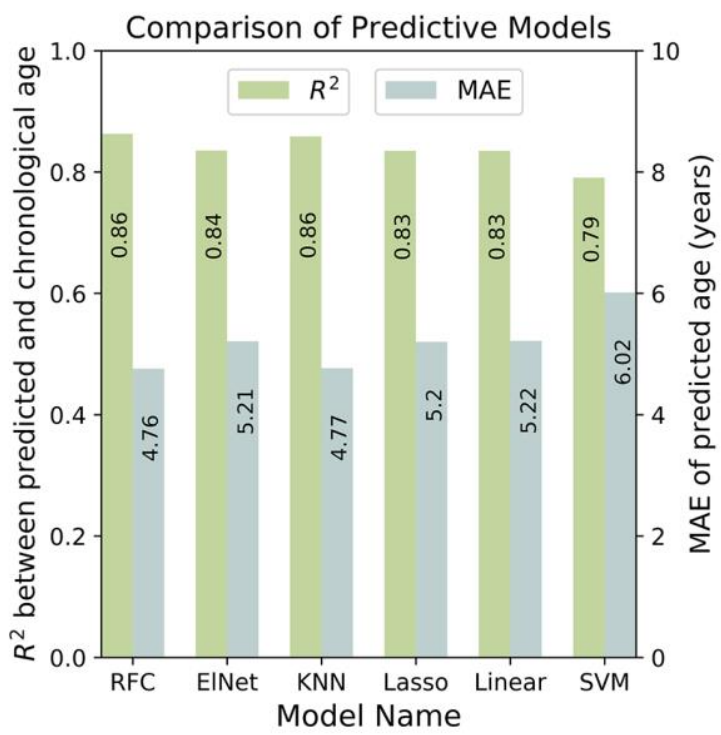

C

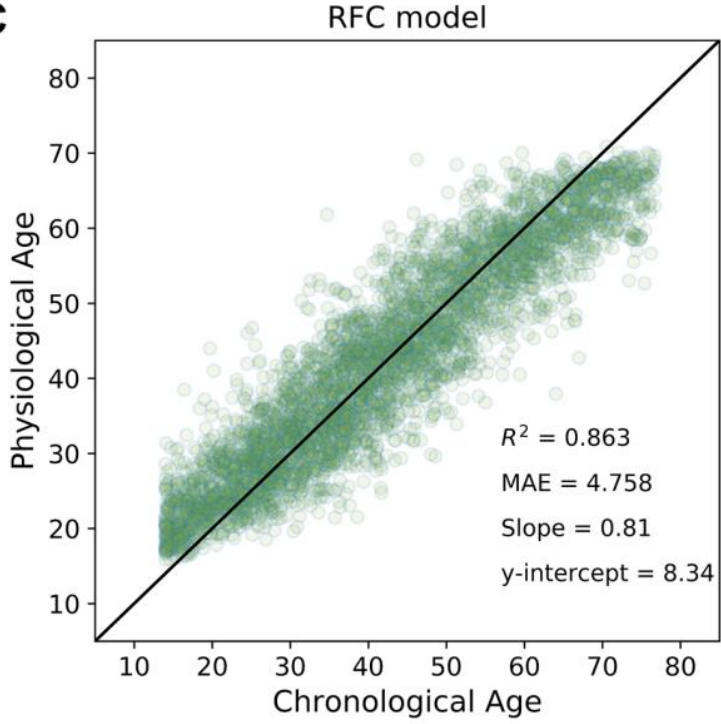

with subject age for both studies. In SardiNIA, there was no significant difference between the PAR measures of male and female study participants (see Supplementary Figure 6). The distribution of PARs obtained from the RFC model was also generally independent of age (Figure 1D) and highly similar between the SardiNIA and InCHIANTI studies despite notable differences in the chronological age range of the two cohorts (see Supplementary Materials, Supplementary Figure 7). However, the very youngest individuals were distinctly more likely to have PAR $>1$,

\section{B}
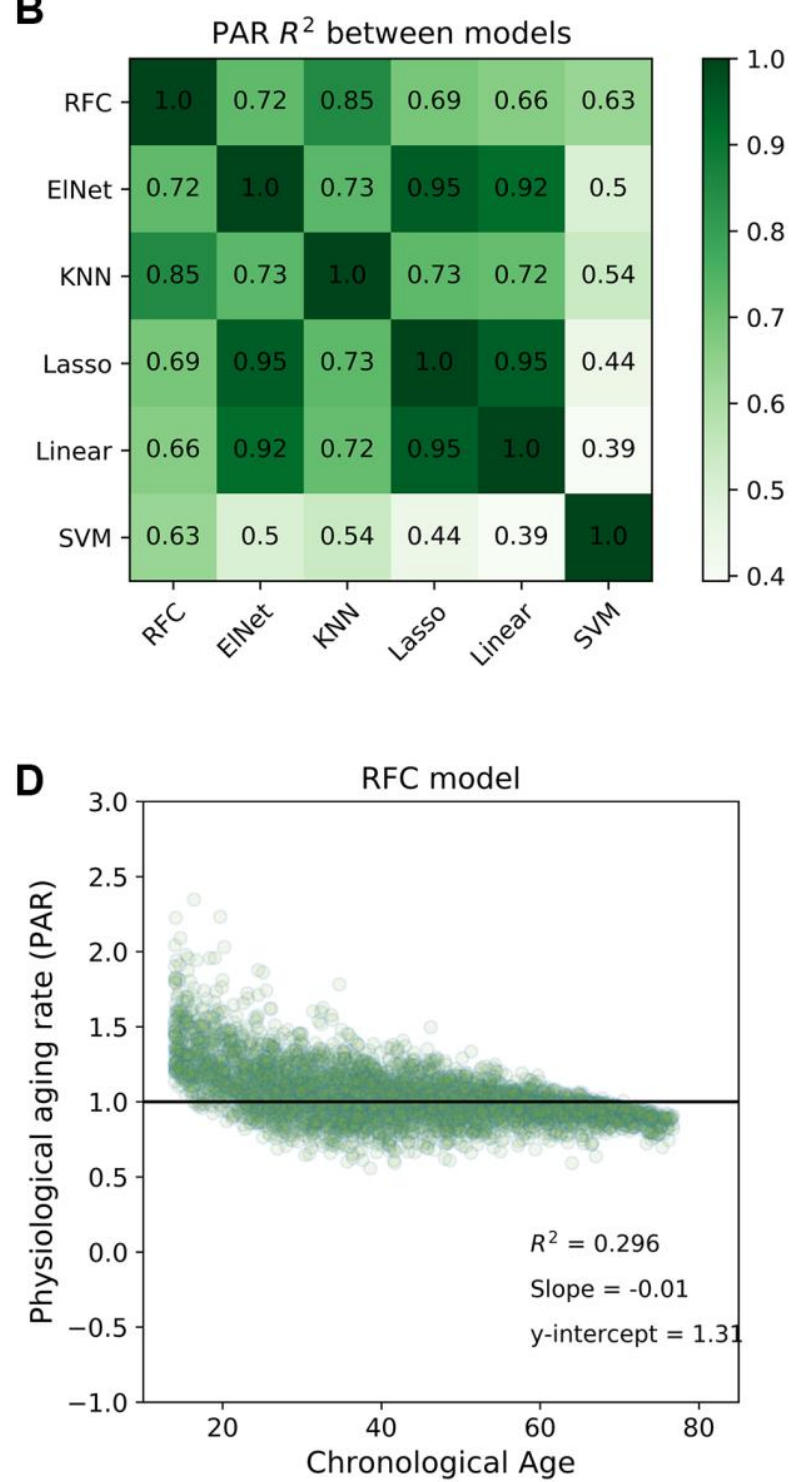

Figure 1. Predictive performance of machine learning models. (A) Comparison of the predictive performance measured by the coefficient of determination $\left(R^{2}\right)$ and mean absolute error (MAE) for all machine learning models investigated in the study. The random forest classifier (RFC) model was among the top-performing models. (B) Physiological aging rates were highly correlated between different models. Shown is a tileplot of $\mathrm{R}^{2}$ between PARs obtained from different models where darker green corresponds to higher values. (C) Physiological ages predicted by the RFC model were well-correlated with chronological ages of individuals in the SardiNIA study. (D) Physiological aging rate (PAR) of individuals obtained from the RFC model was weakly correlated with chronological age. All figures shown are for the baseline (W1) SardiNIA study. Similar results as in (C) and (D) were observed in follow-up waves of the SardiNIA study, for the elastic net regression model, and in the InCHIANTI study (see Supplementary Materials). 
and the very oldest individuals were more likely to have PAR $<1$. This trend may be due to several factors: selection bias, with individuals who survived to advanced age being more likely to have a slower intrinsic aging rate; different underlying biology for the youngest individuals who are undergoing development; or artefacts in the classification procedure caused by the absence of data for individuals younger than the youngest or older than the oldest in the cohort (see "Linear rescaling and trimming to correct for the imbalanced distribution of PARs across age" in Supplementary Methods for details on linear rescaling and/or trimming of predicted ages to reduce the PAR imbalance). Removing the youngest and oldest age bins resulted in a more balanced distribution of PARs across chronological age but did not significantly change the correlation of physiological age with chronological age in either SardiNIA or InCHIANTI (see Supplementary Figure 8).

\section{Using common traits}

Models for estimating physiological age are especially useful if they can work with commonly measured traits. Therefore, we trained the RFC model on a reduced set of SardiNIA traits that are part of routine clinical screenings. The physiological age obtained from this model was again strongly correlated with chronological age $\left(\mathrm{R}^{2}=0.78\right.$ for RFC). The addition of ten cardiovascular biomarkers that are commonly measured in cardiology specialty clinics resulted in a total of 65 traits (52 after Fisher trait selection, see Methods; full list in Supplementary Materials) and further increased model performance to a level comparable to the fulltrait model $\left(\mathrm{R}^{2}=0.85\right.$ for random forest on the baseline SardiNIA data, see Supplementary Materials). The physiological age and PAR measurements obtained from this "common-trait model" correlated well with their counterparts from the full-trait model $\left(\mathrm{R}^{2}=0.95\right.$ for physiological age; $\mathrm{R}^{2}=0.8$ for PAR, see Supplementary Figure 9). These results are consistent with a clinically relevant intrinsic process of aging (see Discussion). Results for the common-trait model were also consistent for follow-up waves of SardiNIA (see Supplementary Figure 10).

\section{Physiological aging rate is associated with mortality}

Next, we assessed the clinical relevance of the new aging rate measurement. Specifically, we tested whether PAR is associated with mortality using two methods. First, a Cox proportional hazards model showed that independent of chronological age and sex, PAR was a significant predictor for survival (263 deaths, $\sim 51,000$ person-years). As shown in Table 1, a two-fold higher aging rate was significantly associated with a 3.59 fold increase of the death hazard ( $p$-value $=0.010)$. As age and sex were included as covariates in the model, we also observed that independent of PAR, a one-year age increase would increase the hazard by 1.11 fold $\left(p\right.$-value $\left.<2 \times 10^{-16}\right)$ and that males have a hazard 1.67 times higher than females $\left(p\right.$-value $\left.=6 \times 10^{-5}\right)$. As a point of comparison, we used the well-known KlemeraDoubal method (KDM) to compute biological age using six traits: C-reactive protein, creatinine, glycated hemoglobin, total cholesterol, urea nitrogen, and systolic blood pressure [37]. Unlike PAR, the KDM biological aging rate was not significantly associated with mortality ( $p=0.53$, Cox regression with age, sex, $\log _{2}$ (KDM aging rate); 275 deaths, 26,800 personyears).

To provide more evidence for the association between PAR and mortality, we then randomly age-matched deceased individuals with living participants and compared their aging rates. We performed 100,000 randomizations and found that $77.4 \%$ of the agematched comparisons had significant differences $(p<$ $0.05)$ in the mean PAR values of the deceased and living subjects with a mean $\triangle \mathrm{PAR}$ of 0.016 (Figure 2A). Nearly all random age-matched comparisons reported $\triangle \mathrm{PAR}>0$ (Figure 2A). In contrast, the randomized control showed, as expected under null, few (5.2\%) $p$ values $<0.05$ and a mean $\triangle \mathrm{PAR}=0.00$ (Figure $2 \mathrm{~B}$ ). Furthermore, lifespans of these individuals were negatively correlated with the corresponding PARs in both the full-trait model $(r=-0.491$, Figure $2 \mathrm{C})$ and common-trait model $(\mathrm{r}=-0.469$, see Supplementary Figure 11). These results all suggest that individuals with lower PARs outlived their counterparts with higher PARs.

\section{Correlation of physiological aging rate (PAR) with epigenetic aging rate (EAR)}

DNA methylation (DNAm) ages were measured for individuals in the baseline study and the most recent follow-up study of InCHIANTI with the Horvath model [20]. Using the same method for calculating PAR, we calculated a DNA methylation-based epigenetic aging rate (EAR) as the ratio between the DNAm age and the chronological age for an individual. The mean EAR was correlated with the mean PAR $\left(\mathrm{R}^{2}=0.36, \mathrm{r}=0.6\right.$, Figure $3 \mathrm{~A}$ ) despite the two modalities representing measures of aging from separate biological levels (i.e., methylome for EAR; proteome, metabolome, and phenome for PAR). The positive correlation between EAR and PAR persisted after eliminating the effect of chronological age and sex as confounding variables (see Supplementary Figure 12). Both PAR and EAR measurements were largely uncorrelated with chronological age. In addition to DNA methylation- 
Table 1. The effect of physiological aging rate (and age and sex) on survival from a cox proportional hazards model.

\begin{tabular}{lcccc}
\hline & Coefficient & $\begin{array}{c}\text { Exp (Coefficient) } \\
\text { hazard ratio }\end{array}$ & z-statistic & $\boldsymbol{p}$-value \\
\hline Age & 0.11 & 1.11 & 15.69 & $<2 \mathrm{e}-16$ \\
Sex & 0.51 & 1.67 & 4.02 & $5.9 \mathrm{e}-5$ \\
$\log _{2}(\mathrm{PAR})$ & 1.28 & 3.59 & 2.57 & 0.010 \\
\hline
\end{tabular}

based aging rates from [20], the PAR was also positively correlated with DNA methylation-based aging rates [19], GrimAge aging rates [24], and PhenoAge aging rates [23] (see Figure 3B). We also computed the KDM biological age [37] using eight markers from InCHIANTI, which were C-reactive protein, creatinine, glycated hemoglobin, albumin, total cholesterol, alkaline phosphatase, urea nitrogen, and systolic blood pressure. The PAR was better correlated than KDM biological aging rate with all DNA methylation-based aging rates except PhenoAge, which
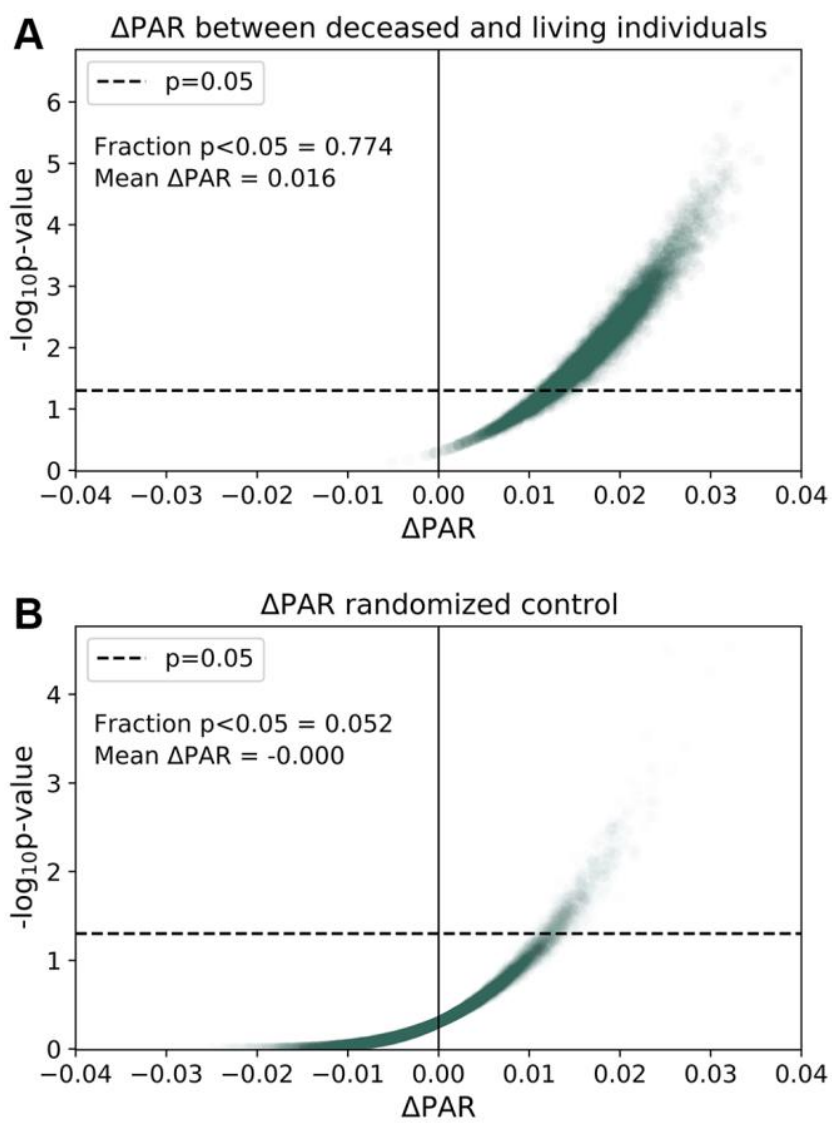

suggests that the PAR is a relatively good proxy for DNA methylation-based aging measures.

\section{Heritability and GWAS of physiological aging rate}

We estimated the extent to which PAR is heritable using pedigrees. PARs from the full-trait RFC model observed moderate heritability $\left(\mathrm{h}^{2}=0.33\right)$, and PARs obtained using the common-trait model were similarly heritable $\left(h^{2}=0.30\right)$. These values were comparable to previous estimates for the heritability of aging and

\section{C}

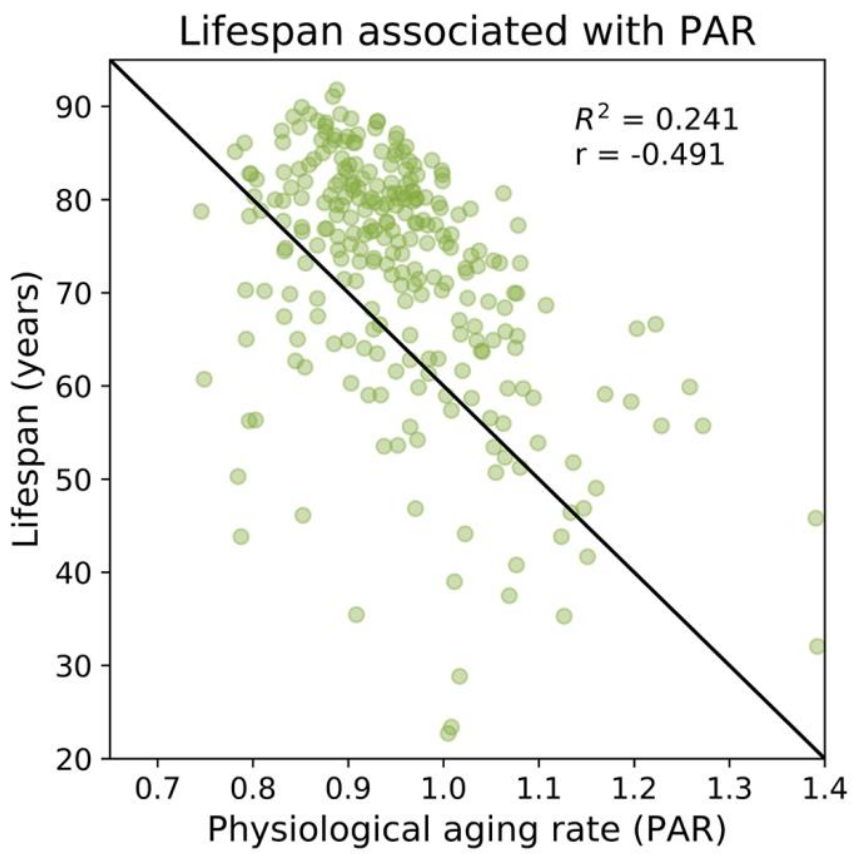

Figure 2. Physiological aging rates are associated with mortality. (A) Age-matched mortality analysis: 265 deceased participants were randomly paired with age- Matched \pm 0.5 years) living participants in the baseline SardiNIA study. We calculated the difference in the mean PAR measurements of the two groups, $\triangle P A R=P A R_{\text {deceased }}-P \mathrm{PR}_{\text {living }}$ and the corresponding $p$-value from a one-sided, one-sample $t$ test for $\triangle P A R>0$.The age-matched grouping was performed 10000 times and $\triangle P A R$ and $p$-values were calculated for each of the 10000 comparisons. $77.4 \%$ of the age-matched comparisons produced significantly greater than zero $\triangle P A R$ values $(p<0.05)$ and the mean $\triangle P A R$ across all comparisons was 0.016 . Nearly all comparisons ( $>99 \%)$ had $\triangle P A R>0$, which indicated that PAR deceased $>P A_{\text {living }}$ on average. (B) Randomized, age-matched control comparisons produced a $5.2 \%$ frequency of significantly greater than zero $(p<0.05) \triangle P A R$ values and the mean $\triangle P A R$ was 0.00 . Consistent with random assignment, $50.8 \%$ of the $\triangle P A R$ values were greater than zero. (C) Lifespans for individuals were negatively correlated with PARs $(r=-0.491)$. 
longevity (Karasik et al., 2005). Furthermore, modifications of the PAR measurements such as linear rescaling of physiological ages increased heritability up to $\mathrm{h}^{2} \sim 0.45$ (see Supplementary Materials and Supplementary Figure 13; see Supplementary Figure 14 for linearly rescaled physiological age results). Like typical traits, the physiological aging rate, a proxy for the intrinsic aging process, appears to have a significant genetic component.

The heritability of PAR encouraged us to perform genome-wide association studies (GWAS) in SardiNIA using PAR as a quantitative trait. GWAS identified two genome-wide significant loci [ 16 significant $(p<5.00 \mathrm{E}-$ $8)$ single-nucleotide polymorphisms] associated with PAR. Six SNPs fell in the CFI/GARI locus located on chromosome 4 (Figure 4A, most significant CFI/GARI SNP with $p=5.55 \mathrm{E}-10$ ), which has already been linked to aging in earlier studies (see Discussion). CFI/GARI also harbored the most significant SNP associated with physiological age acceleration (PAA), another measure of age progression ( $p=4.07 \mathrm{E}-9)$ (see Supplementary Materials), and was among the top SNPs associated with the PAR from the common-trait model $(p=$ 1.731E-7) and with other machine learning models (e.g., elastic net regression model, $p=2.95 \mathrm{E}-7$, see Supplementary Materials).

Three significant SNPs and seven significant proximally located SNPs $(p<5.00 \mathrm{E}-8)$ were observed at the second locus, LINC00202, also referred to as FAM238C (Figure 4B, most significant LINC00202 SNP with $p=$ 3.94E-10). The LINC00202 locus also contained the top SNPs for PAR measurements from the common-trait model ( $p=1.100 \mathrm{E}-8)$ and from other machine learning models (e.g., elastic net regression, $p=2.153 \mathrm{E}-10$ ). LINC00202 is a long intergenic non-coding RNA with largely uncharacterized function (see Discussion). Other genes with suggestive $p$-values for possible associations with PAR $(p<5.00 \mathrm{E}-7)$ include APLF, ARHGAP15, SCD5, SOGA2, HIVEP1, ANKRD26, DYNC2LII, ZNF518B, TMEM45A, CLDN10, and CSMD1 (see Supplementary Materials for a discussion of these genes).

\section{Trait ranking and biomarkers of aging}

We ranked the traits in the SardiNIA study and separately for the InCHIANTI study using three distinct methods (see Methods). These included measuring the difference in trait values between the top and bottom PAR quartiles (Figure 5A), correlation of traits with physiological age (Figure 5B), and the additional contribution of each trait to model performance (Figure 5C), which is similar to variable importance for random forest models [63]. Although, as outlined in the Methods, the sets of traits used in SardiNIA and InCHIANTI were not identical, the top ranked traits showed general concordance in the two studies. For example, a set of cardiovascular traits were ranked as

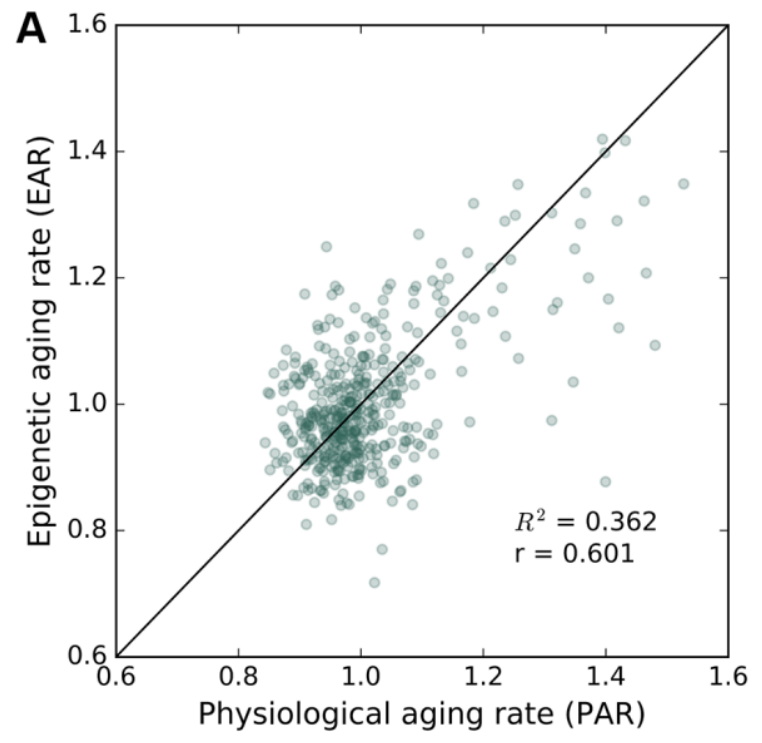

B

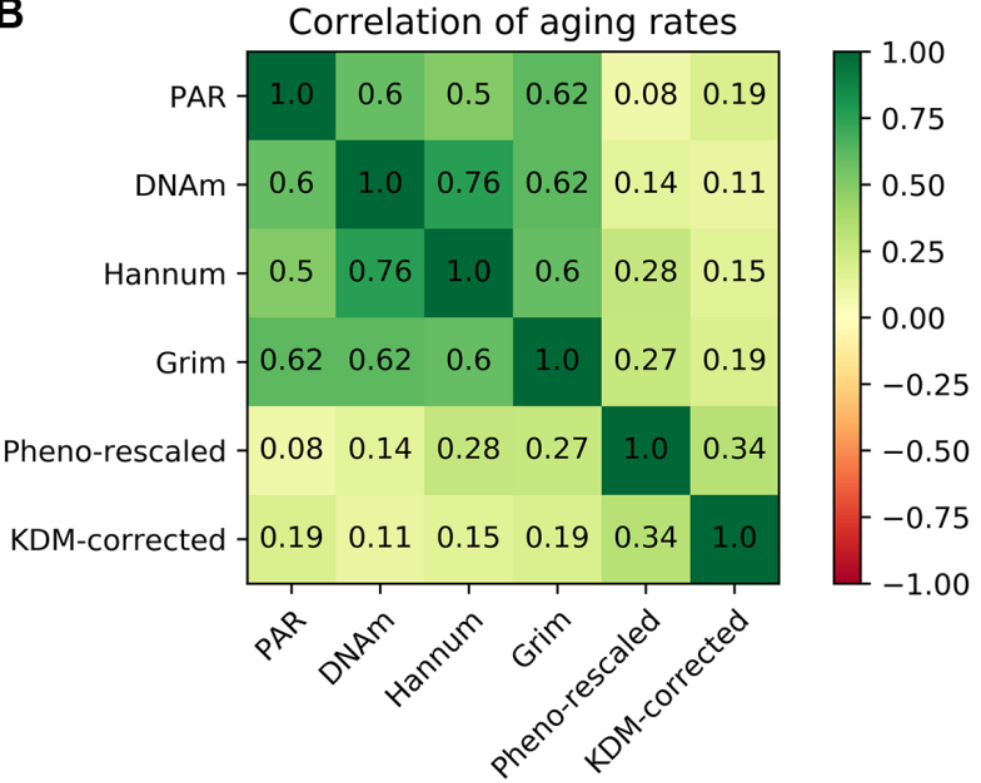

Figure 3. Correlation between epigenetic aging rate and physiological aging rate. Correlation between PAR and other aging rate measures. (A) Mean physiological aging rates (PARs) obtained from physiological age measurements were correlated $\left(R^{2}=0.362, r=0.601\right)$ with the mean epigenetic aging rates (EARs) calculated for the same individuals across the baseline and latest follow-up InCHIANTI studies using the Horvath DNAm age. (B) Pearson correlation values between different pairings of aging rate measures including PAR, Horvath DNAm age, the Hannum DNAm age, GrimAge, rescaled PhenoAge, and the corrected KDM biological age with eight covariates. 
top traits in both cohorts. In SardiNIA, CCA intima media thickness, pulse wave velocity, and diastolic CCA diameter were highly ranked across all three methods (Figure 5). Several other cardiovascular traits also received high scores, including peak systolic velocity, integral time velocity, end diastolic velocity, and mean supine systolic blood pressure. Most of these traits were also highly ranked among the set of common and cardiovascular SardiNIA traits (Spearman correlation of $\rho=0.92$, see Supplementary Figure 15), corroborating the comparable predictive performance between the common-trait and the full-trait models. The prevalence of high-scoring cardiovascular traits was likewise seen in the InCHIANTI study cohort, including
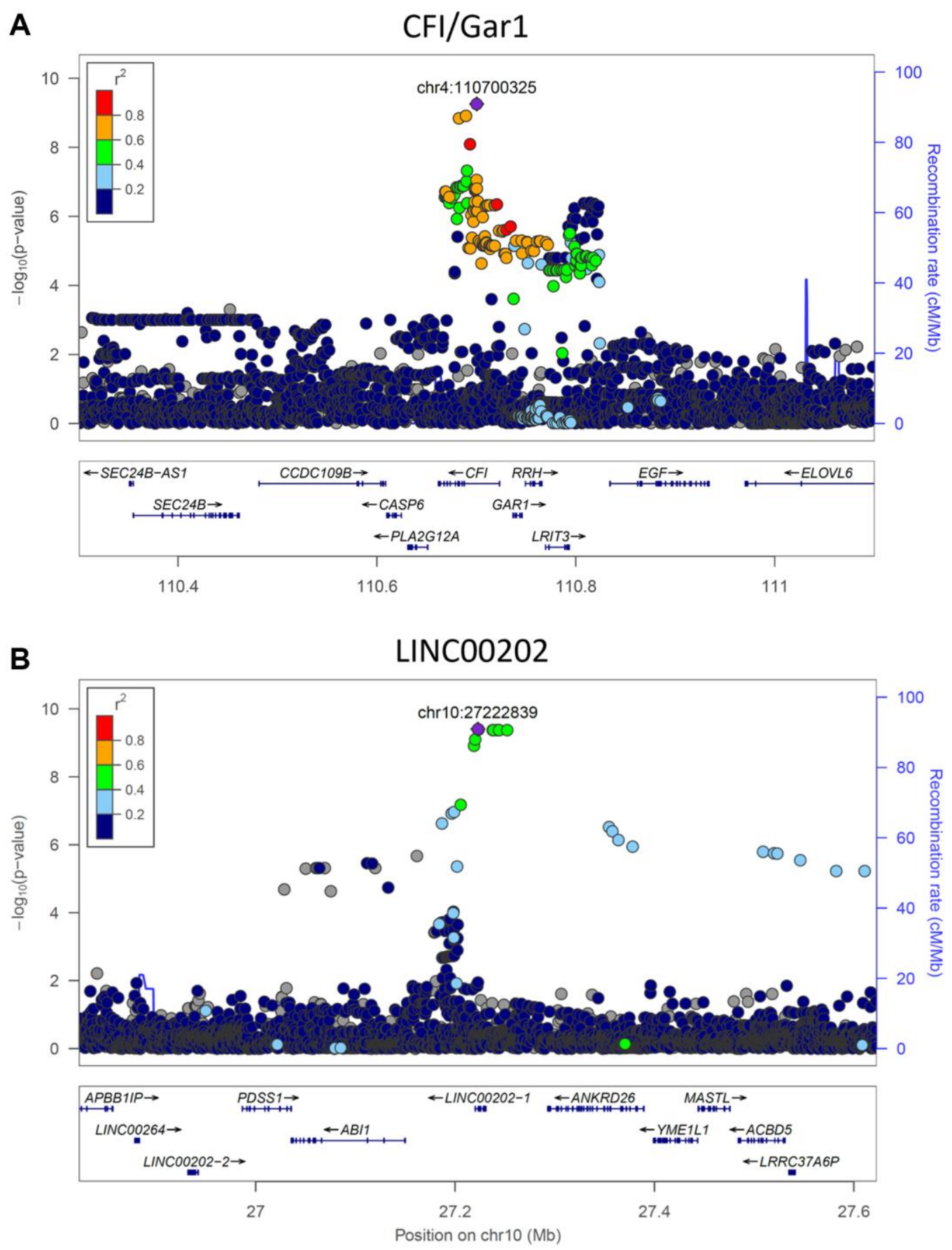

Figure 4. Significant genetic loci obtained from genome-wide association study. (A) CFI/GAR1 was significantly associated with the physiological aging rate (PAR). CFI is a complement factor and has been linked to age-related macular degeneration and other agerelated disorders. GAR1 is an accessory protein for the active telomerase complex and is an eQTL target of the top CFI/GAR1 SNPs. (B) LINC00202 was significantly associated with the PAR and corresponded to a long non-coding RNA that has been indirectly linked to agerelated disease. Plots were made using LocusZoom (Pruim et al., 2010). 
pulse wave velocity, systolic blood pressure, and repolarization phase among the top 5\% of ranked traits.

Some NEOPIR (Revised NEO Personality Inventory) personality traits [64] were highly ranked in SardiNIA, including fantasy, compliance, excitement seeking, and assistance with personality test. Other highly ranked SardiNIA traits across multiple methods included general measures of obesity such as waist circumference and body mass index, and blood molecular markers such as cholesterol, nitrogen, glucose, sodium, uric acid, and alanine aminotransferase levels. Many of these traits were also present among the highest-ranking InCHIANTI traits (see Supplementary Figure 16: in the top 5\%: blood nitrogen, creatinine, sodium intake, hypertension; in the top 20\%: cholesterol, IL-6, fibrinogen; and in the top
30\%: uric acid, transferrin, waist circumference). In particular, creatinine level was highly ranked by added value (R2) to model performance and was also the highest-ranked trait in the InCHIANTI study. InCHIANTI trait rankings (see Methods) using the epigenetic age and EAR resulted in similar sets of highranked features (see Supplementary Figure 17). Most of the top traits from each scoring method have been suggested earlier as biomarkers of aging, and others may become candidate biomarkers (see Supplementary Materials for full rankings of traits).

\section{DISCUSSION}

We present machine learning as a promising framework for measuring physiological age from broad-ranging physiological, cognitive, and molecular traits.
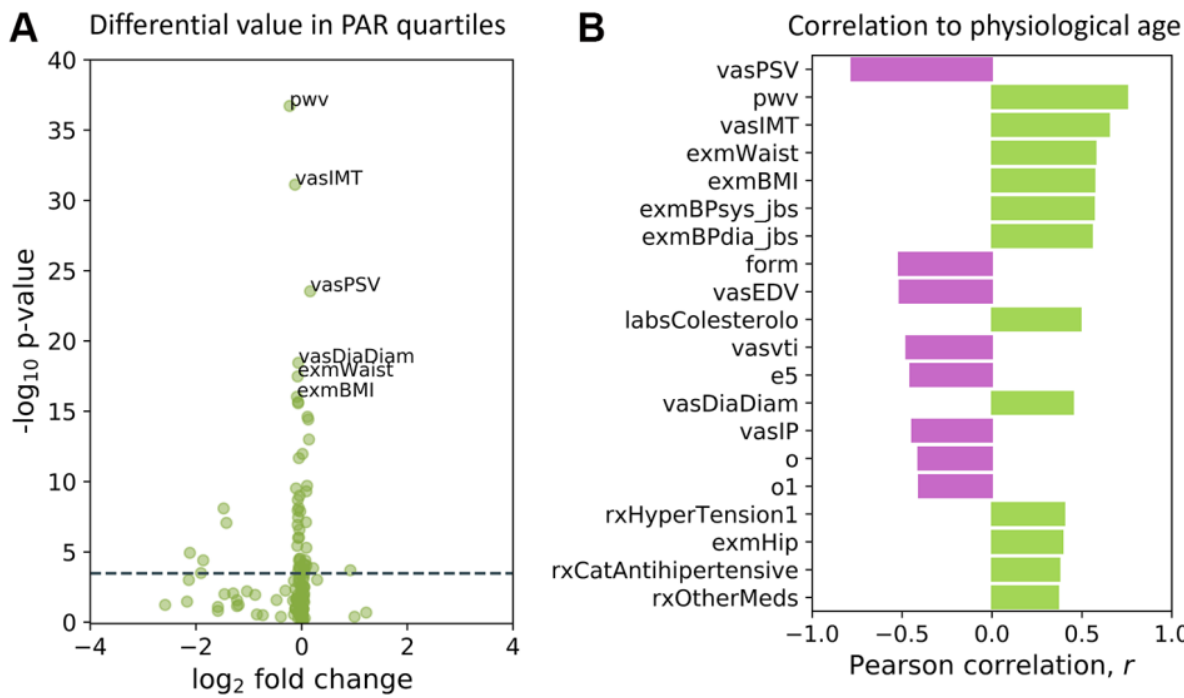

\author{
pwv - Pulse wave velocity \\ vasIMT - CCA intima media thickness \\ vasPSV - Peak systolic velocity \\ vasDiaDiam - Diastolic CCA diameter \\ vasEDV - End diastolic velocity \\ Vasvti - Integral time velocity \\ vasIP - Pulsatility index \\ vasAT - Acceleration time \\ exmWaist - Waist circumference \\ exmBMI - Body mass index \\ exmHip - Hip circumference \\ exmBPsys jbs - Supine blood \\ pressure (systolic) \\ exmBPdia_jbs - Supine blood \\ pressure (diastolic) \\ labsColesterolo - Serum cholesterol \\ level \\ labsAzotemia - BUN \\ labsGammaGT - Gamma- \\ glutamyltransferase \\ labsGlicemia - Serum glucose level \\ labsSodiedemia - Serum sodium level \\ labsInsulinemia - Serum insulin level \\ labsAST - Aspartate aminotransferase \\ labsALT - Alanine aminotransferase \\ rxHyperTension 1 - Hypertension \\ medication? \\ rxCatAntihipertensive - \\ Antihypertensive medication? \\ rxOtherMeds - Other medication? \\ o- NEOPIR openness \\ o1 - NEOPIR fantasy
}

Figure 5. Top traits determined by three independent methods. (A) Volcano plot of the top traits in the full-trait model, which included pulse wave velocity (pwv), CCA intima media thickness (vasIMT), peak systolic velocity (vasPSV), diastolic CCA diameter (vasDiaDiam), waist circumference (exmWaist), and body mass index (exmBMI). Significant differences between the mean trait values of subjects in the top and bottom PAR quartiles were determined using a two-tailed students $t$-test on each trait. The dotted line corresponds to a Bonferroni-corrected threshold of $p=3.33 \times 10^{-4}$ calculated from single-test threshold of $p=5.00 \times 10^{-2}$. Many top traits were also highly ranked in the common-trait model. (B) Traits rank-ordered by Pearson correlation ( $r$ ) with physiological age measured using the fulltrait RFC model. (C) Traits rank-ordered by approximate added value $\left(R^{2}\right)$ for the full-trait RFC model in SardiNIA $\left(R^{2}=0.858\right.$ with all traits used in model). Added value $\left(R^{2}\right)$ was averaged over 500 training-testing iterates for each trait. There was significant overlap in the highest ranked traits across all three scoring methods and between the common-trait and full-trait models (see Supplementary Materials). Trait names are as they appear in the SardiNIA study; descriptions of all traits are available in the Supplementary Materials. 


\section{Physiological aging rate model}

The measured physiological age is well-correlated with chronological age $\left(\mathrm{R}^{2}>0.8\right)$, and different sets of traits give very comparable estimates of physiological aging rate (PAR). Furthermore, PAR calculated from the traits is correlated with the epigenetic aging rate (EAR) $\left(\mathrm{R}^{2}=0.36, \mathrm{r}=0.6\right)$, even though the two aging rates are based on separate biological frameworks. Comparisons with aging rates derived from newer epigenetic age models, such as GrimAge [24] and phenotypic epigenetic age [22], could provide further insight into this relationship.

The physiological age estimates obtained from the model are well-correlated with chronological age and comparable to epigenetic age measures. The approach described here joins other recent attempts to quantify "aging" from functional parameters. It is encouraging that our results correlate with other approaches that are also predictive of risk of mortality. In particular, the $\mathrm{PAR}$, as compared to the KDM biological aging rate, is better correlated with GrimAge, the epigenetic age score with the strongest mortality association [65]. Unlike many earlier studies on aging metrics, we show that PAR is longitudinally stable for an individual (see Supplementary Figure 18), with temporal correlation comparable to that of epigenetic age acceleration indices [66] and higher for shorter time differences, which is a necessary and desirable property for biological age measurements $[3,5,6]$. Also, because PAR predictions are derived from multiple physiological, cognitive, and molecular inputs that span the proteome, metabolome, and phenome, they potentially represent a larger fraction of the manifestations of aging than earlier models. The model maintains high predictive performance even after reducing the trait set to 65 commonly accessible markers. These traits can be efficiently and inexpensively measured in many clinics already equipped to collect them-making the model framework, like some other current models [37], easily adaptable for clinical studies and workflows.

We focused primarily on the random forest classifier model in this investigation, but all machine learning models detected a strong physiological aging signal $\left(\mathrm{R}^{2}\right.$ $>0.80$ ). The strength of the signal corroborates the presence of a central aging effect that exists independently of model type. This is an idea that has been previously explored in theoretical models $[67,68]$ and studies where temporal scaling of a central aging rate was observed [69]. The top traits we found in both SardiNIA and InCHIANTI studies are manifest in many tissues and organs. The findings are thus consistent with the likelihood that PAR represents the rate of a pathology-independent aging process - an answer to Medawar's famous question [1].

Top traits identified through various methods recapitulate previously identified associations between physiological aging and/or age-related disease and cardiovascular function [70-72], molecular markers, physiological measurements, and personality features. A few novel exceptions are promising candidates for new biomarkers of aging (e.g., NEOPIR compliance and agreeableness, step-climbing endurance, and hand tapping coordination).

\section{PAR as a predictor of survival}

Improved prediction of chronological age is not necessarily associated with increased prediction of mortality [73]. We used two methods to test directly for association between PAR and mortality/survival. As shown by a Cox proportional hazards model, a two-fold increase of the aging rate would increase the risk of dying by 3.59 fold, or equivalently, a $10 \%$ increase of aging rate would increase the risk by 1.19 fold. Our second method showed that in 100,000 randomizations, deceased individuals had significantly higher PARs $(p<0.05)$ than agematched living subjects $77.4 \%$ of the time. These results clearly show the effect of aging rate on survival and thus a prospective benefit of slowing aging rates. In the same Cox survival model, we also observed the effects of age and sex on survival (e.g., a one-year age increase would increase the risk of dying by 1.11 fold on average, and males have a risk 1.67 times higher than females of the same-age). These results are consistent with numbers from other resources. For example, based on the 2017 Actuarial Life Table published by US Social Security Administration, a 50-year-old man has a death probability 1.09 times higher than a 49 -year-old man, and a 50-year-old man has a death probability 1.60 times higher than a 50-year-old woman. These consistent results support the validity of the apparent effect of PAR on survival. In comparison, aging rates computed using the Klemera-Doubal method, another non-epigenetic biological age model, were not significantly associated with mortality.

\section{Heritability and GWAS signals for PAR}

The heritability of PAR ( $30 \%)$ implies a significant genetic contribution to an individual's aging rate. Genome-wide association studies identified the CFI/GARI and LINC002O2 loci to be significantly associated with the physiological aging rate. Both loci have also been previously associated with aging and age-related disease. 
Complement factor I (CFI) is involved in eliminating pathogens, triggering inflammation, and removing debris from cells and tissues [74-76]. Variants in the CFI gene have been linked to age-related macular degeneration (AMD) [75, 77-80], chronic infection [81], and broader aging processes [77, 82].

But intriguingly, GAR1, encoded by the other candidate gene at the locus, is one of four accessory proteins associated with the telomerase ribonucleoprotein (RNP) complex [83]. GARl polymorphisms have been associated with differences in acute heart rate response to exercise, which is a predictor of all-cause mortality and cardiovascular mortality [84]. Other components and effectors of telomerase have also been linked to aging $[60,62,85,86]$.

We retrieved information on significant single-tissue expression quantitative trait loci (eQTLs) for $C F I$ and GAR1 mRNAs from the GTEx database [87]. All significant CFI/GARl SNPs corresponding to significant eQTLs were associated with the expression level of GARI mRNA, but not with that of CFI. Further, GARI mRNA was the most significantly associated $(p=6.5 \mathrm{E}-$ 13) for the eQTL corresponding to the top CFI/GARI SNP (rs11940869) across all tissues. Significant eQTLs corresponding to rs11940869 were found in several tissue types, including brain (frontal cortex, caudate, putamen) and esophagus mucosa (see Supplementary Materials). Furthermore, we analyzed RNA-seq gene expression profiles of tissues from six diverse GTEx tissues (heart, lung, liver, thyroid, colon, cerebellum). GARI mRNA levels were reduced with age in heart $(\mathrm{r}=$ $-0.135)$, lung $(\mathrm{r}=-0.313)$, and colon $(\mathrm{r}=-0.231)$ tissues, whereas CFI mRNA expression levels did not correlate substantially with age in most human organs (see Supplementary Figure 19). These results suggest that it may not be coincidental that telomerase, which is associated with GAR1, was determined to be the critical factor that prevents replicative senescence in the fibroblast model for aging: the supply of active telomerase to aging cells promotes their indefinite growth and division, without activation of aging-related SASP (senescence-associated secretory phenotype) proteins. Our data suggest that effects on telomerase activity and possibly related effects on chromatin are not only markers for aging but may be part of the intrinsic mechanism, implicating cell senescence.

As for the other genome-wide significant signal in LINC00202, expression quantitative trait loci affecting LINC00202 expression have been significantly associated with age-related endophenotypes in earlier genome-wide association studies [88]. Although the role of most lncRNAs remains unknown, a number of IncRNAs have been shown to influence molecular processes that contribute to age-related phenotypes [89], and are known to influence age-related cardiovascular disease [90], oxidative stress responses and cellular senescence [91], and many age-related debilitations [92]. However, an exact role of LINC00202 in the aging process remains to be determined.

\section{Possible next steps}

We used data from two independent longitudinal studies (SardiNIA and InCHIANTI) with relatively homogenous populations. Whether the significantly PAR-associated loci and highest-scoring traits reported in this investigation are reproducible in more heterogenous populations must be investigated. Further hyperparameter tuning and recent developments in machine learning for handling sparse data [93] may also be of value in maximizing the utility of trait data for age prediction. Although PAR is measured from molecular, physiological, and cognitive traits, inclusion of traits from other data modalities may improve the metric. Several modalities that are aging-associated and potentially useful for PAR measurement include human transcriptome profiles [45, 94, 95], proteome profiles [46, 47], and image data [42, 43]. One natural extension of our method, which is based on cross-sectional trait data, is to use longitudinal data to predict a physiological age at a subsequent visit (e.g., to predict the aging rate at a subsequent visit based on the change of traits over time or based directly on the aging rates predicted at previous visits). This involves new method development and deserves efforts from a separate study.

Although we have emphasized the genetic contribution to aging rates, the majority of PAR is still attributable to environmental factors, including the effect of pathology. Further investigation of correlations between PAR and smoking or age-related diseases such as diabetes, neurodegenerative disease, cancer, and cardiovascular disease could further aid in characterizing the highestscoring traits and delineating potential aging-related pathways. In turn, these analyses may offer additional clinical utility for PAR in assessing the personalized risk and natural history of patients for each disease.

The efficacy of treatments aimed at slowing the aging process has traditionally been evaluated using individual biomarkers or limited collections of related biomarkers. Our current study has shown that PAR is a significant predictor for survival and correlated with epigenetic aging rate, providing evidence for a good measurement of "aging". Therefore, we see two potential clinical applications of this intuitive metricthe physiological aging rate: 1) physicians might evaluate the efficacy of treatments and interventions on the aging process by the change of PAR before and after 
the treatments; 2) based on the values of traits that contribute to the aging rate, physicians can make recommendations to individuals aimed at slowing the aging process (e.g., making specific diet changes, doing specific exercises). Notably, the recommendations would be much the same that are currently recommended by physicians, but the additional link of PAR to risk of mortality might motivate patients to overcome the typical reluctance to modify their regimen.

\section{AUTHOR CONTRIBUTIONS}

Conceived and designed the study: EDS, LF, DS, IG, JD. Developed the method and analyzed the data: EDS, YQ, RO, TJB, JZ, LF, DS, IG, JD. Presided the cohort studies and provided cohort data: CS, FC, SB, GRA, MG, LF, DS. Contributed materials/analysis tools: BHC, TT, JK. Wrote the paper: EDS, LF, DS, IG, JD.

\section{ACKNOWLEDGMENTS}

We thank all the volunteers of the SardiNIA and InCHIANTI projects who generously participated in the study and made this research possible.

\section{CONFLICTS OF INTEREST}

The authors have declared that no conflicts of interest exist that are related to the subject matter of the manuscript.

\section{FUNDING}

This research was supported by the Intramural Research Program of the National Institute on Aging, NIH. The InCHIANTI study was funded by the U.S. National Institute on Aging (Contract: N01-AG-50002). The SardiNIA study was funded by the National Institute on Aging (Contract: N01-AG-1-2109) and by Sardinian Autonomous Region (L.R. no. 7/2009) grant cRP3-154.

\section{REFERENCES}

1. Medawar PB. An Unsolved Problem in Biology. HK Lewis Co. 1952.

2. Baker GT 3rd, Sprott RL. Biomarkers of aging. Exp Gerontol. 1988; 23:223-39.

https://doi.org/10.1016/0531-5565(88)90025-3 PMID: $\underline{3058488}$

3. Ferrucci L, Gonzalez-Freire M, Fabbri E, Simonsick E, Tanaka T, Moore Z, Salimi S, Sierra F, de Cabo R. Measuring biological aging in humans: A quest. Aging Cell. 2020; 19:e13080. https://doi.org/10.1111/acel.13080

PMID:31833194

4. Sprott RL. Biomarkers of aging and disease: introduction and definitions. Exp Gerontol. 2010; 45:2-4. https://doi.org/10.1016/i.exger.2009.07.008 PMID:19651201

5. Khan SS, Singer BD, Vaughan DE. Molecular and physiological manifestations and measurement of aging in humans. Aging Cell. 2017; 16:624-33. https://doi.org/10.1111/acel.12601 PMID:28544158

6. Kuo PL, Schrack JA, Shardell MD, Levine M, Moore AZ, An Y, Elango P, Karikkineth A, Tanaka T, de Cabo R, Zukley $\mathrm{LM}$, AlGhatrif $\mathrm{M}$, Chia $\mathrm{CW}$, et al. A roadmap to build a phenotypic metric of ageing: insights from the Baltimore Longitudinal Study of Aging. J Intern Med. 2020; 287:373-94. https://doi.org/10.1111/joim.13024 PMID: $\underline{32107805}$

7. Ferrucci L, Levine ME, Kuo PL, Simonsick EM. Time and the Metrics of Aging. Circ Res. 2018; 123:740-44. https://doi.org/10.1161/CIRCRESAHA.118.312816 PMID:30355074

8. Jylhävä J, Pedersen NL, Hägg S. Biological Age Predictors. EBioMedicine. 2017; 21:29-36. https://doi.org/10.1016/i.ebiom.2017.03.046 PMID:28396265

9. Shamir L. Composite Aging Markers Can Be Used for Quantitative Profiling of Aging. Gerontology. 2015; 62:66-68.

https://doi.org/10.1159/000433466 PMID:26088420

10. Behrens $Y L$, Thomay $K$, Hagedorn $M$, Ebersold J, Henrich L, Nustede R, Schlegelberger B, Göhring G. Comparison of different methods for telomere length measurement in whole blood and blood cell subsets: Recommendations for telomere length measurement in hematological diseases. Genes Chromosomes Cancer. 2017; 56:700-08.

https://doi.org/10.1002/gcc.22475

PMID:28593741

11. Benetos A, Okuda K, Lajemi M, Kimura M, Thomas F, Skurnick J, Labat C, Bean K, Aviv A. Telomere length as an indicator of biological aging: the gender effect and relation with pulse pressure and pulse wave velocity. Hypertension. 2001; 37:381-85.

https://doi.org/10.1161/01.hyp.37.2.381 PMID:11230304

12. Bramwell JC, Hill AV. The velocity of pulse wave in man. Proc R Soc Lond B. 1922; 93:298-306. https://doi.org/10.1098/rspb.1922.0022

13. Hochstrasser T, Marksteiner J, Humpel C. Telomere 
length is age-dependent and reduced in monocytes of Alzheimer patients. Exp Gerontol. 2012; 47:160-63.

https://doi.org/10.1016/i.exger.2011.11.012 PMID:22178633

14. Jiang $H$, Schiffer $E$, Song $Z$, Wang J, Zürbig $P$, Thedieck K, Moes S, Bantel H, Saal N, Jantos J, Brecht M, Jenö $P$, Hall $M N$, et al. Proteins induced by telomere dysfunction and DNA damage represent biomarkers of human aging and disease. Proc Natl Acad Sci U S A. 2008; 105:11299-304.

https://doi.org/10.1073/pnas.0801457105 PMID:18695223

15. Rantanen $T$, Masaki K, Foley D, Izmirlian G, White L, Guralnik JM. Grip strength changes over $27 \mathrm{yr}$ in Japanese-American men. J Appl Physiol (1985). 1998; 85:2047-53.

https://doi.org/10.1152/jappl.1998.85.6.2047 PMID: $\underline{9843525}$

16. Studenski S, Perera S, Patel K, Rosano C, Faulkner K, Inzitari M, Brach J, Chandler J, Cawthon P, Connor EB, Nevitt M, Visser M, Kritchevsky S, et al. Gait speed and survival in older adults. JAMA. 2011; 305:50-58.

https://doi.org/10.1001/jama.2010.1923 PMID:21205966

17. Wilkinson IB, Cockcroft JR, Webb DJ. Pulse wave analysis and arterial stiffness. J Cardiovasc Pharmacol. 1998 (Suppl 3); 32:S33-37.

PMID: $\underline{9883745}$

18. Fries JF. Aging, natural death, and the compression of morbidity. 1980. Bull World Health Organ. 2002; 80:245-50.

PMID:11984612

19. Hannum G, Guinney J, Zhao L, Zhang L, Hughes G, Sadda S, Klotzle B, Bibikova M, Fan JB, Gao Y, Deconde R, Chen M, Rajapakse I, et al. Genome-wide methylation profiles reveal quantitative views of human aging rates. Mol Cell. 2013; 49:359-67.

https://doi.org/10.1016/i.molcel.2012.10.016 PMID:23177740

20. Horvath S. DNA methylation age of human tissues and cell types. Genome Biol. 2013; 14:R115.

https://doi.org/10.1186/gb-2013-14-10-r115 PMID:24138928

21. Chen BH, Marioni RE, Colicino E, Peters MJ, WardCaviness CK, Tsai PC, Roetker NS, Just AC, Demerath EW, Guan W, Bressler J, Fornage M, Studenski S, et al. DNA methylation-based measures of biological age: meta-analysis predicting time to death. Aging (Albany NY). 2016; 8:1844-65.

https://doi.org/10.18632/aging.101020

PMID:27690265
22. Levine ME, Lu AT, Quach A, Chen BH, Assimes TL, Bandinelli S, Hou L, Baccarelli AA, Stewart JD, Li Y, Whitsel EA, Wilson JG, Reiner AP, et al. An epigenetic biomarker of aging for lifespan and healthspan. Aging (Albany NY). 2018; 10:573-91.

https://doi.org/10.18632/aging.101414 PMID:29676998

23. Liu Z, Kuo PL, Horvath $S$, Crimmins E, Ferrucci L, Levine $M$. A new aging measure captures morbidity and mortality risk across diverse subpopulations from NHANES IV: A cohort study. PLoS Med. 2018; 15:e1002718.

https://doi.org/10.1371/journal.pmed.1002718 PMID:30596641

24. Lu AT, Quach A, Wilson JG, Reiner AP, Aviv A, Raj K, Hou L, Baccarelli AA, Li Y, Stewart JD, Whitsel EA, Assimes TL, Ferrucci L, Horvath S. DNA methylation GrimAge strongly predicts lifespan and healthspan. Aging (Albany NY). 2019; 11:303-27.

https://doi.org/10.18632/aging.101684 PMID:30669119

25. Marioni RE, Shah S, McRae AF, Chen BH, Colicino E, Harris SE, Gibson J, Henders AK, Redmond P, Cox SR, Pattie A, Corley J, Murphy L, et al. DNA methylation age of blood predicts all-cause mortality in later life. Genome Biol. 2015; 16:25. https://doi.org/10.1186/s13059-015-0584-6 PMID:25633388

26. Petkovich DA, Podolskiy DI, Lobanov AV, Lee SG, Miller RA, Gladyshev VN. Using DNA Methylation Profiling to Evaluate Biological Age and Longevity Interventions. Cell Metab. 2017; 25:954-60.e6. https://doi.org/10.1016/i.cmet.2017.03.016 PMID:28380383

27. Zheng $Y$, Joyce $B T$, Colicino $E$, Liu L, Zhang $W$, Dai $Q$, Shrubsole MJ, Kibbe WA, Gao T, Zhang Z, Jafari N, Vokonas P, Schwartz J, et al. Blood Epigenetic Age may Predict Cancer Incidence and Mortality. EBioMedicine. 2016; 5:68-73.

https://doi.org/10.1016/j.ebiom.2016.02.008 PMID:27077113

28. Declerck K, Vanden Berghe W. Back to the future: Epigenetic clock plasticity towards healthy aging. Mech Ageing Dev. 2018; 174:18-29.

https://doi.org/10.1016/i.mad.2018.01.002 PMID:29337038

29. Chen HH. Impact of Natural Sequence Variation on Aging in the Recombinant Inbred Lines of Caenorhabditis Elegans. ProQuest. 2008.

30. López-Otín C, Blasco MA, Partridge L, Serrano M, Kroemer G. The hallmarks of aging. Cell. 2013; 153:1194-217. 
https://doi.org/10.1016/i.cell.2013.05.039 PMID:23746838

31. Wheeler HE, Kim SK. Genetics and genomics of human ageing. Philos Trans R Soc Lond B Biol Sci. 2011; 366:43-50.

https://doi.org/10.1098/rstb.2010.0259 PMID:21115529

32. Belsky DW, Caspi A, Houts R, Cohen HJ, Corcoran DL, Danese A, Harrington $H$, Israel S, Levine ME, Schaefer JD, Sugden K, Williams B, Yashin Al, et al. Quantification of biological aging in young adults. Proc Natl Acad Sci U S A. 2015; 112:E4104-10. https://doi.org/10.1073/pnas.1506264112 PMID:26150497

33. Blodgett JM, Theou O, Howlett SE, Rockwood K. A frailty index from common clinical and laboratory tests predicts increased risk of death across the life course. Geroscience. 2017; 39:447-55. https://doi.org/10.1007/s11357-017-9993-7 PMID:28866737

34. Cho IH, Park KS, Lim CJ. An empirical comparative study on biological age estimation algorithms with an application of Work Ability Index (WAI). Mech Ageing Dev. 2010; 131:69-78.

https://doi.org/10.1016/i.mad.2009.12.001 PMID:20005245

35. Cruz-Jentoft AJ, Daragjati J, Fratiglioni L, Maggi S, Mangoni AA, Mattace-Raso F, Paccalin M, Polidori MC, Topinkova E, Ferrucci L, Pilotto A, and MPI_AGE Investigators. Using the Multidimensional Prognostic Index (MPI) to improve cost-effectiveness of interventions in multimorbid frail older persons: results and final recommendations from the MPI_AGE European Project. Aging Clin Exp Res. 2020; 32:861-68.

https://doi.org/10.1007/s40520-020-01516-0 PMID: $\underline{32180170}$

36. Klemera P, Doubal S. A new approach to the concept and computation of biological age. Mech Ageing Dev. 2006; 127:240-48.

https://doi.org/10.1016/j.mad.2005.10.004 PMID:16318865

37. Levine ME. Modeling the rate of senescence: can estimated biological age predict mortality more accurately than chronological age? J Gerontol A Biol Sci Med Sci. 2013; 68:667-74.

https://doi.org/10.1093/gerona/gls233

PMID:23213031

38. Mitnitski A, Collerton J, Martin-Ruiz C, Jagger C, von Zglinicki T, Rockwood K, Kirkwood TB. Age-related frailty and its association with biological markers of ageing. BMC Med. 2015; 13:161. https://doi.org/10.1186/s12916-015-0400-x

PMID:26166298

39. Pilotto A, Custodero C, Maggi S, Polidori MC, Veronese N, Ferrucci L. A multidimensional approach to frailty in older people. Ageing Res Rev. 2020; 60:101047.

https://doi.org/10.1016/j.arr.2020.101047

PMID:32171786

40. Baldi P, Brunak S. Bioinformatics: The Machine Learning Approach. 2nd ed. Cambridge, MA, USA: MIT Press; 2001.

41. Putin E, Mamoshina $P$, Aliper A, Korzinkin $M$, Moskalev A, Kolosov A, Ostrovskiy A, Cantor C, Vijg J, Zhavoronkov A. Deep biomarkers of human aging: Application of deep neural networks to biomarker development. Aging (Albany NY). 2016; 8:1021-33. https://doi.org/10.18632/aging.100968 PMID:27191382

42. Bobrov E, Georgievskaya A, Kiselev K, Sevastopolsky A, Zhavoronkov A, Gurov S, Rudakov K, Del Pilar Bonilla Tobar $\mathrm{M}$, Jaspers $\mathrm{S}$, Clemann $\mathrm{S}$. PhotoAgeClock: deep learning algorithms for development of non-invasive visual biomarkers of aging. Aging (Albany NY). 2018; 10:3249-59.

https://doi.org/10.18632/aging.101629 PMID: $\underline{30414596}$

43. Cole JH, Ritchie SJ, Bastin ME, Valdés Hernández MC, Muñoz Maniega S, Royle N, Corley J, Pattie A, Harris SE, Zhang Q, Wray NR, Redmond P, Marioni RE, et al. Brain age predicts mortality. Mol Psychiatry. 2018; 23:1385-92.

https://doi.org/10.1038/mp.2017.62

PMID:28439103

44. Shamir L, Wolkow CA, Goldberg IG. Quantitative measurement of aging using image texture entropy. Bioinformatics. 2009; 25:3060-63.

https://doi.org/10.1093/bioinformatics/btp571 PMID:19808878

45. Fleischer JG, Schulte R, Tsai HH, Tyagi S, Ibarra A, Shokhirev MN, Huang L, Hetzer MW, Navlakha S. Predicting age from the transcriptome of human dermal fibroblasts. Genome Biol. 2018; 19:221. https://doi.org/10.1186/s13059-018-1599-6 PMID: $\underline{30567591}$

46. Johnson AA, Shokhirev MN, Wyss-Coray T, Lehallier B. Systematic review and analysis of human proteomics aging studies unveils a novel proteomic aging clock and identifies key processes that change with age. Ageing Res Rev. 2020; 60:101070.

https://doi.org/10.1016/j.arr.2020.101070

PMID: $\underline{32311500}$ 
47. Tanaka T, Biancotto A, Moaddel R, Moore AZ, Gonzalez-Freire $M$, Aon MA, Candia J, Zhang $P$, Cheung F, Fantoni G, Semba RD, Ferrucci L, and CHI consortium. Plasma proteomic signature of age in healthy humans. Aging Cell. 2018; 17:e12799.

https://doi.org/10.1111/acel.12799

PMID:29992704

48. Pilia G, Chen WM, Scuteri A, Orrú M, Albai G, Dei M, Lai S, Usala G, Lai M, Loi P, Mameli C, Vacca L, Deiana $M$, et al. Heritability of cardiovascular and personality traits in 6,148 Sardinians. PLoS Genet. 2006; 2:e132.

https://doi.org/10.1371/journal.pgen.0020132 PMID:16934002

49. Scuteri A, Najjar SS, Orrú M, Albai G, Strait J, Tarasov KV, Piras MG, Cao A, Schlessinger D, Uda M, Lakatta EG. Age- and gender-specific awareness, treatment, and control of cardiovascular risk factors and subclinical vascular lesions in a founder population: the SardiNIA Study. Nutr Metab Cardiovasc Dis. 2009; 19:532-41.

https://doi.org/10.1016/i.numecd.2008.11.004 PMID:19321325

50. Cesari M, Penninx BW, Pahor M, Lauretani F, Corsi AM, Rhys Williams G, Guralnik JM, Ferrucci L. Inflammatory markers and physical performance in older persons: the InCHIANTI study. J Gerontol A Biol Sci Med Sci. 2004; 59:242-48.

https://doi.org/10.1093/gerona/59.3.m242

PMID: 15031308

51. Ferrucci L, Bandinelli S, Benvenuti E, Di lorio A, Macchi C, Harris TB, Guralnik JM. Subsystems contributing to the decline in ability to walk: bridging the gap between epidemiology and geriatric practice in the InCHIANTI study. J Am Geriatr Soc. 2000; 48:1618-25.

https://doi.org/10.1111/j.1532-5415.2000.tb03873.x PMID:11129752

52. Cousminer DL, Berry DJ, Timpson NJ, Ang W, Thiering E, Byrne EM, Taal HR, Huikari V, Bradfield JP, Kerkhof M, Groen-Blokhuis MM, Kreiner-Møller E, Marinelli $M$, et al, and ReproGen Consortium, and Early Growth Genetics (EGG) Consortium. Genome-wide association and longitudinal analyses reveal genetic loci linking pubertal height growth, pubertal timing and childhood adiposity. Hum Mol Genet. 2013; 22:2735-47.

https://doi.org/10.1093/hmg/ddt104

PMID:23449627

53. Ho TK. Random Decision Forests. Proceedings of 3rd International Conference on Document Analysis and Recognition, 1995; 1:278-282.

https://doi.org/10.1109/ICDAR.1995.598994
54. Zou H, Hastie T. Regularization and variable selection via the elastic net. J R Stat Soc Ser B Stat Methodol. 2005; 67:301-20.

https://doi.org/10.1111/j.1467-9868.2005.00503.x

55. Fukunaga K, Narendra PM. A Branch and Bound Algorithm for Computing k-Nearest Neighbors. IEEE Trans Comput. 1975; C-24:750-53. https://doi.org/10.1109/T-C.1975.224297

56. Tibshirani R. Regression Shrinkage and Selection via the Lasso. J R Stat Soc Ser B Methodol. 1996; 58:267-88. https://doi.org/10.1111/j.2517-6161.1996.tb02080.x

57. Cortes C, Vapnik V. Support-vector networks. Mach Learn. 1995; 20:273-97. http://dx.doi.org/10.1007/BF00994018

58. Pedregosa F, Varoquaux G, Gramfort A, Michel V, Thirion B, Grisel O, Blondel M, Prettenhofer $P$, Weiss R, Dubourg V, Vanderplas J, Passos A, Cournapeau D, et al. Scikit-learn: Machine Learning in Python. J Mach Learn Res. 2011; 12:2825-30.

59. Jaakkola T, Haussler D. Exploiting Generative Models in Discriminative Classifiers. Adv Neural Inf Process. 1999; 7.

60. Deelen J, Uh HW, Monajemi R, van Heemst D, Thijssen $P E$, Böhringer $S$, van den Akker EB, de Craen AJ, Rivadeneira F, Uitterlinden AG, Westendorp RG, Goeman JJ, Slagboom PE, et al. Gene set analysis of GWAS data for human longevity highlights the relevance of the insulin/IGF-1 signaling and telomere maintenance pathways. Age (Dordr). 2013; 35:235-49. https://doi.org/10.1007/s11357-011-9340-3 PMID:22113349

61. Jeck WR, Siebold AP, Sharpless NE. Review: a metaanalysis of GWAS and age-associated diseases. Aging Cell. 2012; 11:727-31. https://doi.org/10.1111/j.1474-9726.2012.00871.x PMID:22888763

62. Lu AT, Xue L, Salfati EL, Chen BH, Ferrucci L, Levy D, Joehanes R, Murabito JM, Kiel DP, Tsai PC, Yet I, Bell $\mathrm{JT}$, Mangino $\mathrm{M}$, et al. GWAS of epigenetic aging rates in blood reveals a critical role for TERT. Nat Commun. 2018; 9:387.

https://doi.org/10.1038/s41467-017-02697-5 PMID:29374233

63. Archer KJ, Kimes RV. Empirical characterization of random forest variable importance measures. Comput Stat Data Anal. 2008; 52:2249-60. https://doi.org/10.1016/j.csda.2007.08.015

64. Costa PT, McCrae RR, Kay G. Persons, Places, and Personality: Career Assessment Using the Revised NEO Personality Inventory. J Career Assess. 1995; 3:123-39. https://doi.org/10.1177/106907279500300202 
65. McCrory C, Fiorito G, Hernandez B, Polidoro S, O'Halloran AM, Hever A, Ni Cheallaigh C, Lu AT, Horvath S, Vineis P, Kenny RA. GrimAge Outperforms Other Epigenetic Clocks in the Prediction of AgeRelated Clinical Phenotypes and All-Cause Mortality. J Gerontol A Biol Sci Med Sci. 2021; 76:741-49.

https://doi.org/10.1093/gerona/glaa286

PMID: 33211845

66. Marioni RE, Suderman $M$, Chen $B H$, Horvath $S$, Bandinelli S, Morris T, Beck S, Ferrucci L, Pedersen NL, Relton CL, Deary IJ, Hägg S. Tracking the Epigenetic Clock Across the Human Life Course: A Meta-analysis of Longitudinal Cohort Data. J Gerontol A Biol Sci Med Sci. 2019; 74:57-61.

https://doi.org/10.1093/gerona/gly060

PMID:29718110

67. Sun ED, Michaels TCT, Mahadevan L. Optimal control of aging in complex networks. Proc Natl Acad Sci U S A. 2020; 117:20404-10.

https://doi.org/10.1073/pnas.2006375117 PMID:32817469

68. Vural DC, Morrison G, Mahadevan L. Aging in complex interdependency networks. Phys Rev E Stat Nonlin Soft Matter Phys. 2014; 89:022811.

https://doi.org/10.1103/PhysRevE.89.022811

PMID:25353538

69. Stroustrup N, Anthony WE, Nash ZM, Gowda V, Gomez A, López-Moyado IF, Apfeld J, Fontana W. The temporal scaling of Caenorhabditis elegans ageing. Nature. 2016; 530:103-07.

https://doi.org/10.1038/nature16550

PMID:26814965

70. Heidenreich PA, Trogdon JG, Khavjou OA, Butler J, Dracup K, Ezekowitz MD, Finkelstein EA, Hong $Y$, Johnston SC, Khera A, Lloyd-Jones DM, Nelson SA, Nichol G, et al, and American Heart Association Advocacy Coordinating Committee, and Stroke Council, and Council on Cardiovascular Radiology and Intervention, and Council on Clinical Cardiology, and Council on Epidemiology and Prevention, and Council on Arteriosclerosis, and Thrombosis and Vascular Biology, and Council on Cardiopulmonary, and Critical Care, and Perioperative and Resuscitation, and Council on Cardiovascular Nursing, and Council on the Kidney in Cardiovascular Disease, and Council on Cardiovascular Surgery and Anesthesia, and Interdisciplinary Council on Quality of Care and Outcomes Research. Forecasting the future of cardiovascular disease in the United States: a policy statement from the American Heart Association. Circulation. 2011; 123:933-44.

https://doi.org/10.1161/CIR.0b013e31820a55f5 PMID:21262990

71. Kaplan DT, Furman MI, Pincus SM, Ryan SM, Lipsitz
LA, Goldberger AL. Aging and the complexity of cardiovascular dynamics. Biophys J. 1991; 59:945-49. https://doi.org/10.1016/S0006-3495(91)82309-8 PMID:2065195

72. Volpato S, Guralnik JM, Ferrucci L, Balfour J, Chaves P, Fried LP, Harris TB. Cardiovascular disease, interleukin-6, and risk of mortality in older women: the women's health and aging study. Circulation. 2001; 103:947-53. https://doi.org/10.1161/01.cir.103.7.947 PMID: 11181468

73. Pyrkov TV, Slipensky K, Barg M, Kondrashin A, Zhurov B, Zenin A, Pyatnitskiy M, Menshikov L, Markov S, Fedichev PO. Extracting biological age from biomedical data via deep learning: too much of a good thing? Sci Rep. 2018; 8:5210. https://doi.org/10.1038/s41598-018-23534-9 PMID:29581467

74. Baracho GV, Nudelman V, Isaac L. Molecular characterization of homozygous hereditary factor I deficiency. Clin Exp Immunol. 2003; 131:280-86. https://doi.org/10.1046/i.1365-2249.2003.02077.x PMID:12562389

75. Fagerness JA, Maller JB, Neale BM, Reynolds RC, Daly MJ, Seddon JM. Variation near complement factor I is associated with risk of advanced AMD. Eur J Hum Genet. 2009; 17:100-04.

https://doi.org/10.1038/ejhg.2008.140

PMID:18685559

76. Minta JO, Fung M, Paramaswara B. Transcriptional and post-transcriptional regulation of complement factor I (CFI) gene expression in Hep G2 cells by interleukin-6. Biochim Biophys Acta. 1998; 1442:286-95.

https://doi.org/10.1016/s0167-4781(98)00189-4 PMID:9804975

77. Anderson DH, Radeke MJ, Gallo NB, Chapin EA, Johnson PT, Curletti CR, Hancox LS, Hu J, Ebright JN, Malek G, Hauser MA, Rickman CB, Bok D, et al. The pivotal role of the complement system in aging and age-related macular degeneration: hypothesis revisited. Prog Retin Eye Res. 2010; 29:95-112. https://doi.org/10.1016/i.preteyeres.2009.11.003 PMID: 19961953

78. Kavanagh D, Yu Y, Schramm EC, Triebwasser M, Wagner EK, Raychaudhuri S, Daly MJ, Atkinson JP, Seddon JM. Rare genetic variants in the CFI gene are associated with advanced age-related macular degeneration and commonly result in reduced serum factor I levels. Hum Mol Genet. 2015; 24:3861-70. https://doi.org/10.1093/hmg/ddv091 PMID:25788521 
79. Seddon JM, Yu Y, Miller EC, Reynolds R, Tan PL, Gowrisankar S, Goldstein JI, Triebwasser M, Anderson HE, Zerbib J, Kavanagh D, Souied E, Katsanis N, et al. Rare variants in CFI, C3 and C9 are associated with high risk of advanced age-related macular degeneration. Nat Genet. 2013; 45:1366-70.

https://doi.org/10.1038/ng.2741

PMID:24036952

80. van de Ven JP, Nilsson SC, Tan PL, Buitendijk GH, Ristau T, Mohlin FC, Nabuurs SB, Schoenmaker-Koller FE, Smailhodzic D, Campochiaro PA, Zack DJ, Duvvari $\mathrm{MR}$, Bakker B, et al. A functional variant in the CFI gene confers a high risk of age-related macular degeneration. Nat Genet. 2013; 45:813-17.

https://doi.org/10.1038/ng.2640

PMID:23685748

81. Grumach AS, Leitão MF, Arruk VG, Kirschfink M, Condino-Neto A. Recurrent infections in partial complement factor I deficiency: evaluation of three generations of a Brazilian family. Clin Exp Immunol. 2006; 143:297-304.

https://doi.org/10.1111/i.1365-2249.2005.02988.x PMID:16412054

82. Markiewski MM, Lambris JD. The role of complement in inflammatory diseases from behind the scenes into the spotlight. Am J Pathol. 2007; 171:715-27. https://doi.org/10.2353/ajpath.2007.070166 PMID:17640961

83. Schmidt JC, Cech TR. Human telomerase: biogenesis, trafficking, recruitment, and activation. Genes Dev. 2015; 29:1095-105.

https://doi.org/10.1101/gad.263863.115 PMID:26063571

84. van de Vegte YJ, Tegegne BS, Verweij N, Snieder H, van der Harst P. Genetics and the heart rate response to exercise. Cell Mol Life Sci. 2019; 76:2391-409. https://doi.org/10.1007/s00018-019-03079-4 PMID: $\underline{30919020}$

85. Blackburn EH, Epel ES, Lin J. Human telomere biology: A contributory and interactive factor in aging, disease risks, and protection. Science. 2015; 350:1193-98. https://doi.org/10.1126/science.aab3389 PMID:26785477

86. Hewitt G, Jurk D, Marques FD, Correia-Melo C, Hardy T, Gackowska A, Anderson R, Taschuk M, Mann J, Passos JF. Telomeres are favoured targets of a persistent DNA damage response in ageing and stressinduced senescence. Nat Commun. 2012; 3:708. https://doi.org/10.1038/ncomms1708 PMID:22426229

87. GTEx Consortium. The Genotype-Tissue Expression (GTEx) project. Nat Genet. 2013; 45:580-85. https://doi.org/10.1038/ng.2653

PMID:23715323

88. He L, Kernogitski Y, Kulminskaya I, Loika Y, Arbeev KG, Loiko E, Bagley O, Duan M, Yashkin A, Ukraintseva SV, Kovtun M, Yashin Al, Kulminski AM. Corrigendum: Pleiotropic Meta-Analyses of Longitudinal Studies Discover Novel Genetic Variants Associated with AgeRelated Diseases. Front Genet. 2017; 8:226. https://doi.org/10.3389/fgene.2017.00226 PMID:29375618

89. Grammatikakis I, Panda AC, Abdelmohsen K, Gorospe M. Long noncoding RNAs(IncRNAs) and the molecular hallmarks of aging. Aging (Albany NY). 2014; 6:9921009.

https://doi.org/10.18632/aging.100710 PMID:25543668

90. Greco S, Gorospe M, Martelli F. Noncoding RNA in age-related cardiovascular diseases. J Mol Cell Cardiol. 2015; 83:142-55.

https://doi.org/10.1016/i.yimcc.2015.01.011 PMID:25640162

91. Kim C, Kang D, Lee EK, Lee JS. Long Noncoding RNAs and RNA-Binding Proteins in Oxidative Stress, Cellular Senescence, and Age-Related Diseases. Oxidative Medicine and Cellular Longevity. 2017. https://www.hindawi.com/journals/omcl/2017/2062 384/abs/

92. Kour S, Rath PC. Long noncoding RNAs in aging and age-related diseases. Ageing Res Rev. 2016; 26:1-21. https://doi.org/10.1016/j.arr.2015.12.001 PMID:26655093

93. Chen T, Guestrin C. XGBoost: A Scalable Tree Boosting System. KDD '16: Proceedings of the 22nd ACM SIGKDD International Conference on Knowledge Discovery and Data Mining. 2016; 785-794 https://doi.org/10.1145/2939672.2939785

94. Glass D, Viñuela A, Davies MN, Ramasamy A, Parts L, Knowles D, Brown AA, Hedman AK, Small KS, Buil A, Grundberg E, Nica AC, Di Meglio P, et al, and UK Brain Expression consortium, and MUTHER consortium. Gene expression changes with age in skin, adipose tissue, blood and brain. Genome Biol. 2013; 14:R75. https://doi.org/10.1186/gb-2013-14-7-r75 PMID:23889843

95. Peters MJ, Joehanes R, Pilling LC, Schurmann C, Conneely KN, Powell J, Reinmaa E, Sutphin GL, Zhernakova A, Schramm K, Wilson YA, Kobes S, Tukiainen $\mathrm{T}$, et al, and NABEC/UKBEC Consortium. The transcriptional landscape of age in human peripheral blood. Nat Commun. 2015; 6:8570. https://doi.org/10.1038/ncomms9570 PMID:26490707 


\section{SUPPLEMENTARY MATERIALS}

\section{Supplementary Methods}

\section{Description of data cleaning and binning procedures}

\section{Dataset cleaning strategy}

Both SardiNIA and InCHIANTI datasets contained substantial portions of missing values. Traits and samples were dropped iteratively to preserve maximum data size while maintaining a complete set of values. In wave 1 of SardiNIA, the cleaning method reduced the number of participants from 6165 to 4817 and the number of traits from 183 to 148 .

\section{Binning strategy}

Binning strategies were determined empirically to maximize both model performance and data retention. We abbreviated a binning strategy as a tuple (bin size, youngest age, oldest age). In the SardiNIA dataset, optimal binning for wave 1 was set at $(5,12,77)$, which allowed for 120 training samples and 13 testing samples from each bin per split and 13 total age bins (1560 training and 169 test samples in total). For wave 2, bins with $(5,16,81)$ were determined and yielded 83 training and nine testing samples per age bin with 13 total bins (1079 training and 117 test samples in total). For wave 3 , bins with $(5,20,75)$ were determined and yielded 81 training and eight testing samples per age bin with 12 total bins (984 training and 96 test samples in total). For wave 4, bins with $(5,21,81)$ were determined and yielded 49 training and five testing samples for each of 12 age bins (588 training and 60 testing samples in total). A schematic of this binning is shown in Supplementary Figure 2.

We used different binning strategies for the commontrait framework in SardiNIA to maximize sample representation. The same age ranges and bin sizes (5 years) were used to make fair comparisons to the full-trait model. Sample sizes were increased to saturate the smallest bin size: wave 1 (160 training, 18 testing), wave 2 (107 training, 12 testing), wave 3 (213 training, 24 testing), wave 4 (64 training, seven testing).

As a study of frailty in older individuals, InCHIANTI contained much lower representation of younger age groups. Despite this, the same binning strategy was employed to maintain fair comparisons to SardiNIA. Interestingly, the smaller sample sizes did not substantially decrease model performance. In the baseline study (wave 0$)$, bins of $(6,23,89)$ provided sufficient data for training (19) and testing (2) for each of 11 age bins (209 training and 22 testing samples in total). For wave 1 , we used $(6,26,86)$ which yielded 19 training and two testing samples for each of 10 age bins (190 training and 20 testing in total). For wave 2, we used $(6,27,87)$ which yielded 19 training and two testing samples for each of 10 age bins (190 training and 20 testing in total). For wave 3 , we used $(6,31,91)$ which yielded 20 training and two testing samples for each of 10 age bins (200 training and 20 testing in total).

\section{Machine learning model selection}

\section{Description of main machine learning models investigated in the study}

In addition to the random forest classifier, we explored other classical machine learning models outlined below.

\section{Elastic net}

We utilized the Scikit-Learn implementation of elastic net regression (ElasticNet) [58], which was equivalent to the glmnet implementation in $\mathrm{R}$ with "alpha" and "11_ratio" in Scikit-Learn corresponding to "lambda" and "alpha" in glmnet. Elastic net uses a penalty defined by a linear combination of the L1 and L2 penalties of the LASSO and Ridge regressions. In our model, we used the default uniform penalty weights specified by " 11 ratio $=0.5$ " and "alpha $=1.0$ ".

\section{K-nearest neighbors}

We implemented the k-nearest neighbors regressor from the Scikit-Learn library [58]. The algorithm predicts the target variable from local interpolation of the $\mathrm{k}$ nearest neighboring data points in highdimensional trait space. Our model used "n_neighbors $=20 "$, "weights = 'distance"", " $p=1 "$, which were determined heuristically.

\section{Support vector machine (SVR)}

We implemented the epsilon-support vector regressor (SVR) from the Scikit-Learn library [58]. Our model used the default parameters of " $\mathrm{C}=1.0$ " and "epsilon $=0.1$ ".

Multiple linear regression (LinReg)

We implemented the default multiple linear regression model (LinearRegression) from the Scikit-Learn library [58].

Lasso

Lasso regression was implemented from the ScikitLearn library [58]. We used the elastic net model (ElasticNet) with "11_ratio $=1.0$ " to recover the lasso regression. 


\section{Saturation of model performance from traits and samples}

Model performance measured with $\mathrm{R}^{2}$ saturated after an intermediate number of traits and after an intermediate number of individuals (i.e., the saturation in performance occurred before the maximum number of traits or individuals was reached in virtually all cases). For example, increasing the number of traits resulted in marginal $\mathrm{R}^{2}$ gains after $\sim 30$ traits and after $\sim 80$ individuals per bin in the RFC full-trait model for the SardiNIA baseline study (Supplementary Figure 3). Corroborating these results, we observed no significant difference in model performance $\left(\mathrm{R}^{2}\right)$ when the model was trained on the entire data as compared to uniform sampling from age bins (data not shown).

Trait transformation with linear discriminant analysis (LDA) prior to training/testing increased predictive performance in most machine learning models (Supplementary Figure 3).

\section{Physiological age and physiological aging rate (PAR) computed with different model frameworks}

\section{Random forest classifier (RFC) model for follow-up waves \\ Model performances for the three follow-up studies (W2, W3, W4) were highly similar to that of the baseline (W1) SardiNIA study (see Supplementary Figure 4). Physiological ages were well-correlated with chronological age, and the PAR values were approximately uniformly distributed and weakly correlated with chronological age. It should be noted that model performance $\left(\mathrm{R}^{2}\right)$ decreased for the later follow-up studies, which was likely due to a reduced number of participants and reduced overlap with the original trait measurements in the $\mathrm{W} 1$.}

\section{InCHIANTI-trained random forest classifer model (RFC)}

We replicated our findings in the InCHIANTI longitudinal study. The same RFC machine learning framework yielded comparably well-correlated physiological age measurements as those from the SardiNIA study (see Supplementary Figure 5). Likewise, the distribution of PAR measurements was roughly uniform with age across all waves of the InCHIANTI study.

\section{Similar distribution of PARs observed in SardiNIA and InCHIANTI studies}

Despite training the RFC models on studies with different sets of quantitative traits, the PARs of individuals in the SardiNIA study and the PARs of individuals in the InCHIANTI were distributed similarly (see Supplementary Figure 7A). Furthermore, most of the difference in PAR distribution between the two studies are explained by the oldest and youngest age bins. By removing PAR predictions for these edge bins, the PAR distributions are nearly identical for SardiNIA and InCHIANTI (Supplementary Figure 7B).

Common-trait model (RFC) trained on SardiNIA data Reducing the traits to a subset of common clinical and cardiovascular measurements from the SardiNIA study (see Supplementary Table 1 for the description of common traits) resulted in comparable model performance as in the full-trait model. Physiological ages were well-correlated with chronological age, and PARs were weakly correlated with chronological age across all SardiNIA studies. Similarly, the later follow-up waves observed lower model performance, possibly due to decreased numbers of participants and overlapping traits. The PARs obtained from the full-trait model and the common-trait model were highly correlated $\left(\mathrm{R}^{2}=0.798\right.$; see Supplementary Figure 9).

Elastic net regression model trained on SardiNIA data A high-performing regression method was the elastic net regressor $\left(\mathrm{R}^{2}=0.84\right.$ for SardiNIA W1). Previous studies have used elastic net regression for age estimation from blood biochemical measurements [41] and for DNAm age calculation $[1,20]$. Using the elastic net model in lieu of the random forest model yielded comparable results across each baseline and follow-up study of SardiNIA (data not shown).

\section{Gender-separated analysis of PARs}

To determine if physiological differences between the sexes biased the predictive models, we analyzed the PAR measurements from the RFC model developed on data including both genders to determine any differences in the mean or spread of the PARs. We observed no significant difference in the physiological aging rate (PAR) and physiological age acceleration (PAA) between male and female study participants (Supplementary Figure 6).

\section{Top traits in SardiNIA and InCHIANTI}

\section{Top traits for the RFC model in InCHIANTI study}

The top traits in the InCHIANTI study were obtained using baseline data with the same two scoring strategies. Several cardiovascular traits were highly ranked including pulse wave velocity (VEL), systolic blood pressured (23_V28), proximal amplitude (APROX), repolarization phase (RIPOL), and atrioventricular conduction time (TAV) among others; which reflected the high ranking of cardiovascular traits in the SardiNIA data. Another 
top trait across multiple ranking methods was creatinine clearance (CLCR, UCRE24), which is an important indicator of renal health (Cockcroft \& Gault, 1976) and has been associated with aging (Anderson \& Brenner, 1986). Creatinine levels were also highly ranked in SardiNIA. Additional overlaps between top 20\% InCHIANTI and the highestranking SardiNIA traits include waist circumference (VITA), blood nitrogen levels (BUN), blood fibrinogen levels (FIBRIN), cholesterol levels (COLTOT, COLLDL, OX_LDL), sodium intake (VN26), hypertension (IPERT1), uric acid (URICO), transferrin (STRMG), IL-6 (IL_6). Other top traits corresponded to frailty markers such as general frailty (FRAIL, ALLFRA, NALLFR), exhaustion (FEXHAU), weight loss (FWGTLS), grip strength (FSTRNC), visual acuity (24_V35), and gait speed (WLK1A, WSPD1B, WLK1MN, 17_V2, FWLKCT)many of which have been linked to aging (Fulop et al., 2010; Kan et al., 2010; Bohannon et al., 2008). Other high-ranking traits from the three scoring methods included diagnosis of dementia (VASDEM, DEMENT), insulin-like growth factor I (TIGF), fatty acid levels (TFA_MO, C24_1B), coordination (PEGONE, 25_V14, $22 \_$V34), and a variety of coordination task and questionnaire results $\left(18 \_\mathrm{V} 4,18 \_\mathrm{V} 8,25 \_\mathrm{V} 24,25 \_\mathrm{V} 13\right.$, 4_V75, 5_V26, 1_N8, 17_V2, 8_V1, 12_V1, Q0208, etc). Refer to Supplementary Figure 16.

\section{Top traits for the common-trait RFC model in SardiNIA}

The top traits among the common clinical and cardiovascular SardiNIA traits were determined using the three scoring methods outlined in the Methods section. For these methods, most of the top traits from the full-trait set were present. The overlapping traits included pulse wave velocity (pwv), CCA intima media thickness (vasIMT), other cardiovascular traits (vasEDV, vasvti, vasPSV, vasIP, vasDiaDiam, etc), and waist circumference (exmWaist). Additional overlaps included various blood serum levels of sodium (labsSodiedemia), uric acid (labsAcidourico), and alanine aminotransferase (labsALT). A notable difference in the top traits for the common-trait model was the lack of NEOPIR personality traits (o1, e5, a4, form), which were not present in the common-trait set. There was high rank-correlation between the commontrait and full-trait sets (Spearman correlation, $\rho=0.92$ ). Refer to Supplementary Figure 15.

\section{Reproducibility of PAR measurements across follow-up studies}

\section{RFC model in SardiNIA study}

We determined the reproducibility of individual PARs across time by using the RFC model to measure PARs across all four waves of the SardiNIA study. The correlation between the PARs across each consecutive wave ( $\Delta t=3-4$ years) was relatively stable (e.g., $\mathrm{R}^{2} \sim$ 0.4 between the first two waves, see Supplementary Figure 18) but decayed over longer time periods (e.g., $\mathrm{R}^{2}$ $\sim 0.3$ for $\Delta t=6-7$ years, $\mathrm{R}^{2} \sim 0.2$ for $\Delta t=9-10$ years). PARs derived from the common-trait model observed the same trends with marginally higher temporal stability (see following subsection). Notably, the InCHIANTI PARs showed higher consistency over time than those from SardiNIA $\left(\mathrm{R}^{2}=0.55\right.$ between baseline and follow-up \#1, $\mathrm{R}^{2}=0.52$ between baseline and follow-up \#2, $R^{2}=0.46$ between baseline and follow-up \#3, see following subsection). These results indicated that the physiological aging rate was stable for an individual in the short-term - from several years up to a decade - but appreciably destabilized over longer periods of time. This destabilization may be reflecting the environmental influences and heterogeneities. PAR trajectories showcased the generally observed trend in which slow agers retained low PARs while fast agers retained high PARs across time (see Supplementary Figure 18A, 18B). In fact, the average standard deviation between PARs for a given individual across the four waves was around 0.1 for both the full-trait model and the common-trait model.

\section{Common-trait RFC model in SardiNIA study}

PAR measurements obtained using the common-trait model for the same individual were correlated across each of the baseline and follow-up studies of SardiNIA (see Supplementary Figure 18C, 18D) such that there was notable stability of PAR estimates over the period of a few years. The mean standard deviation of individual PAR measurements across the four waves of the study was 0.085 . The correlation between PARs decreased monotonically with increased time $(\Delta t)$ between the measurements, which suggested that environmental influences can noticeably change the PAR after the span of a few years. Alternatively, this destabilization in the PAR values over longer time periods may represent an artefact of changes in data collection procedures and trait measurements in the longitudinal studies.

\section{RFC model in InCHIANTI study}

PAR measurements obtained from the RFC model for the same individual were highly correlated across each of the baseline and follow-up studies of InCHIANTI (see Supplementary Figure 18E, 18F) such that there was notable stability of PAR estimates over the period of a few years. The mean standard deviation of individual PAR measurements across the four waves of the study was 0.048. The correlation between PARs appeared to decrease with increased time $(\Delta t)$ between the measurements, which corroborated similar observations made in the SardiNIA models. 


\section{Alternative age acceleration metric}

\section{Physiological age acceleration (PAA)}

The physiological age acceleration (PAA) corresponds to the difference between the physiological age and chronological age of an individual and was calculated as

$$
P A A=\text { Physiological Age }- \text { Chronological Age }
$$

The PAA is similar to the PAR in that it measures the progression of an individual's aging trajectory relative to other individuals with the same chronological age. This measurement was most comparable to DNAm age acceleration.

\section{Epigenetic age acceleration (EAA)}

The epigenetic age acceleration was similarly calculated as the difference between the predicted epigenetic age and the chronological age:

$$
E A A=\text { Epigenetic Age }- \text { Chronological Age }
$$

Our measurement of epigenetic age acceleration was identical to previous methods of calculating intrinsic epigenetic age acceleration [62].

\section{Controlling for sex and age in the comparison of PAR and EAR}

In the main text, we presented the correlation between PAR and EAR without adjusting for any co-variation by sex or chronological age (see Figure 3 ). Since both aging rates are likely to be at least partially dependent on chronological age (and sex to an extent), we also compared the residual PAR and EAR values after regressing out sex and chronological age using an ordinary least-squares linear model. The correlation between PAR and EAR was reduced but still positive $\left(R^{2}=0.182\right.$; see Supplementary Figure 12$)$.

\section{Linear rescaling and trimming to correct for the imbalanced distribution of PARs across age}

\section{Motivation for linearly rescaled predicted ages}

The random forest classifier showcased a slight deviation from a uniform distribution of PAR measurements despite the high predictive performance $\left(\mathrm{R}^{2}=0.86\right.$ between physiological age and chronological age). Due to the deviation, the oldest individuals recorded mean PAR $<1$ and the youngest individuals had mean PAR > 1 . The deviation may be representative of selection bias from the assumedly longer survival of slower aging (PAR $<1)$ individuals. However, another possible source of this deviation was the lack of data for individuals beyond the age range specified in SardiNIA. The elastic net model, a regression method, produced a notably milder deviation. To reduce the deviation, we attempted to linearly rescale the predicted ages into the same dimensions as chronological age and enforce a slope of $\sim 1.0$ between the rescaled physiological age and the chronological age (see Supplementary Figure 14A). The rescaling preserved the relative information obtained from the predictive model and reduced age-associated imbalances in the PAR. The rescaling resulted in normal distributions of PARs centered around a mean age value corresponding to the chronological age of that group (see Supplementary Figure 14B). The equation for linear rescaling was

$$
[\text { Physiological Age }]=\frac{[\text { Predicted Age }]-\beta}{\alpha},
$$

where $\alpha$ was the slope of the linear least-squares regression with an L2 norm on coordinate pairs determined by (chronological age, predicted age), and $\beta$ was the corresponding intercept. The L2 norm was used since we expected the physiological ages to be normally distributed around the chronological age for all individuals of that given chronological age.

\section{Analytic details for the linear rescaling of physiological age}

A linear rescaling was applied to the predicted ages to obtain the physiological age measurements. The rescaling enforced the mean of the physiological ages as the chronological age corresponding to each age group and therefore symmetrized the distribution of the eRAs across all ages. This was achieved with:

$$
[\text { Physiological Age }]=\frac{[\text { Predicted Age }]-\beta}{\alpha}
$$

Where $\alpha$ was the slope of the linear least-squares regression with an L2 norm on coordinate pairs determined by (chronological age, predicted age), and $\beta$ was the corresponding intercept. The L2 norm was used since we expect the physiological ages to be normally distributed around the chronological age for all individuals of that given chronological age.

In this section, we provide an informal sketch for why the rescaling equation results in the intended centering of the physiological ages around the chronological age. We denote the chronological age as $x$ and the predicted (unscaled) age as $y_{1}$. Using a linear least squares regression with an L2 norm, we obtain the linear model parameters $\alpha$ and $\beta$ such that the line that minimizes the L2 residual is defined as:

$$
y_{1}^{l s}=\alpha x+\beta
$$

We apply the rescaling transformation to obtain the physiological (rescaled) age denoted $y_{2}$. Similarly, we can evaluate the linear least squares regression on the rescaled data to obtain linear model parameters that minimize the L2 norm such that: 


$$
\begin{aligned}
\alpha_{2}, \beta_{2} & =\underset{\alpha_{2}, \beta_{2}}{\operatorname{argmin}} \sum_{i}\left|y_{2, i}^{l s}-y_{2, i}\right|^{2} \\
y_{2, i}^{l s} & =\alpha_{2} x+\beta_{2}
\end{aligned}
$$

Substituting the definition of $y_{2, i}^{l s}$ into the objective function yields:

$$
\alpha_{2}, \beta_{2}=\underset{\alpha_{2}, \beta_{2}}{\operatorname{argmin}} \sum_{i}\left|\alpha_{2} x+\beta_{2}-y_{2, i}\right|^{2}
$$

Since $y_{2, i}$ is the rescaled predicted age, then it must be that $y_{2, i}=\frac{y_{1, i}-\beta}{\alpha}$. Additionally, we can rewrite $x=\frac{y_{1}^{l s}-\beta}{\alpha}$.

Substituting in these two relations yields:

$$
\begin{aligned}
\alpha_{2}, \beta_{2} & =\underset{\alpha_{2}, \beta_{2}}{\operatorname{argmin}} \sum_{i}\left|\alpha_{2} \frac{y_{1}^{l s}-\beta}{\alpha}+\beta_{2}-\frac{y_{1, i}-\beta}{\alpha}\right|^{2} \\
& =\underset{\alpha_{2}, \beta_{2}}{\operatorname{argmin}} \sum_{i}\left|\frac{1}{\alpha}\left(\alpha_{2} y_{1}^{l s}-y_{1, i}\right)-\frac{\beta}{\alpha}\left(\alpha_{2}-1\right)+\beta_{2}\right|^{2}
\end{aligned}
$$

This is minimized for values of $\alpha_{2}=1, \beta_{2}=0$ where it reduces the physiological age objective function to

$$
\sum_{i}\left|\frac{y_{1}^{l s}-y_{1, i}}{\alpha}\right|^{2}=\frac{1}{\alpha^{2}} \sum_{i}\left|y_{1}^{l s}-y_{1, i}\right|^{2}
$$

which is the minimal objective value for the predicted (unscaled) ages. As a result, the least squares linear regression for the rescaled physiological ages can be approximated as a rescaling of the linear regression for the unscaled predicted ages.

\section{Description of trimmed PAR values}

We implemented another approach to reduce the ageassociated imbalance in PAR measurements, which was to include the youngest and oldest edge bins (first five years and last five years for SardiNIA W1) in model training but exclude them from downstream analyses. We refer to the reduced set of measurements as the "trimmed" physiological age and "trimmed" physiological aging rate. Trimming increased the slope of the linear regression between chronological age and physiological age.

\section{Additional details on GWAS significant loci}

APLF ( $p=8.59 \mathrm{E}-8)$ encodes a histone chaperone protein that is involved in non-homologous end-joining (NHEJ) repair of DNA double strand breaks [96, 97], which is linked to aging and age-related disease [98]. ARHGAP15 is associated with diverticulitis [99] and colorectal cancer [100], and was selectively upregulated with age $(r=0.294)$ in colon tissue according to GTEx RNA-seq gene expression profiles. ANKRD26 $(p=2.96 \mathrm{E}-7)$ has been associated with human diabetes mellitus, cardiovascular disease, and neurodegenerative disease in previous genome-wide studies [88] and has been shown to promote diabetes and obesity in mouse models [101, 102]. ANKRD26 is flanked by the LINC00202 locus (see Figure 4A), but analysis with GTEx [87] did not identify any of the significant LINC00202 SNPs as eQTLs for ANKRD26. ZNF518B $(p=3.26 \mathrm{E}-7)$ has been associated with gout [103], colorectal tumor invasion [104, 105], age-related epigenetic changes [106], and is involved in histone modification [107]. Notably, ZNF518B is downexpressed with age in all non-brain GTEx tissues examined including heart $(\mathrm{r}=-0.231)$, liver $(\mathrm{r}=$ $-0.475)$, lung $(r=-0.294)$, thyroid $(r=-0.405)$, and colon $(\mathrm{r}=-0.250)$ (see Supplementary Materials). CSMD1 ( $p=3.96 \mathrm{E}-7)$ was previously associated with familial Parkinson's disease [108] and cognitive function [109]. Accordingly, CSMD1 was downexpressed with age $(r=-0.314)$ in GTEx cerebellum samples and did not appear to be age-associated in the non-brain tissues examined. Using GTEx data, we generated plots of the normalized gene expression value across age for several key genes of interest (see Supplementary Figure 19). Normalized gene expression value was computed as the sum-normalized value for all gene expression values in a given sample/patient (similarly to TPM calculations).

\section{Common-trait physiological aging rates predict mortality}

To determine whether common-trait PARs predicted mortality and lifespan, we performed a random one-toone age-matched comparison on the 329 deceased participants and the remaining living participants in the SardiNIA study. The difference in the mean PAR measurements of the two groups was calculated as $\triangle \mathrm{PAR}$ $=\mathrm{PAR}_{\text {deceased }}-\mathrm{PAR}_{\text {living }}$, and a corresponding $p$-value was obtained from a one-sided $(\triangle \mathrm{PAR}>0)$ one-sample $t$-test. The age-matched grouping was performed 10000 times and $\triangle \mathrm{PAR}$ and $p$-values. The fraction of significantly different $(p<0.05)$ mean PAR values between the deceased and living groups was $65.7 \%$ and the mean $\triangle \mathrm{PAR}$ was 0.013 (see Supplementary Figure 11). More than $99 \%$ of the 10000 random age-matched comparisons reported $\triangle \mathrm{PAR}>0$ as compared to randomized controls where $50.8 \%$ of the comparisons between two randomly age-matched groups had $\triangle \mathrm{PAR}>0$ (Supplementary Figure 11). Estimated lifespans were similarly negatively correlated with PARs in the common-trait model $(r=$ -0.469 , Supplementary Figure 11).

\section{Computational pipeline and code repository}

The computational pipelines can be accessed at the Github code repository: https://github.com/sunericd/SardiNIAgeRates. The repository contains two Python scripts ("runModel.py" and "AgeRatesTurnkey.py") that are necessary for 
running the machine learning framework. The model parameters are specified in the "run_spec.txt" file. In the working directory, the machine learning framework can be run with the command line python runModel.py. After successfully running the model, results will be saved as image files and a tab-separated text file with columns corresponding to [ID] [Age] [Predicted Age] [Physiological Aging Rate] and the rows corresponding to each individual in the SardiNIA/InCHIANTI datasets.

The Github repository also contains two Jupyter notebooks ("results_and_analyses.ipynb" and "top_traits.ipynb") that outline code blocks for generating most of the figures included in the main text and supplementary materials and other examples for analyzing the results of the machine learning framework. Additional Jupyter notebooks in the "Miscellaneous" directory on the repository contain code used to test and develop the predictive frameworks outlined in this investigation.

The computational model was written in Python 3.5 and requires NumPy, SciPy, Matplotlib, Pandas, and Scikitlearn to be installed. Optionally, XGBoost can be installed.

\section{REFERENCES}

96. Mehrotra PV, Ahel D, Ryan DP, Weston R, Wiechens $\mathrm{N}$, Kraehenbuehl R, Owen-Hughes T, Ahel I. DNA repair factor APLF is a histone chaperone. Mol Cell. 2011; 41:46-55.

https://doi.org/10.1016/j.molcel.2010.12.008

PMID:21211722

97. Rulten SL, Fisher $A E$, Robert I, Zuma MC, Rouleau M, Ju L, Poirier G, Reina-San-Martin B, Caldecott KW. PARP-3 and APLF function together to accelerate nonhomologous end-joining. Mol Cell. 2011; 41:33-45.

https://doi.org/10.1016/j.molcel.2010.12.006

PMID:21211721

98. Lombard DB, Chua KF, Mostoslavsky R, Franco S, Gostissa M, Alt FW. DNA repair, genome stability, and aging. Cell. 2005; 120:497-512.

https://doi.org/10.1016/i.cell.2005.01.028

PMID:15734682

99. Sigurdsson S, Alexandersson KF, Sulem P, Feenstra B, Gudmundsdottir S, Halldorsson GH, Olafsson S, Sigurdsson A, Rafnar T, Thorgeirsson T, Sørensen E, Nordholm-Carstensen A, Burcharth J, et al. Sequence variants in ARHGAP15, COLQ and FAM155A associate with diverticular disease and diverticulitis. Nat Commun. 2017; 8:15789. https://doi.org/10.1038/ncomms15789 PMID:28585551
100. Pan S, Deng Y, Fu J, Zhang Y, Zhang Z, Ru X, Qin $X$. Decreased expression of ARHGAP15 promotes the development of colorectal cancer through PTEN/AKT/FOXO1 axis. Cell Death Dis. 2018; 9:673. https://doi.org/10.1038/s41419-018-0707-6 PMID:29867200

101. Bera TK, Liu XF, Yamada M, Gavrilova O, Mezey E, Tessarollo L, Anver M, Hahn Y, Lee B, Pastan I. A model for obesity and gigantism due to disruption of the Ankrd26 gene. Proc Natl Acad Sci U S A. 2008; 105:270-75.

https://doi.org/10.1073/pnas.0710978105 PMID:18162531

102. Raciti GA, Bera TK, Gavrilova O, Pastan I. Partial inactivation of Ankrd26 causes diabetes with enhanced insulin responsiveness of adipose tissue in mice. Diabetologia. 2011; 54:2911-22.

https://doi.org/10.1007/s00125-011-2263-9 PMID:21842266

103. Jin TB, Ren $Y$, Shi $X$, Jiri $M$, He N, Feng T, Yuan D, Kang L. Genetic variations in the CLNK gene and ZNF518B gene are associated with gout in casecontrol sample sets. Rheumatol Int. 2015; 35:1141-47.

https://doi.org/10.1007/s00296-015-3215-3 PMID:25591661

104. Gimeno-Valiente F, Riffo-Campos ÁL, Vallet-Sánchez A, Siscar-Lewin S, Gambardella V, Tarazona N, Cervantes A, Franco L, Castillo J, López-Rodas G. ZNF518B gene up-regulation promotes dissemination of tumour cells and is governed by epigenetic mechanisms in colorectal cancer. Sci Rep. 2019; 9:9339.

https://doi.org/10.1038/s41598-019-45411-9 PMID:31249328

105. Valiente FG, Riffo-Campos AL, Cervantes A, LópezRodas G, Franco L, Castillo J. First report of ZNF518B gene expression as a prognostic factor in colorectal cancer development: Role in tissue invasiveness. Ann Oncol. 2018; 29. https://doi.org/10.1093/annonc/mdy314.034

106. Bacos K, Gillberg L, Volkov P, Olsson AH, Hansen T, Pedersen $\mathrm{O}$, Gjesing AP, Eiberg $\mathrm{H}$, Tuomi T, Almgren P, Groop L, Eliasson L, Vaag A, et al. Blood-based biomarkers of age-associated epigenetic changes in human islets associate with insulin secretion and diabetes. Nat Commun. 2016; 7:11089. https://doi.org/10.1038/ncomms11089 PMID:27029739

107. Maier VK, Feeney CM, Taylor JE, Creech AL, Qiao JW, Szanto A, Das PP, Chevrier N, Cifuentes-Rojas C, Orkin SH, Carr SA, Jaffe JD, Mertins P, Lee JT. Functional Proteomic Analysis of Repressive 
Histone Methyltransferase Complexes Reveals ZNF518B as a G9A Regulator. Mol Cell Proteomics. 2015; 14:1435-46.

https://doi.org/10.1074/mcp.M114.044586

PMID:25680957

108. Ruiz-Martínez J, Azcona LJ, Bergareche A, MartíMassó JF, Paisán-Ruiz C. Whole-exome sequencing associates novel CSMD1 gene mutations with familial Parkinson disease. Neurol Genet. 2017; 3:e177.
https://doi.org/10.1212/NXG.0000000000000177

PMID:28808687

109. Athanasiu L, Giddaluru S, Fernandes C, Christoforou A, Reinvang I, Lundervold AJ, Nilsson LG, Kauppi K, Adolfsson R, Eriksson E, Sundet K, Djurovic S, Espeseth $\mathrm{T}$, et al. A genetic association study of CSMD1 and CSMD2 with cognitive function. Brain Behav Immun. 2017; 61:209-16.

https://doi.org/10.1016/i.bbi.2016.11.026

PMID:27890662 


\section{Supplementary Figures}

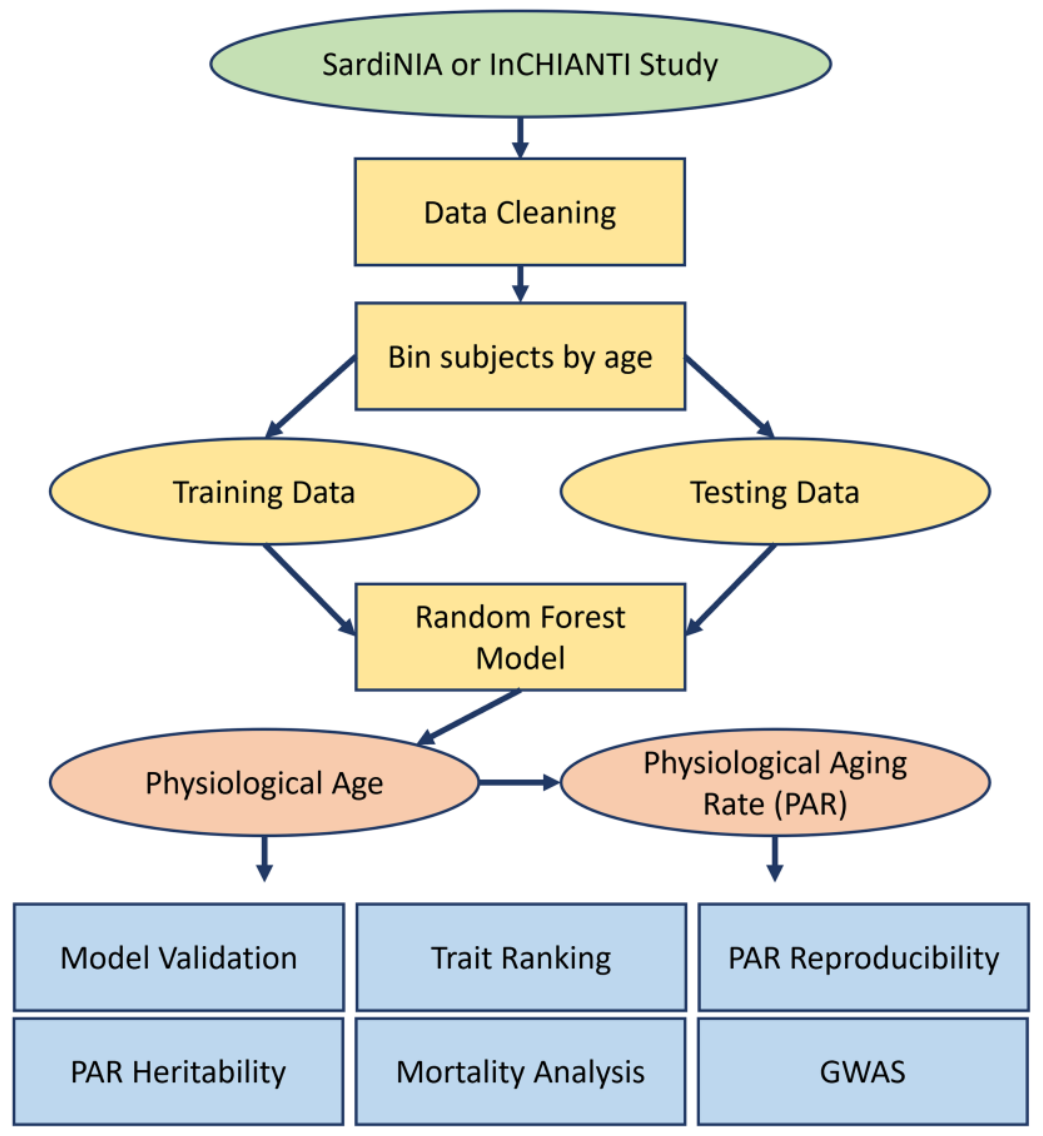

Supplementary Figure 1. Computational workflow for measuring physiological age and physiological aging rates (PAR) using the machine learning framework. These quantities were used to determine the most informative traits from the SardiNIA and InCHIANTI physiological, cognitive, and molecular markers. We measured the reproducibility and heritability of PARs. PARs from deceased participants were compared to age-matched living participants to determine the association of PAR with mortality and survival. GWAS on the PARs revealed significant genetic loci associated with differences in the aging rate. 

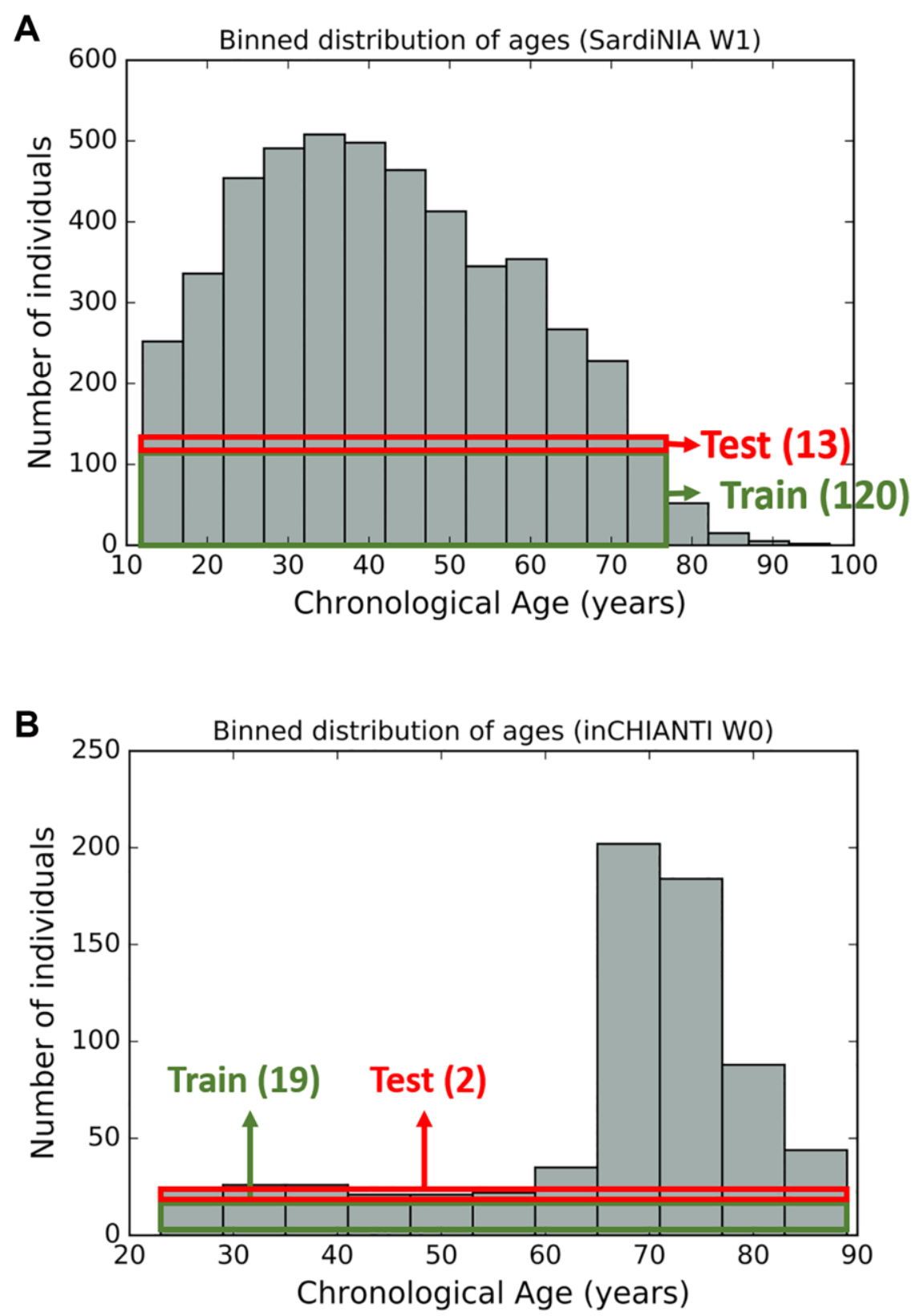

Supplementary Figure 2. Age bin distributions for the baseline SardiNIA and InCHIANTI data. Training (green) and testing (red) sample sizes per bin are labelled for the baseline study figures in each dataset. (A) In the baseline study of SardiNIA (W1), the individuals were binned into 5-year age groups spanning 12 years to 77 years of age. For each random training and testing set split, each bin contributes 120 training samples and 13 testing samples, resulting in a total set of 1560 training and 169 testing samples. (B) The baseline InCHIANTI study (WO) had 19 training and 2 testing samples per age bin (11 total bins), which resulted in a total of 209 training samples and 22 testing samples per training-testing split. Similar binning strategies for the follow-up studies and SardiNIA common-trait model are outlined in the text. 


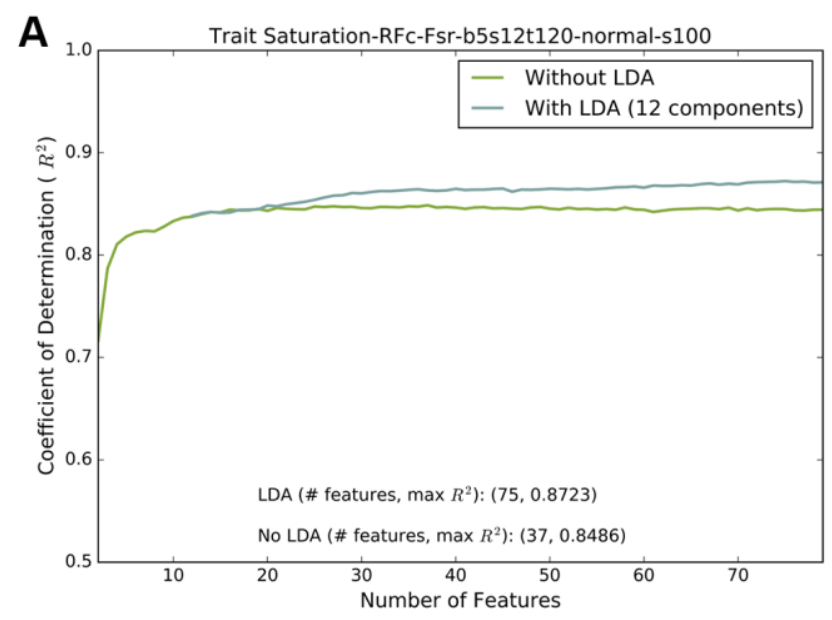

B
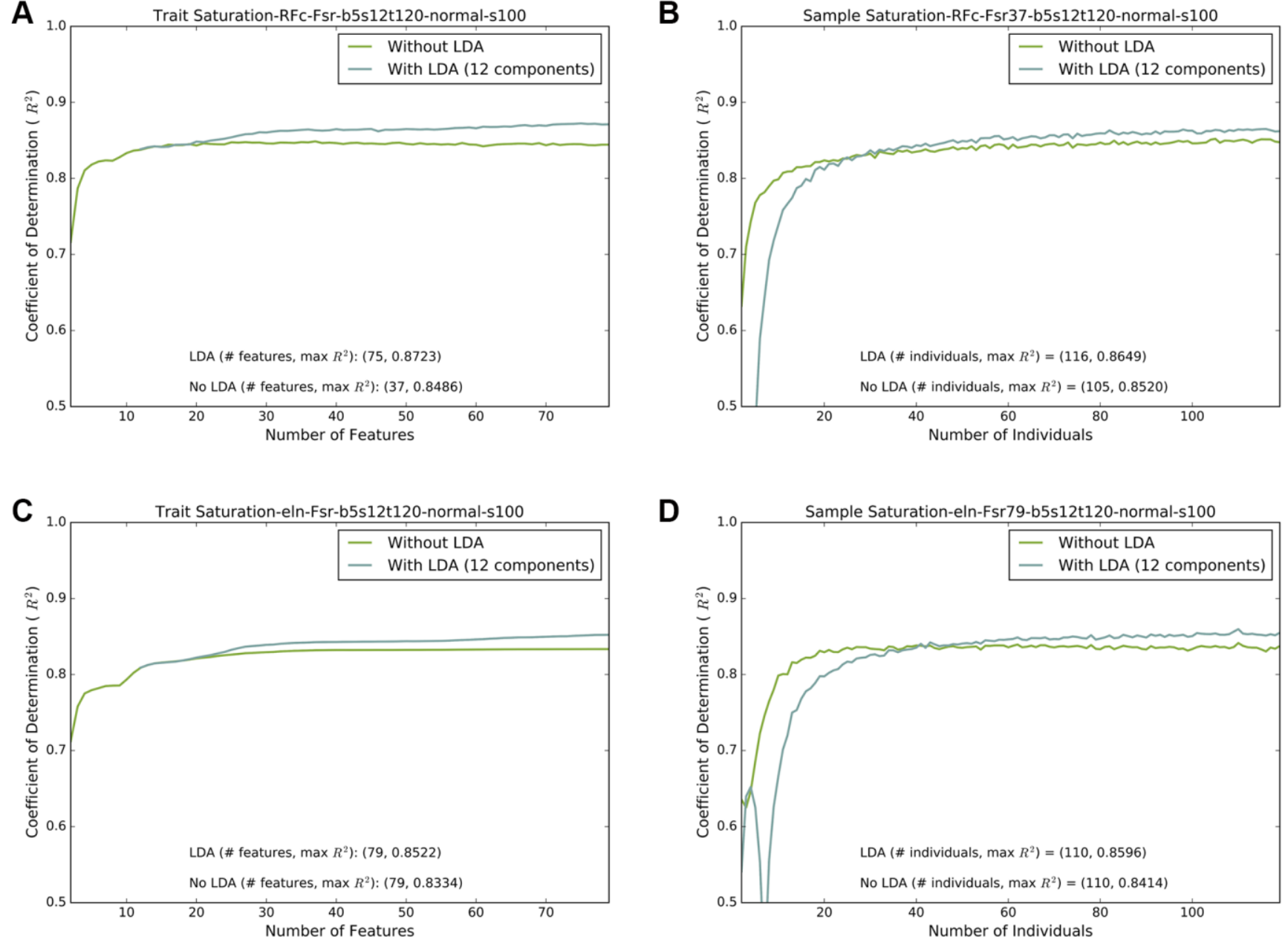

D

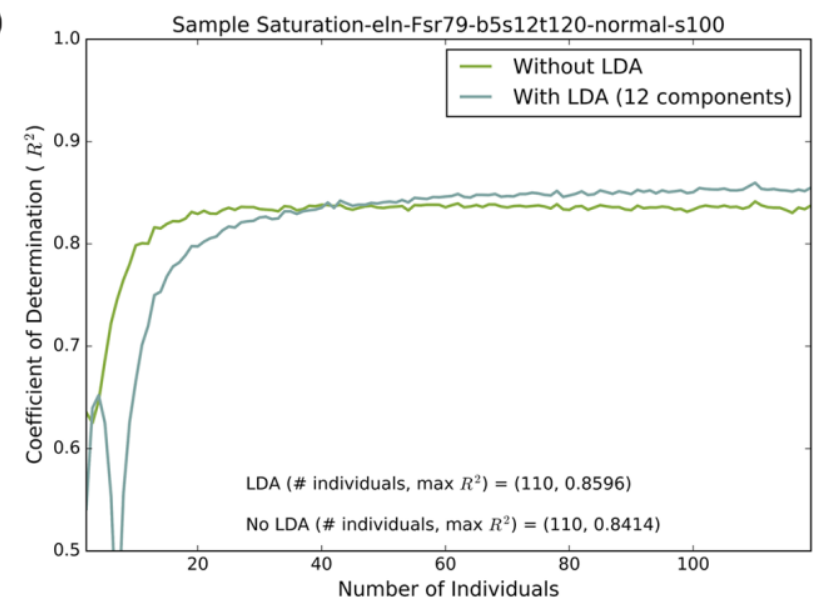

Supplementary Figure 3. Model performance saturates for increasing number of rank-ordered traits and samples. Trends for the two main machine learning models (RFC, EINet) are depicted with or without trait transformation by linear discriminant analysis (LDA). LDA improved model performance in virtually all cases. (A) Predictive performance $\left(R^{2}\right)$ of RFC saturated around 30 rank-ordered features when using all 120 individuals, and (B) saturated around 80 individuals per bin when using 37 rank-ordered features. (C) Predictive performance $\left(\mathrm{R}^{2}\right)$ of EINet saturated around 60 rank-ordered features when using all 120 individuals, and (D) saturated around 50 individuals per bin when using 79 rank-ordered features. Model performance typically saturated before the maximum number of traits or samples was reached. Results were averaged over 100 training-testing splits for the baseline SardiNIA study. 
Physiological Age


PAR
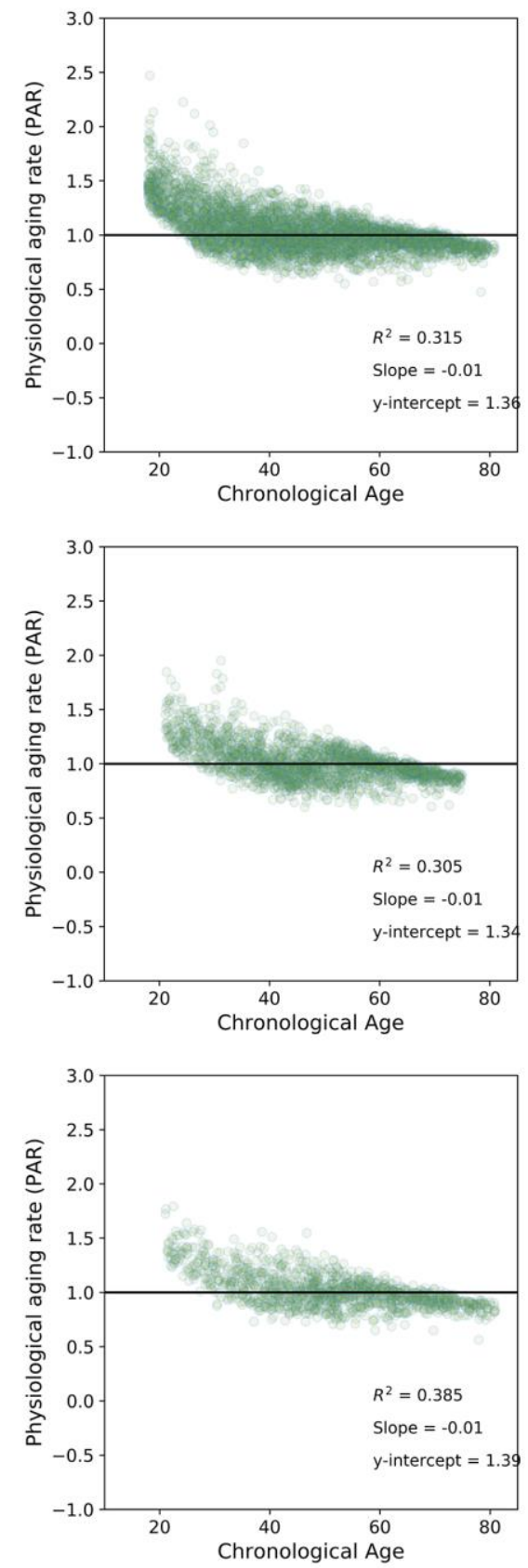

Supplementary Figure 4. Plots of physiological age and PAR against chronological age for all follow-up studies of SardiNIA using the full-trait RFC model. Physiological wages were well-correlated to chronological age while PAR measurements were weakly correlated with chronological age across all follow-up studies. Figures for the baseline SardiNIA study (W1) are shown in Figure 2C of the main text. 


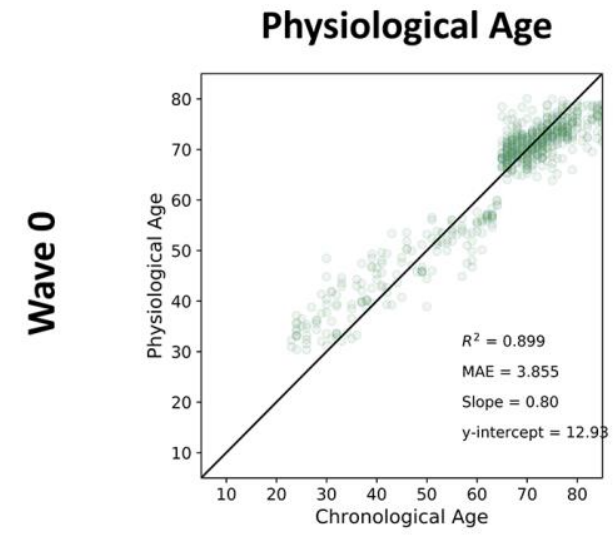

PAR
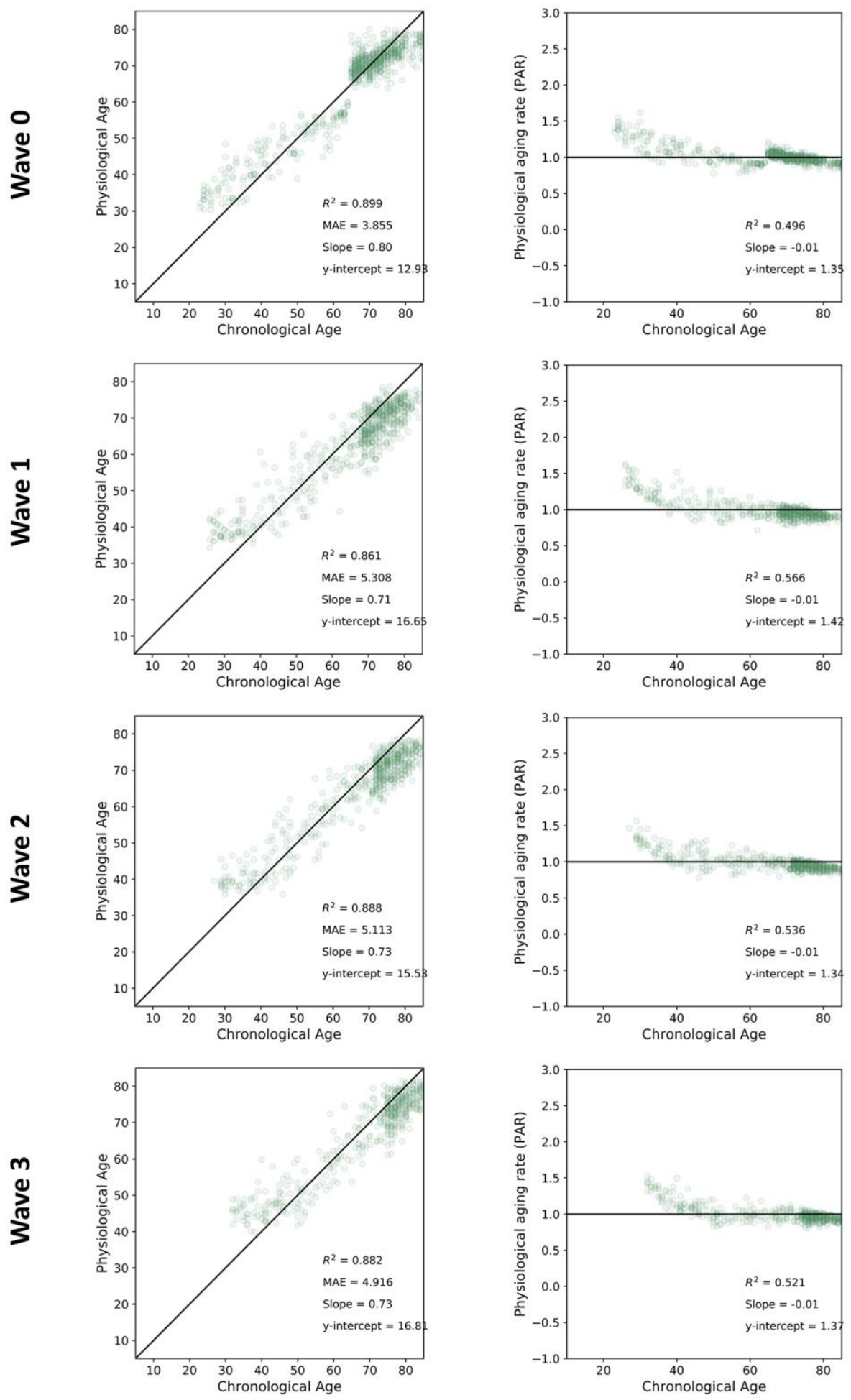

Supplementary Figure 5. Plots of physiological age and PAR against chronological age for all follow-up studies of InCHIANTI using the full-trait RFC model. Physiological wages were well-correlated to chronological age while PAR measurements were weakly correlated with chronological age across all follow-up studies. 

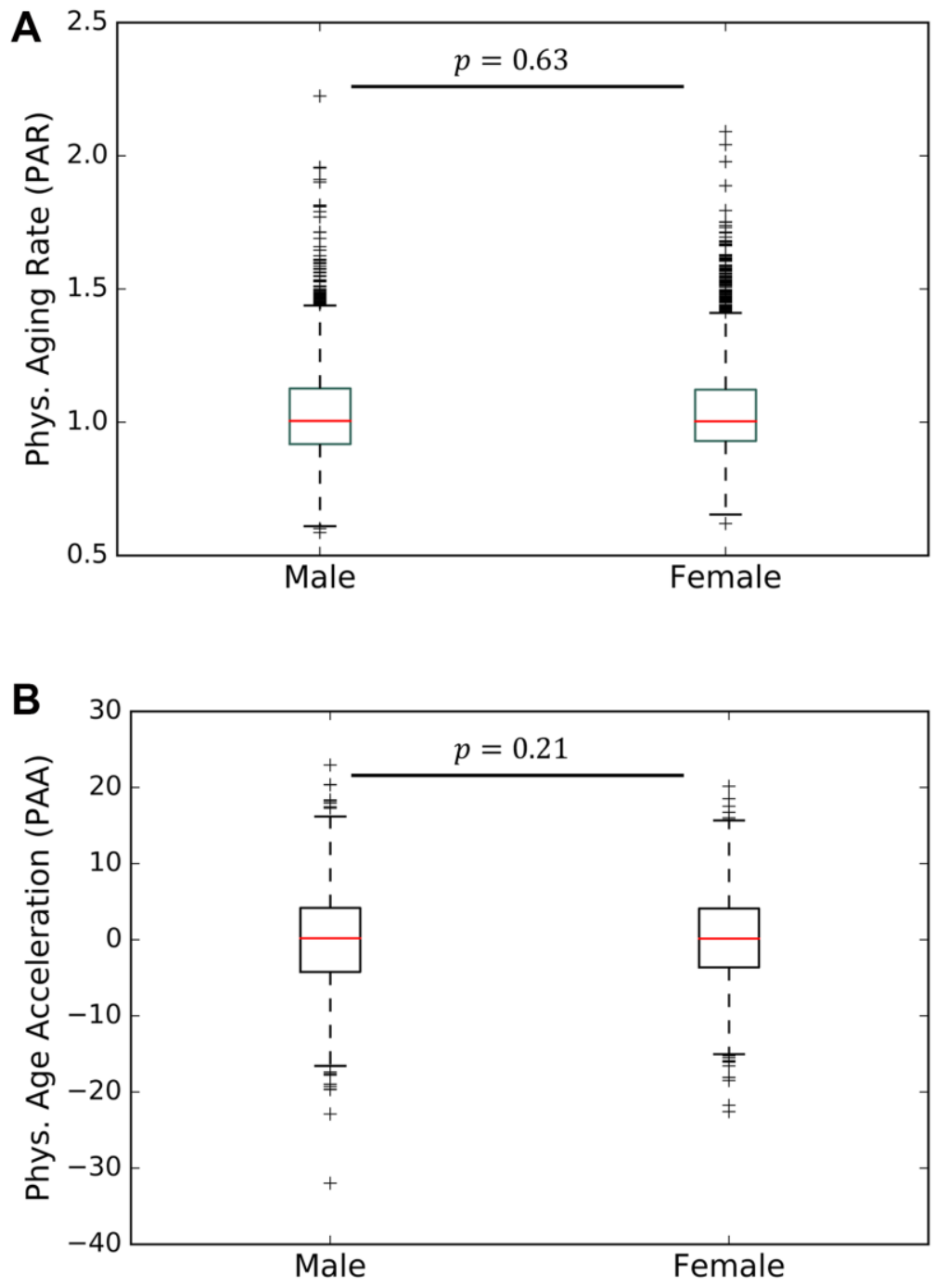

Supplementary Figure 6. Gender-separated analysis. (A) There is no significant difference between male and female participants in the physiological aging rate (PAR) obtained from the RFC model on the SardiNIA dataset. (B) Likewise, there is no significant difference in the physiological age acceleration measurements (PAA). See supplementary section on age acceleration calculation for a description of the PAA measurement. Reported $p$-values correspond to independent $t$-test of means. 
A

All Subjects

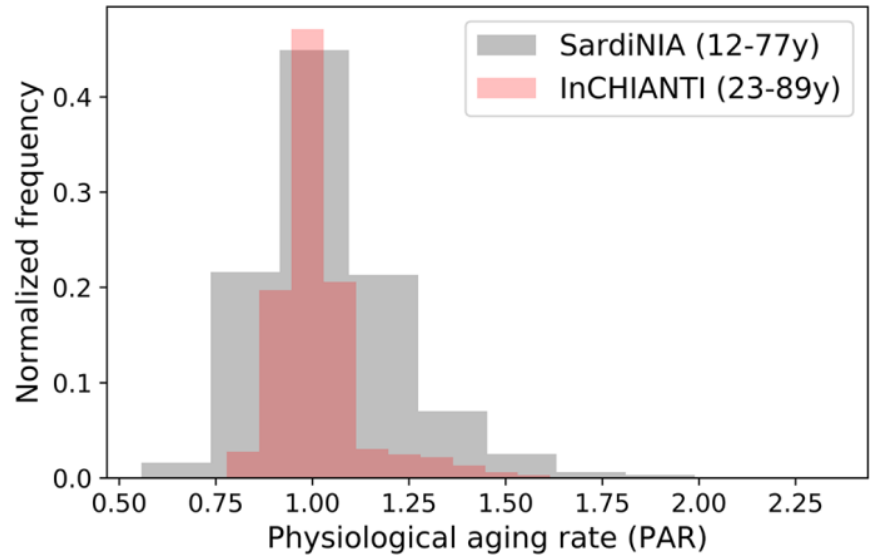

B Without Youngest and Oldest Age Bins

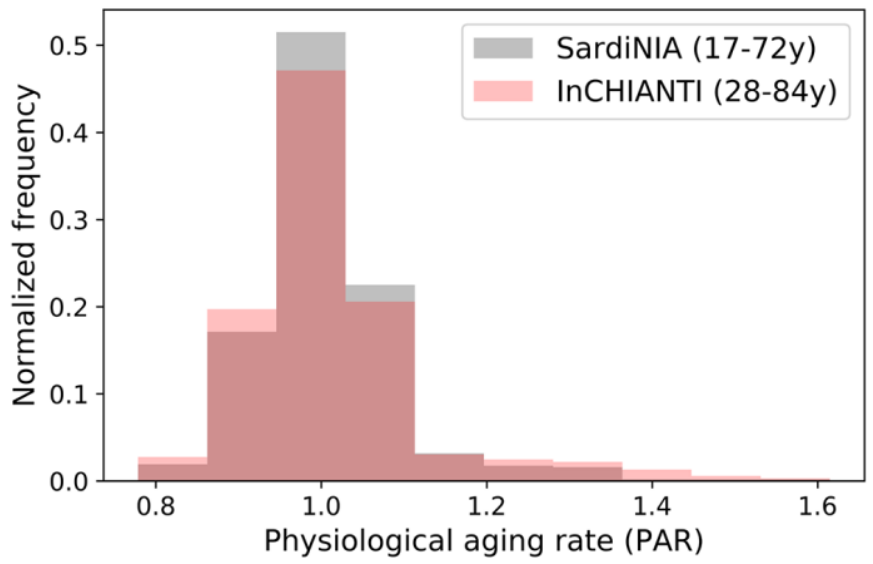

Supplementary Figure 7. Comparison of distribution of PARs in the SardiNIA (gray) and InCHIANTI (red) population studies; (A) for all individuals in each study; (B) for individuals that were not in the extreme-age (i.e. oldest and youngest) bins. 

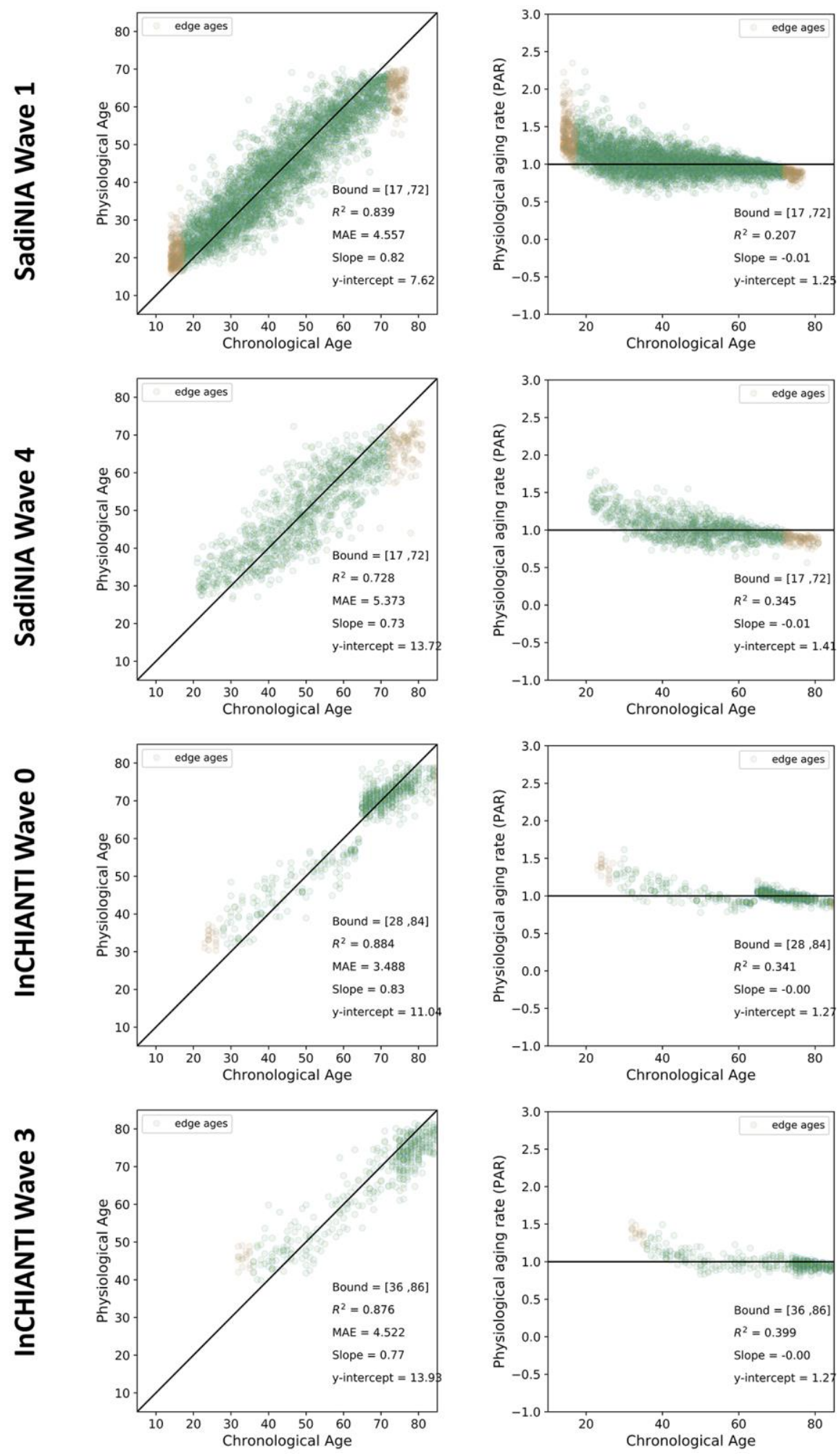

Supplementary Figure 8. Removal of youngest and oldest age bins provides more balanced PAR distribution and produces similar results. Shown are plots of physiological age and PAR against chronological age for the baseline and final follow-up studies of SardiNIA and InCHIANTI with the youngest and oldest age bins (colored brown) removed. "Bound" refers to the range of age bins that was used to compute the statistics (R2, MAE, slope and intercept of regression lines) shown in each plot. 


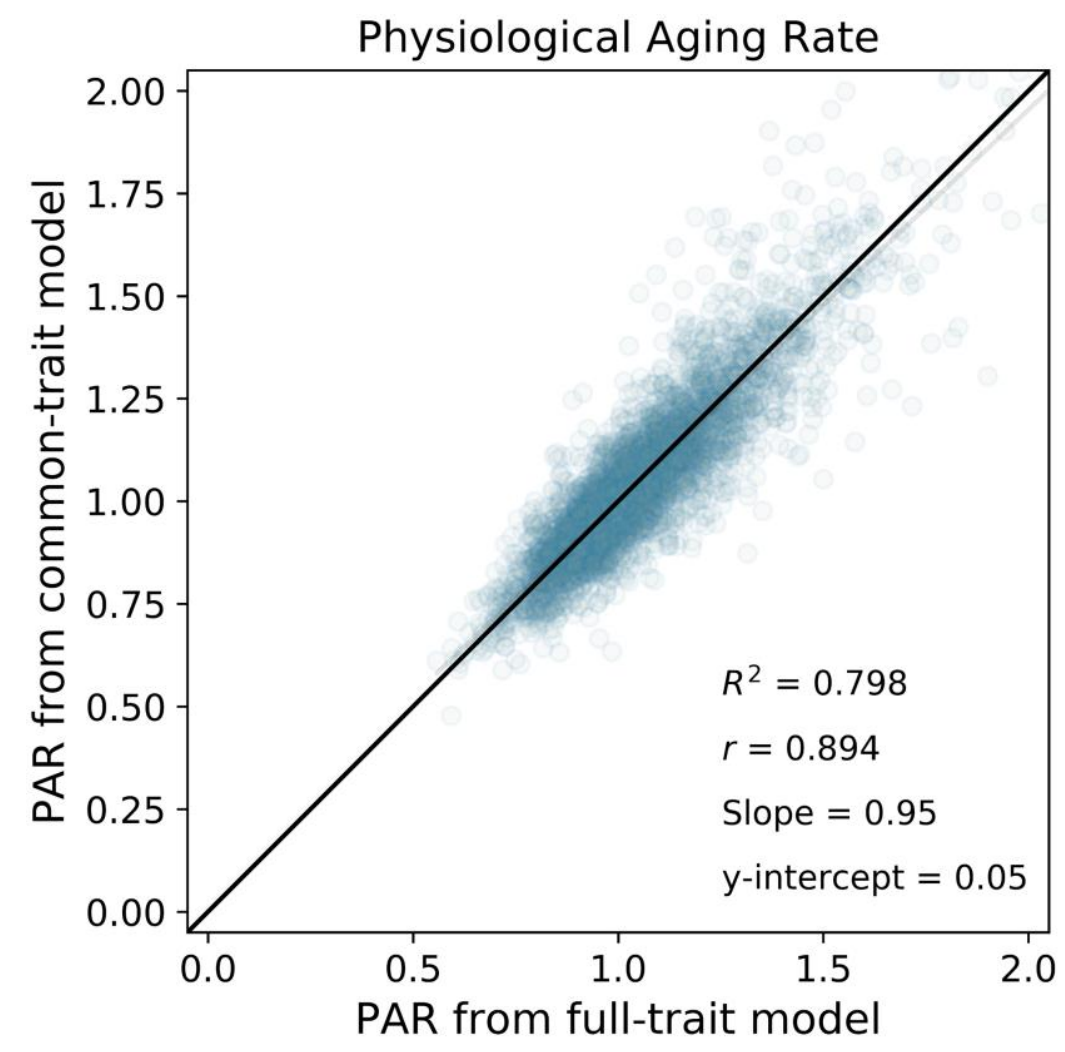

Supplementary Figure 9. Common clinical and cardiovascular traits model. Physiological aging rates (PAR) measured using the common-trait RFC model were highly correlated with the PAR measurements obtained from the full-trait RFC model. All figures were constructed using data from the baseline SardiNIA study. 

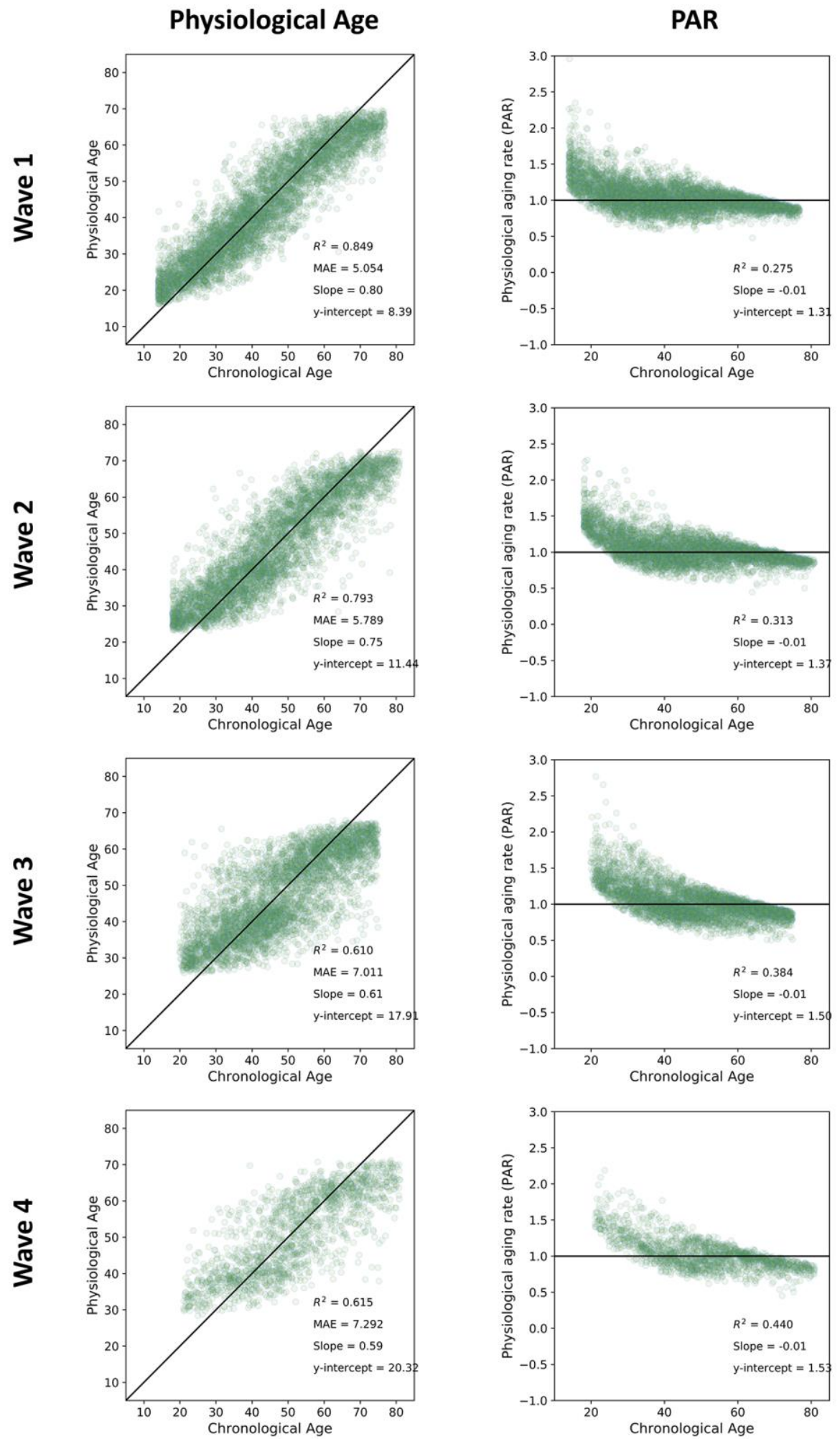

Supplementary Figure 10. Plots of physiological age and PAR against chronological age for the baseline and follow-up studies of SardiNIA using the common-trait RFC model. Results were comparable to the full-trait model. Physiological wages were well-correlated to chronological age while PAR measurements were weakly correlated with chronological age across all follow-up studies. 

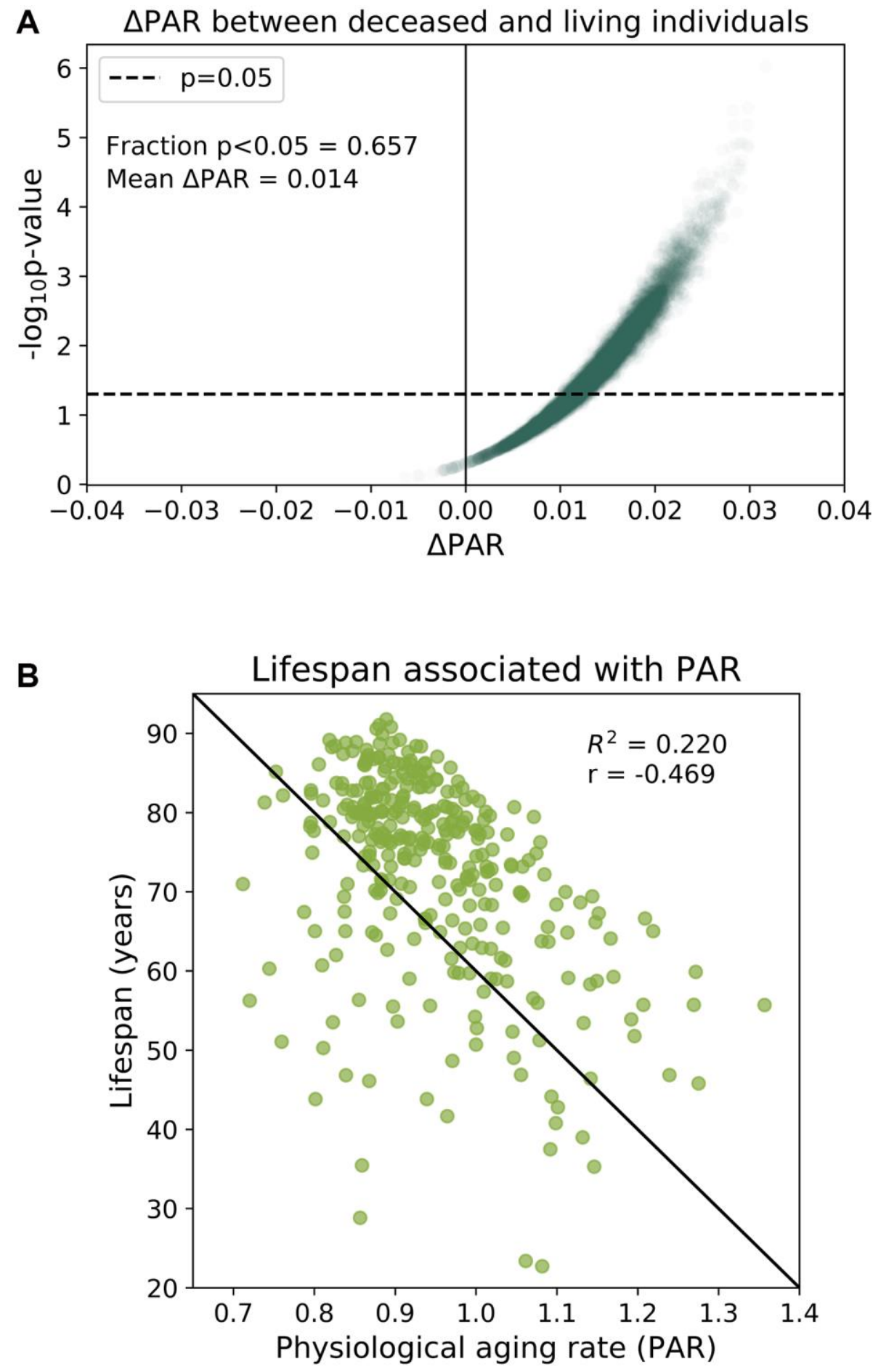

Supplementary Figure 11. Physiological aging rates from the common-trait model are associated with mortality. (A) 329 deceased participants were randomly paired with an age-matched living participant in the baseline SardiNIA study. We calculated the difference in the mean common-trait PAR measurements of the two groups, $\Delta \mathrm{PAR}=\mathrm{PAR}_{\text {deceased }}-\mathrm{PAR}_{\text {living }}$ and the corresponding $p$-value from a one-sided one-sample $t$-test for $\Delta P A R>0$. The age-matched grouping was performed 10000 times and $\Delta P A R$ and $p$-values were calculated for each of the 10000 comparisons. The fraction of significantly greater than zero $\Delta$ PAR values $(p<0.05)$ was $65.7 \%$ and the mean $\triangle P A R$ was 0.014 . Nearly all comparisons ( $>99 \%$ ) had $\Delta P A R>0$, which indicated that $P A R_{\text {deceased }}>P A R_{\text {living }}$ on average. (B) Lifespans for individuals living past 60 years were negatively correlated with PARs $(r=-0.469)$. 


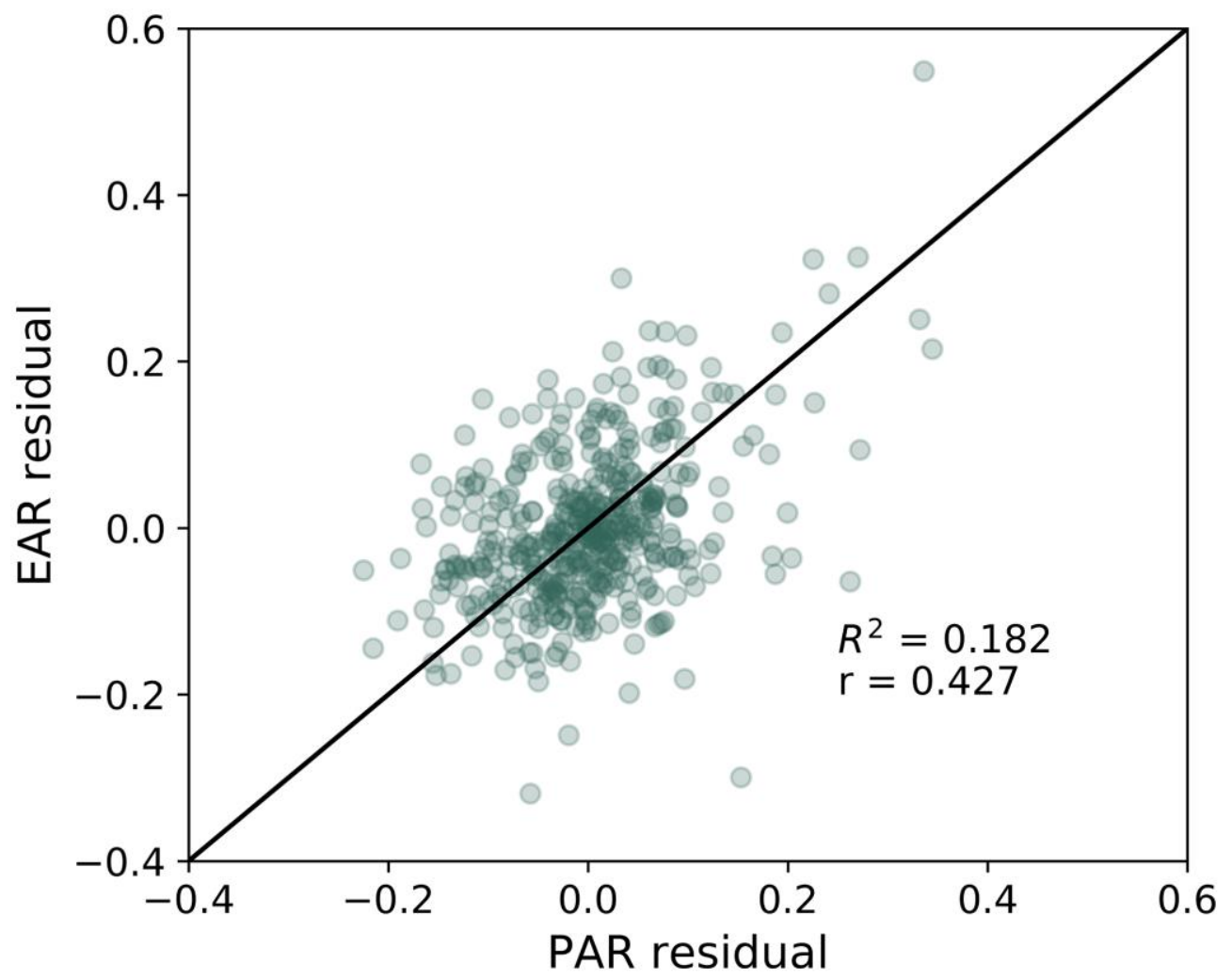

Supplementary Figure 12. Correlation between epigenetic and physiological aging rate residuals in the InCHIANTI study. Residuals $(x)$ were calculated using an ordinary least-squares regression model: EAR or PAR $=\alpha_{1} A g e+\alpha_{2}$ Sex $+x$, which served to remove the effects of chronological age and sex from the aging rates. EAR and PAR measures were still positively correlated after this adjustment.

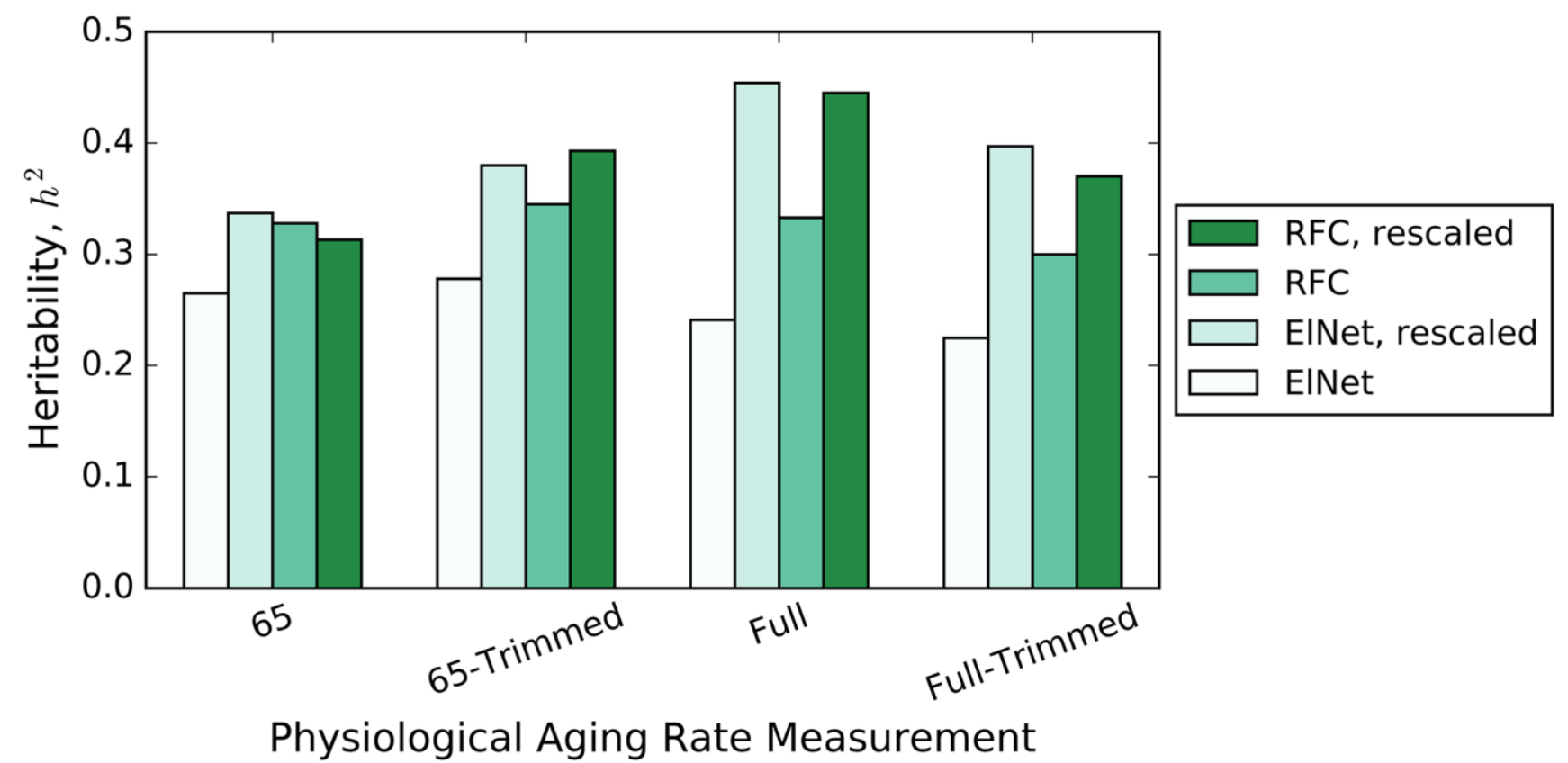

Supplementary Figure 13. Heritability scores for PARs obtained from different models on the SardiNIA data. Trimmed data refers to data where the oldest and youngest age bins were removed before model training and testing. 

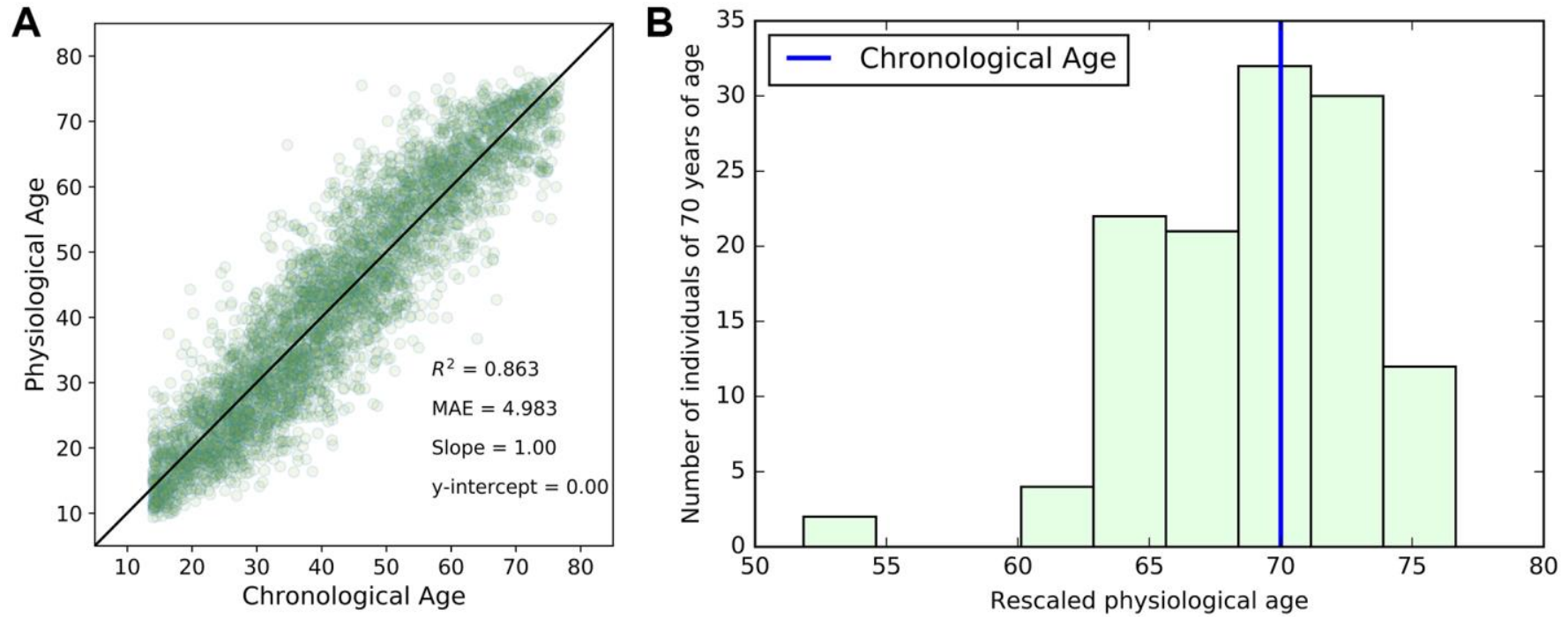

Supplementary Figure 14. Linear rescaling of physiological ages. (A) Linearly rescaling of the predicted physiological age reduced the age-dependent imbalance in PAR distributions and forced a regression slope of 1.00 between rescaled physiological age and chronological age. (B) Rescaling the physiological age preserved the assumption of approximately normally distributed physiological ages around the corresponding chronological age (blue line) for advanced ages (individual of 70 years of age are depicted). 
A Differential value in PAR quartiles

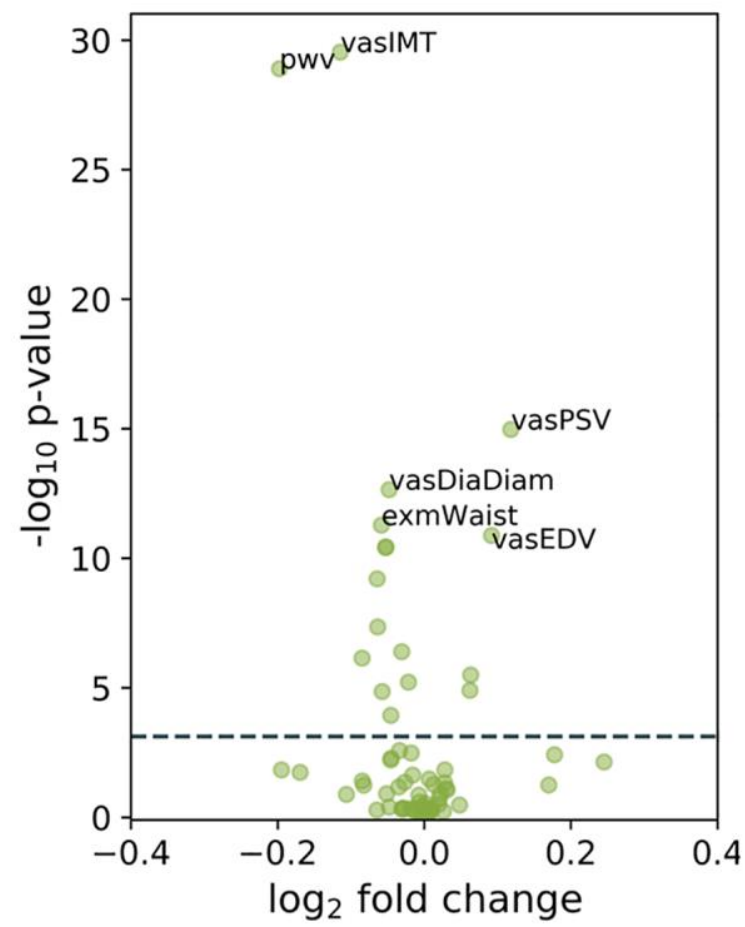

B

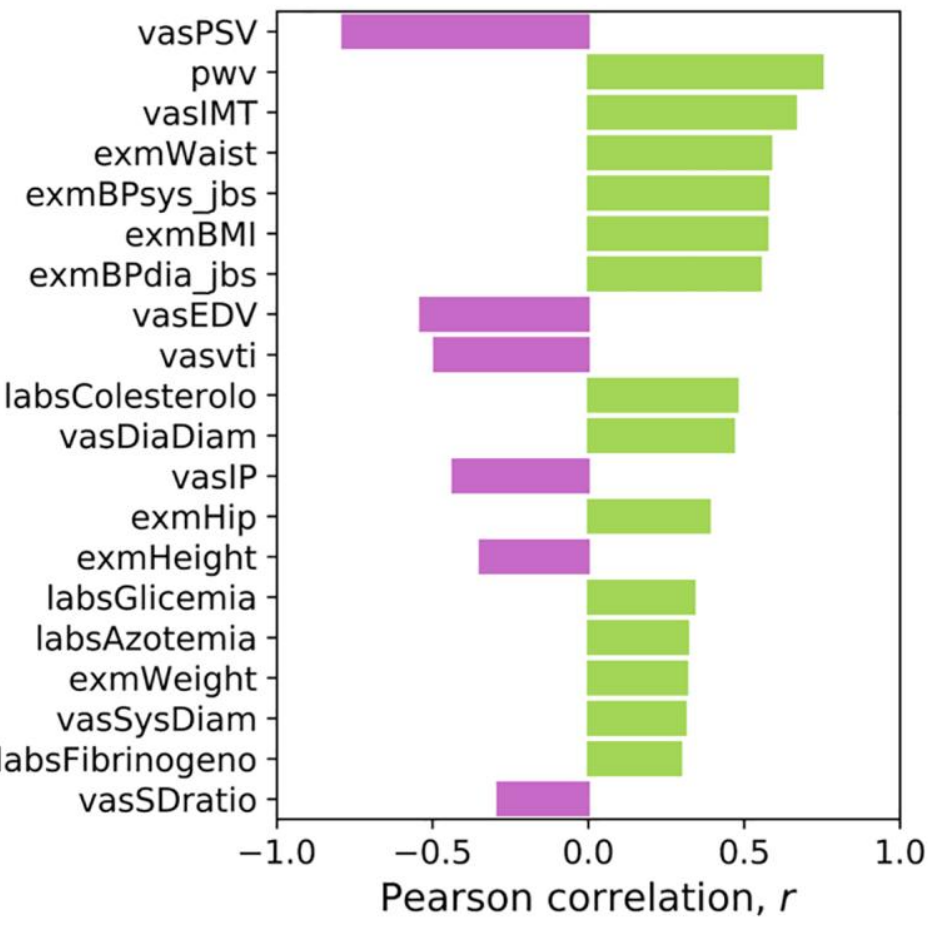

C Added value $\left(R^{2}\right)$ to $\mathrm{RFC}$ model

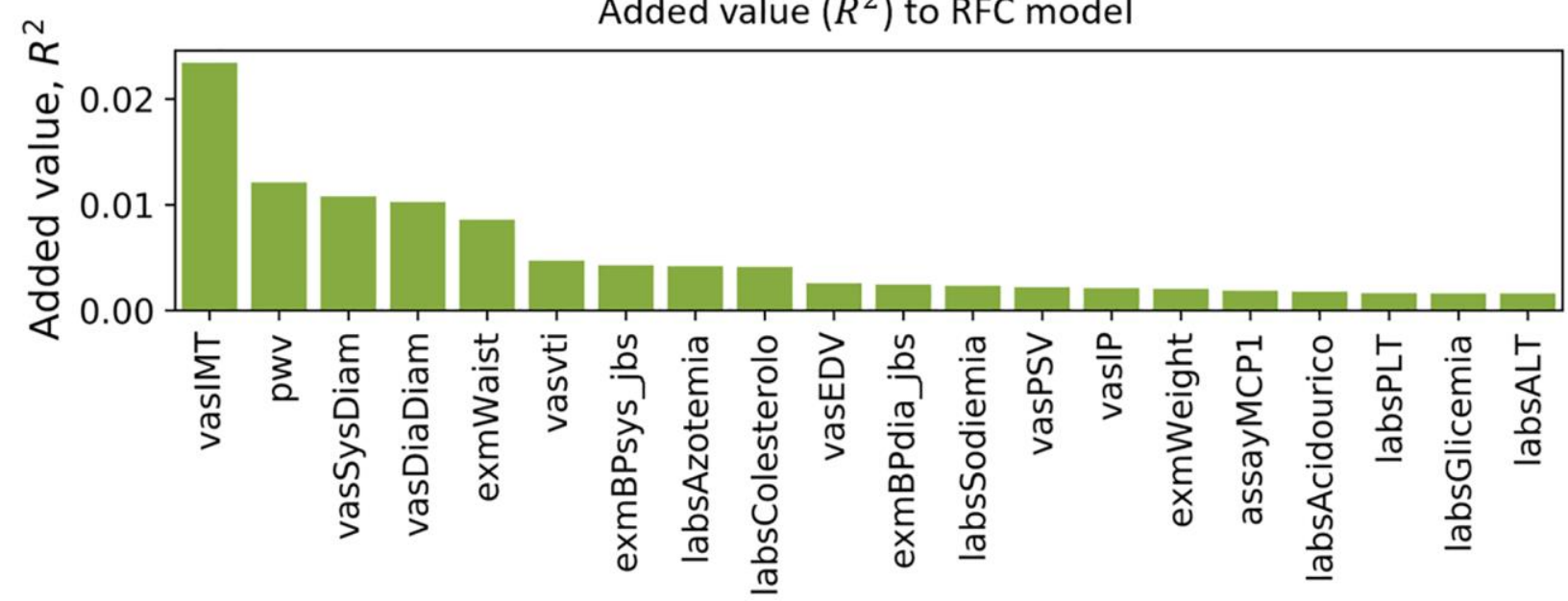

Supplementary Figure 15. Top traits from common-trait model. (A) Volcano plot of the top traits in the common-trait model included CCA intima media thickness (vasIMT), pulse wave velocity (pwv), peak systolic velocity (vasPSV), diastolic CCA diameter (vasDiaDiam), waist circumference (exmWaist), and end diastolic velocity (vasEDV). Significance was determined from a two-tailed students $t$-test on trait values from the top and bottom PAR quartiles. The dotted line corresponds to a Bonferroni-corrected $p$-value threshold of $p=$ $7.46 \times 10^{-4}$ calculated from single-test threshold of $p=5.00 \times 10^{-2}$. The top traits were very similar for the full-trait model. (B) Traits rankordered by Pearson correlation $r$ with physiological age measured using the common-trait RFC model on W1 data of SardiNIA. Top ranked traits were very similar to those from the full-trait model. (C) Traits rank-ordered by the approximate added predictive value measured using $R^{2}$ loss from $R^{2}=0.838$ for the common-trait RFC model in the baseline (W1) study of SardiNIA. $R^{2}$ added value was averaged over 500 training-testing iterates for each feature. 
A Differential value in PAR quartiles

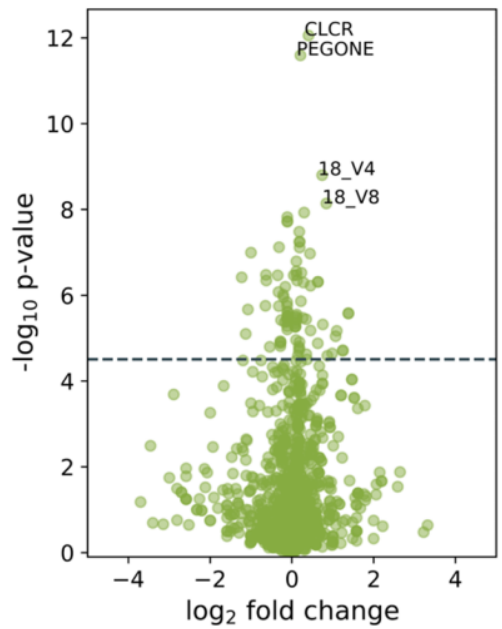

C

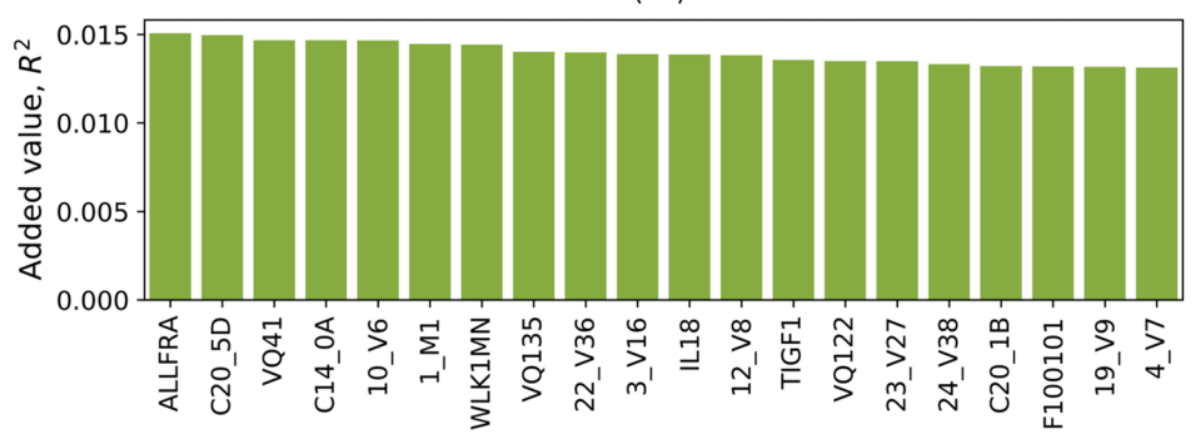

B

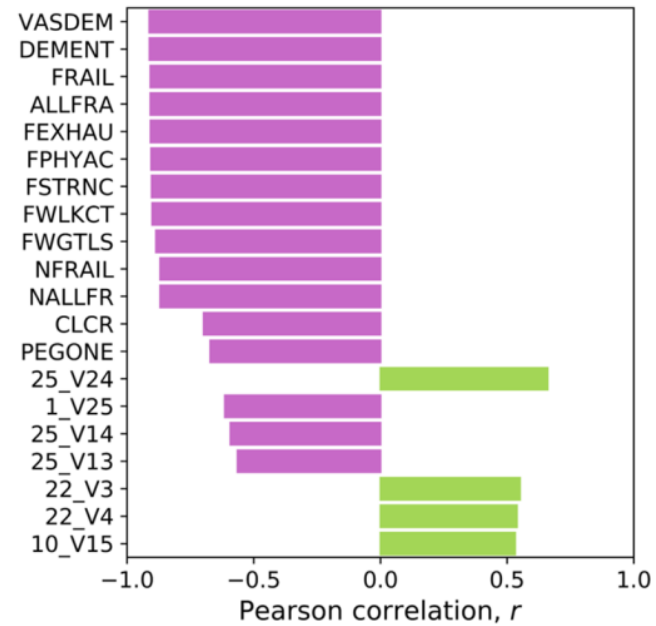

.0

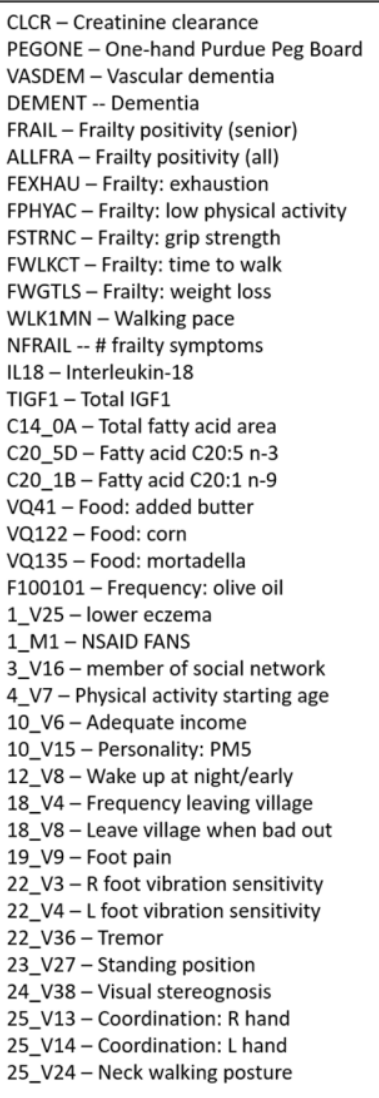

Supplementary Figure 16. Top traits from InCHIANTI. (A) Volcano plot of the top traits in the RFC model. Significance was determined from a two-tailed students $t$-test on trait values from the top and bottom PAR quartiles. The dotted line corresponds to a Bonferroni-corrected $p$-value threshold of $p=3.12 \times 10^{-5}$ calculated from single-test threshold of $p=5.00 \times 10^{-2}$. (B) Traits rank-ordered by Pearson correlation $r$ with physiological age measured using the RFC model on WO data of InCHIANTI. (C) Traits rank-ordered by the approximate added predictive value measured using $R^{2}$ loss from a baseline of $R^{2}=0.702$ for the RFC model (without feature selection) in the baseline (W0) study of InCHIANTI. $\mathrm{R}^{2}$ added value was averaged over 500 training-testing iterates for each feature. 
A Differential value in EAR quartiles

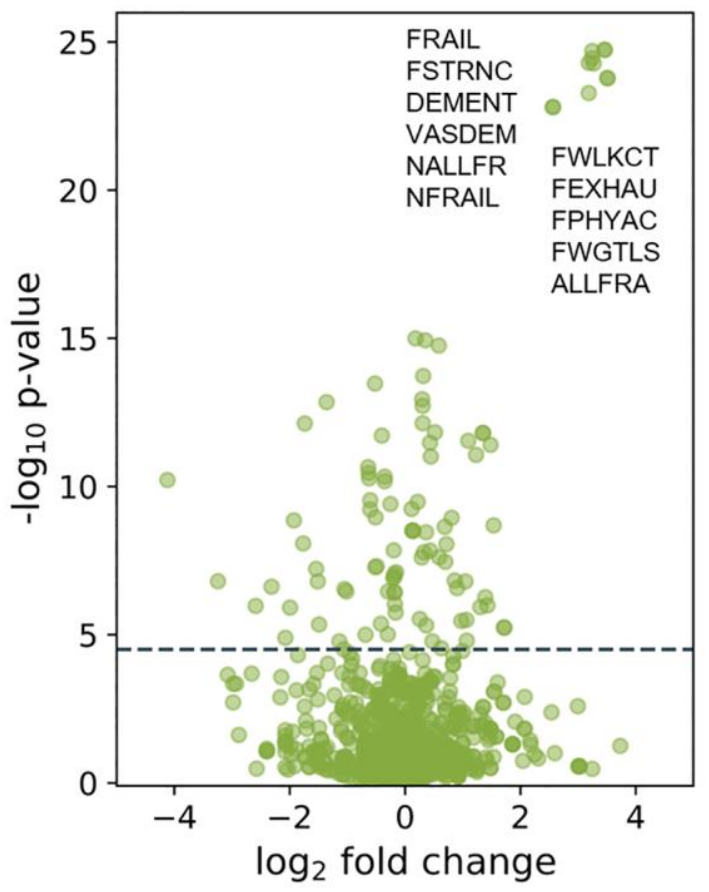

B

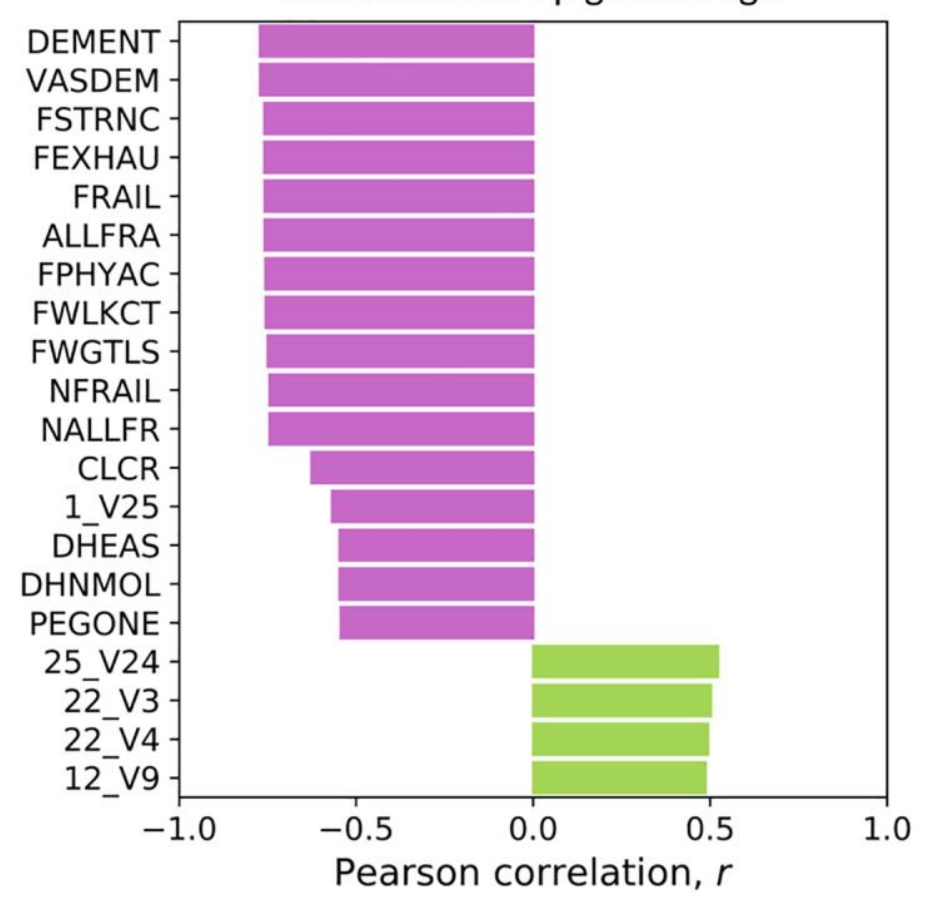

Supplementary Figure 17. Top traits according to InCHIANTI epigenetic age and EAR. (A) Volcano plot of the top traits for the epigenetic aging rate (EAR). Significance was determined from a two-tailed students $t$-test on trait values from the top and bottom EAR quartiles. The dotted line corresponds to a Bonferroni-corrected $p$-value threshold of $p=3.12 \times 10^{-5}$ calculated from single-test threshold of $p=5.00 \times 10^{-2}$. (B) Traits rank-ordered by Pearson correlation $r$ with epigenetic age measured using the Horvath model on baseline InCHIANTI data. Trait rankings were highly similar to the rankings obtained using physiological age and PAR. 

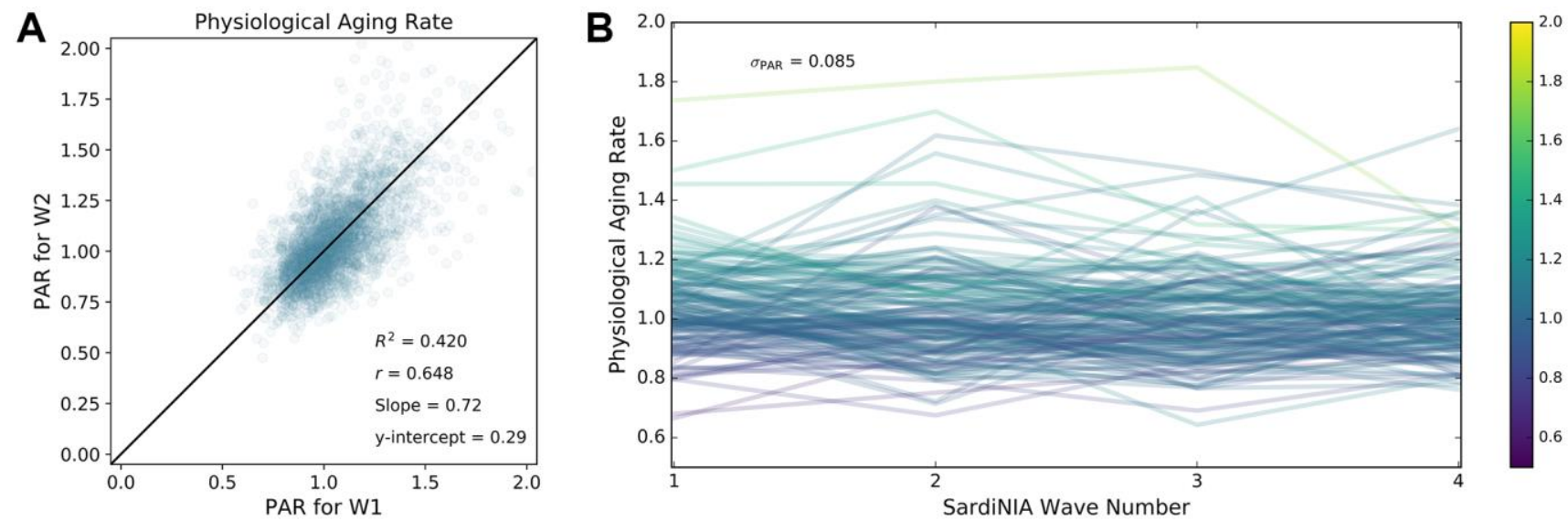

C

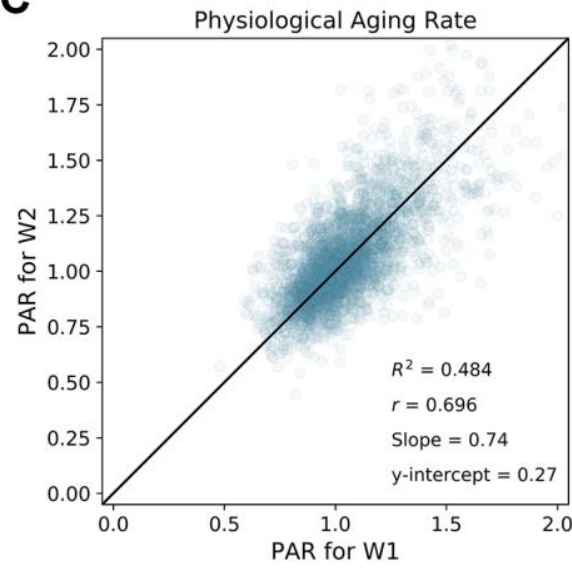

D

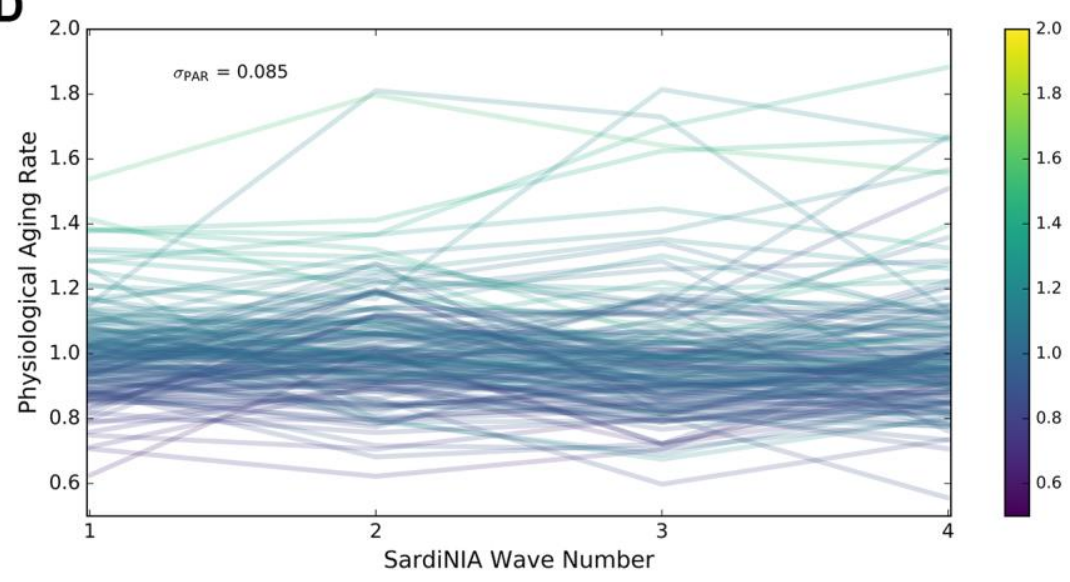

E
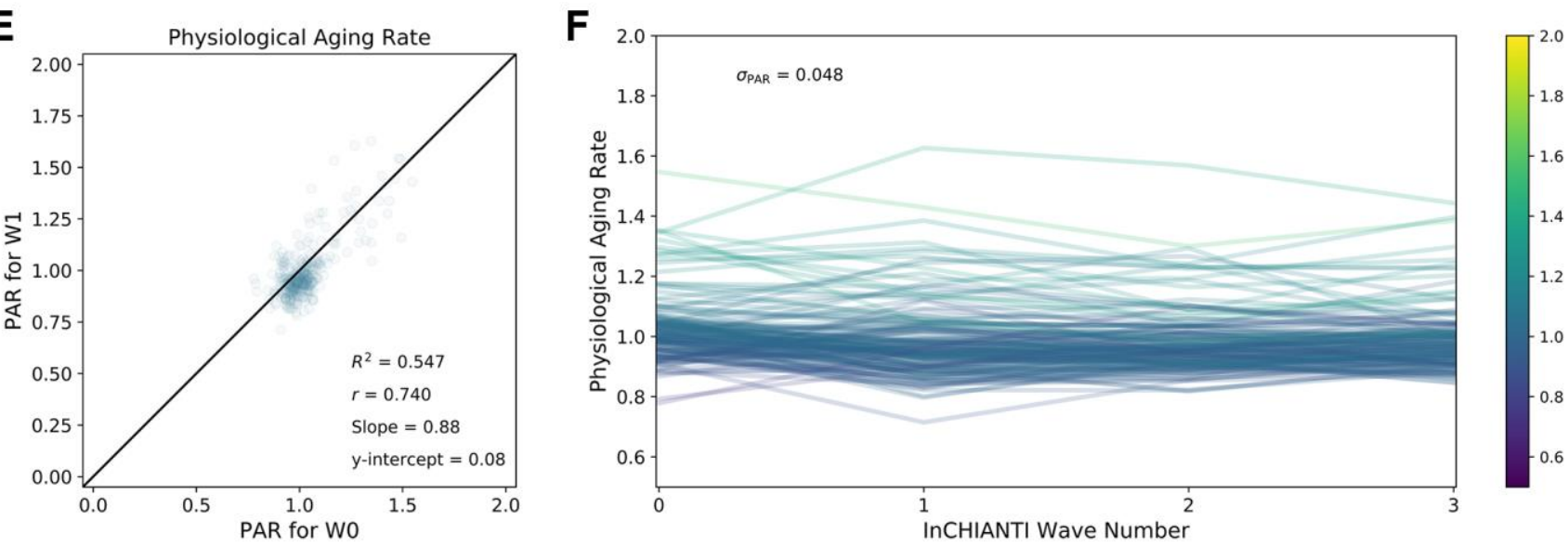

Supplementary Figure 18. Physiological aging rates (PAR) were reproducible across follow-up studies. (A) PAR values measured in the baseline (W1) study plotted against the PAR values measured in the first follow-up study (W2) of SardiNIA ( $\Delta t \approx 3$ years). PARs across W1 and W2 observed a Pearson correlation of $r=0.648$. (B) Representative plot of PAR trajectories for 150 individuals across the four SardiNIA timepoints. Trajectories colors were mapped according to an individual's baseline PAR and showcased qualitative stability for a given individual with respect to others. (C-D) Corresponding plots for the common-trait model trained on SardiNIA data. (E-F) Corresponding plots for the full-trait RFC model trained on InCHIANTI data. 
A

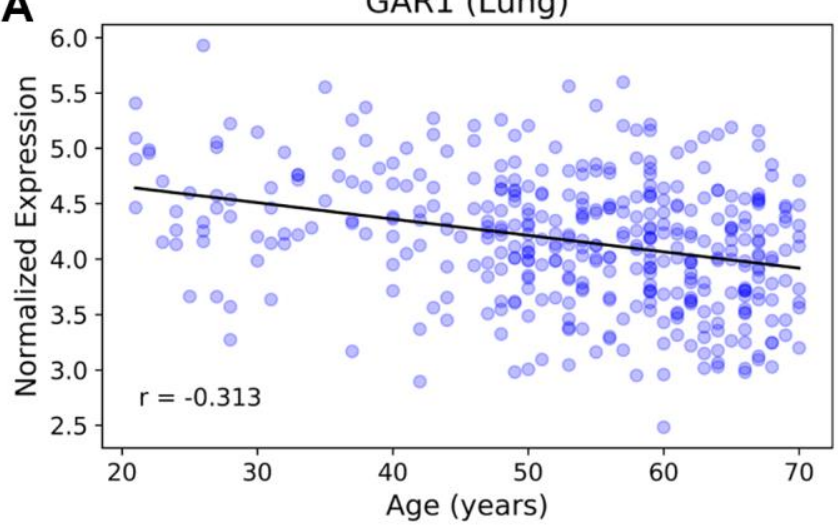

C

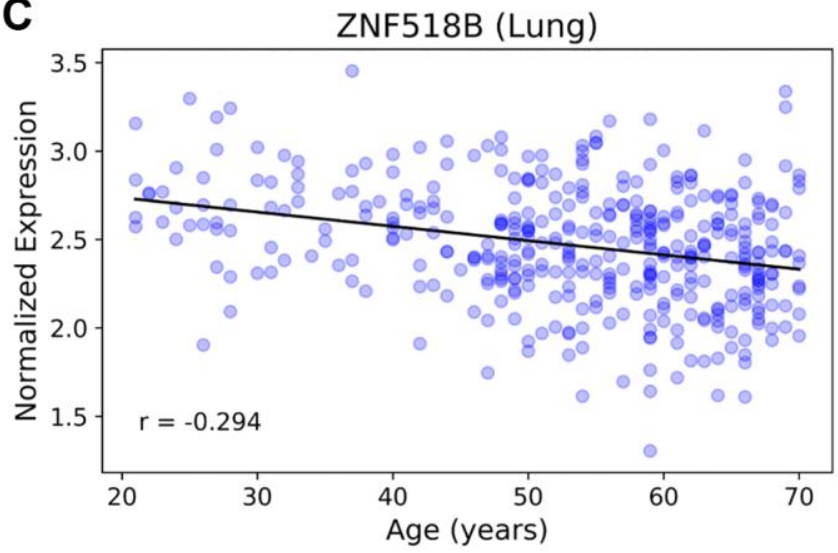

E

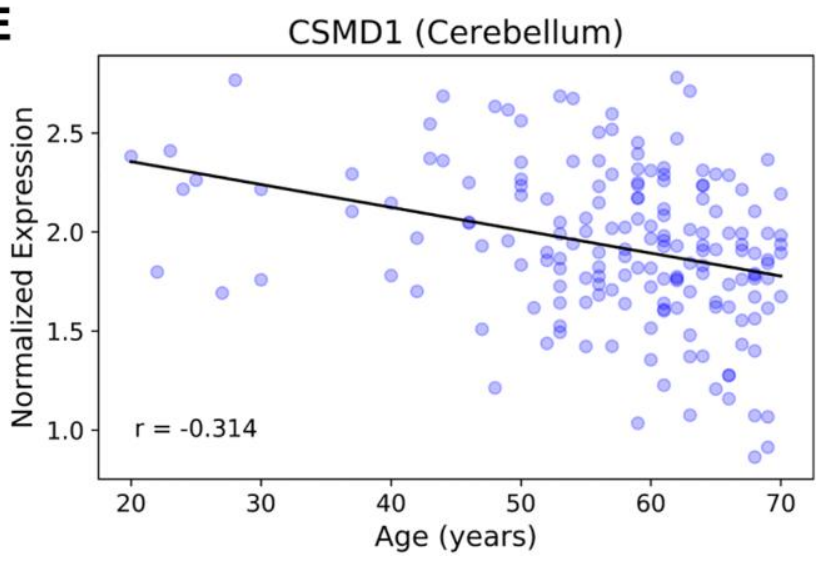

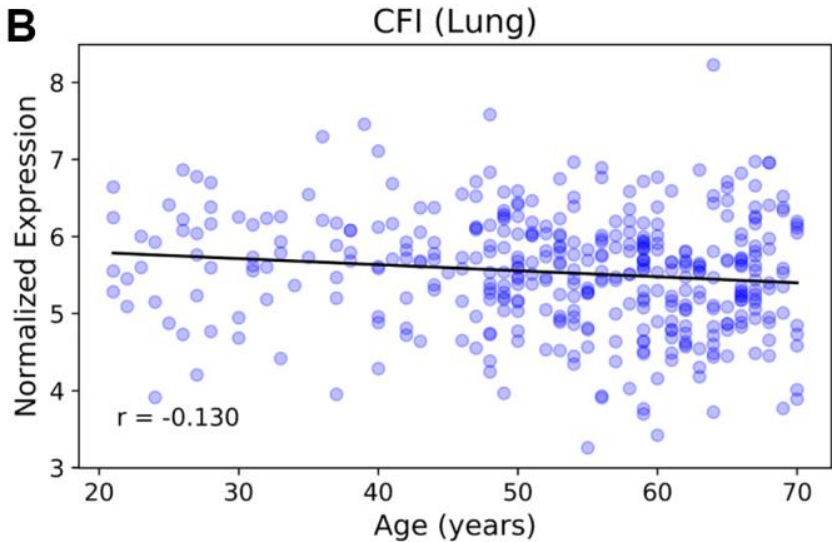

D

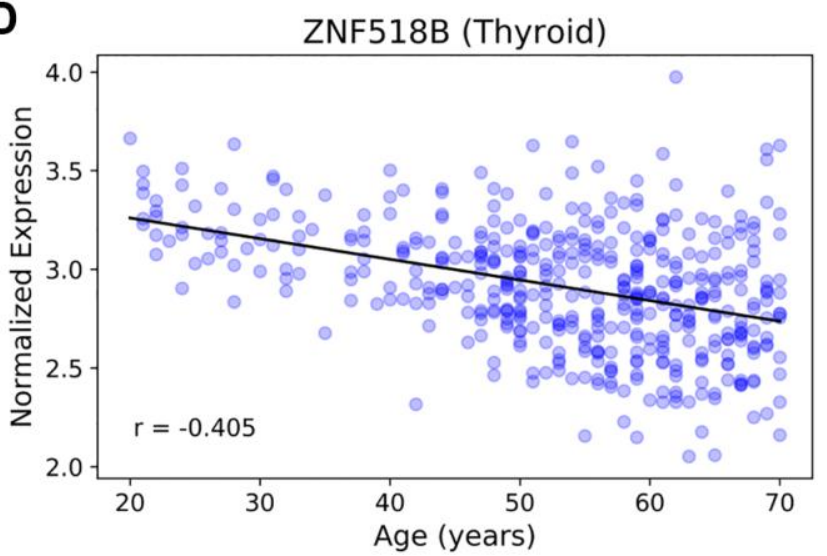

$\mathbf{F}$

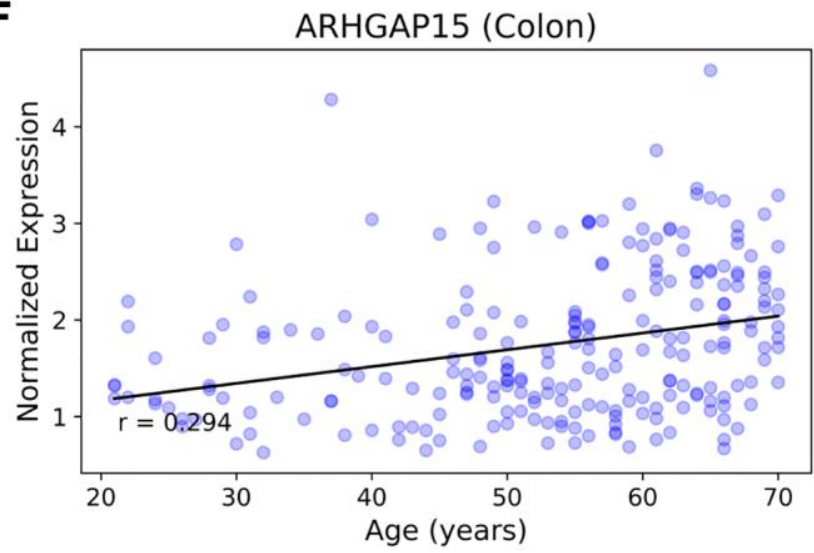

Supplementary Figure 19. Gene expression values across age in GTEx human tissues for top genome-wide significant loci. (A) Gar1 age-associated expression in GTEx lung tissue samples. (B) CFI age-associated expression in GTEx lung tissue samples. Gar1 was down-expressed in several tissues including heart (AA), lung, and colon while CFI did not appear to have strong age-dependent expression patterns in any tissues. (C) ZNF518B age-associated expression in GTEx lung tissue samples and (D) GTEx thyroid tissue samples. ZNF518B was down-expressed $(|r|>0.2)$ in the all non-brain GTEx tissues investigated: lung, thyroid, colon, heart (AA and LV), liver. (E) CSMD1 was greatly down-expressed $(r>-0.4)$ with age in the cerebellum but not in non-brain tissues, which corroborates associations between CSMD1 and neurodegenerative disease and cognitive aging. (F) ARHGAP15 was up-expressed in colon only and is associated with diverticulitis and colorectal cancer. 


\section{Supplementary Table}

Supplementary Table 1. Common clinical and cardiovascular traits included in the common-trait model for the SardiNIA study.

\begin{tabular}{|c|c|c|}
\hline Trait & Units/Measurement & Description \\
\hline labsRBC & $10^{6} / \mathrm{uL}$ & Red blood cell count \\
\hline labsHB & $\mathrm{g} / \mathrm{dL}$ & Hemoglobin lab test \\
\hline labsMCV & $\mathrm{fL}$ & Mean corpuscular volume \\
\hline labsMCH & $\mathrm{pg}$ & Mean corpuscular hemoglobin \\
\hline labsWBC & $10^{3} / \mathrm{uL}$ & White blood cell count \\
\hline labsPercNE & percentage & Neutrophil percentage \\
\hline labsPercLY & percentage & Lymphocyte percentage \\
\hline labsPercMO & percentage & Monocyte percentage \\
\hline labsPercEO & percentage & Eosinophil percentage \\
\hline labsPercBA & percentage & Basophils percentage \\
\hline \multicolumn{3}{|l|}{ V1_V5 } \\
\hline labsPLT & $10^{3} / \mathrm{uL}$ & Platelet count \\
\hline labsHBF & percentage & Fetal hemoglobin test \\
\hline labsHBA2 & percentage & Hemoglobin A2 test \\
\hline labsG6PD & $\mathrm{UI} / \mathrm{dL}$ & Glucose-6-phosphate dehydrogenase level \\
\hline labsGlicemia & $\mathrm{mg} / \mathrm{dL}$ & Glucose level \\
\hline labsInsulinemia & $\mathrm{mg} / \mathrm{dL}$ & Insulin level \\
\hline labsAzotemia & $\mathrm{mg} / \mathrm{dL}$ & Nitrogen level \\
\hline labsALT & $\mathrm{U} / \mathrm{L}$ & Alanine aminotransferase test \\
\hline labsAST & $\mathrm{U} / \mathrm{L}$ & Aspartate aminotransferase test \\
\hline labsGammaGT & $\mathrm{U} / \mathrm{L}$ & Gamma-glutamyltransferase test \\
\hline labsFibrinogeno & $\mathrm{mg} / \mathrm{dL}$ & Fibrinogen level \\
\hline labsSideremia & $\mathrm{mg} / \mathrm{dL}$ & Iron level \\
\hline labsTransferrina & $\mathrm{mg} / \mathrm{dL}$ & Transferrin level \\
\hline labsBilirubinad & $\mathrm{mg} / \mathrm{dL}$ & Fractionated bilirubin level \\
\hline labsBilirubinat & $\mathrm{mg} / \mathrm{dL}$ & Total bilirubin level \\
\hline labsAcidourico & $\mathrm{mg} / \mathrm{dL}$ & Uric acid level \\
\hline labsSodiemia & $\mathrm{mEq} / \mathrm{L}$ & Sodium level \\
\hline labsPotassiemia & $\mathrm{mEq} / \mathrm{L}$ & Potassium level \\
\hline labsVES & $\mathrm{mm} / \mathrm{h}$ & Erythrocyte sedimentation rate \\
\hline labsPCR & $\mathrm{mg} / \mathrm{dL}$ & C-reactive protein level \\
\hline labsTSH & $\mathrm{uU} / \mathrm{L}$ & Thyroid stimulating hormone level \\
\hline labsFt4 & $\mathrm{mg} / \mathrm{dL}$ & Thyroid Ft4 level \\
\hline assayAdip & $\mathrm{mg} / \mathrm{mL}$ & Adiponectin level \\
\hline assayLeptin & $\mathrm{pg} / \mathrm{mL}$ & Leptin level \\
\hline assayMCP1 & $\mathrm{pg} / \mathrm{mL}$ & Monocyte chemoattractant protein level \\
\hline assayIL6 & & Interleukin-6 level \\
\hline
\end{tabular}




\begin{tabular}{|c|c|c|}
\hline \multicolumn{3}{|l|}{ labsMCHC } \\
\hline \multicolumn{3}{|l|}{ labsHtc } \\
\hline \multicolumn{3}{|l|}{ labsMO_COUNT } \\
\hline \multicolumn{3}{|l|}{ labsEO_COUNT } \\
\hline \multicolumn{3}{|l|}{ labsBA_COUNT } \\
\hline \multicolumn{3}{|l|}{ labsLY_COUNT } \\
\hline \multicolumn{3}{|l|}{ labsNE_COUNT } \\
\hline labsCreatinina & $\mathrm{mg} / \mathrm{dL}$ & Serum creatinine level \\
\hline labsColesterolo & $\mathrm{mg} / \mathrm{dL}$ & Total cholesterol level \\
\hline labsHDL & $\mathrm{mg} / \mathrm{dL}$ & HDL cholesterol level \\
\hline labsTrigliceridi & $\mathrm{mg} / \mathrm{dL}$ & Triglycerides level \\
\hline exmHeight & $\mathrm{cm}$ & Height \\
\hline exmWeight & $\mathrm{kg}$ & Weight \\
\hline exmWaist & $\mathrm{cm}$ & Waist circumference \\
\hline exmHip & $\mathrm{cm}$ & Hip circumference \\
\hline exmBMI & $\mathrm{kg} / \mathrm{m}^{2}$ & Body mass index \\
\hline exmBPsys_jbs & & Supine blood pressure systolic \\
\hline exmBPdia_jbs & & Supine blood pressure diastolic \\
\hline pwv & $\mathrm{cm} / \mathrm{s}$ & Pulse wave velocity \\
\hline vasPSV & & Peak systolic velocity \\
\hline vasEDV & $\mathrm{mL}$ & End diastolic velocity \\
\hline vasIP & & Pulsatility index \\
\hline vasSDratio & & Systolic-diastolic ratio \\
\hline vasAT & & Acceleration time \\
\hline vasvti & & Integral time velocity \\
\hline vasSysDiam & & Systolic CCA diameter \\
\hline vasDiaDiam & & Diastolic CCA diameter \\
\hline vasIMT & & CCA intima media thickness \\
\hline
\end{tabular}

Full trait descriptions for SardiNIA and InCHIANTI are available in the Supplementary Materials. 